\title{
On existence and uniqueness for non-autonomous parabolic Cauchy problems with rough coefficients
}

\author{
Pascal Auscher* \\ Sylvie Monniaux ${ }^{\dagger}$ \\ August 12, 2018
}

Pierre Portal ${ }^{\ddagger}$

\begin{abstract}
We consider existence and uniqueness issues for the initial value problem of parabolic equations $\partial_{t} u=\operatorname{div} A \nabla u$ on the upper half space, with initial data in $L^{p}$ spaces. The coefficient matrix $A$ is assumed to be uniformly elliptic, but merely bounded measurable in space and time. For real coefficients and a single equation, this is an old topic for which a comprehensive theory is available, culminating in the work of Aronson. Much less is understood for complex coefficients or systems of equations except for the work of Lions, mainly because of the failure of maximum principles. In this paper, we come back to this topic with new methods that do not rely on maximum principles. This allows us to treat systems in this generality when $p \geq 2$, or under certain assumptions such as bounded variation in the time variable (a much weaker assumption that the usual Hölder continuity assumption) when $p<2$. We reobtain results for real coefficients, and also complement them. For instance, we obtain uniqueness for arbitrary $L^{p}$ data, $1 \leq p \leq \infty$, in the class $L^{\infty}\left(0, T ; L^{p}\left(\mathbb{R}^{n}\right)\right)$. Our approach to the existence problem relies on a careful construction of propagators for an appropriate energy space, encompassing previous constructions. Our approach to the uniqueness problem, the most novel aspect here, relies on a parabolic version of the Kenig-Pipher maximal function, used in the context of elliptic equations on non-smooth domains. We also prove comparison estimates involving conical square functions of Lusin type and prove some Fatou type results about non-tangential convergence of solutions. Recent results on maximal regularity operators in tent spaces that do not require pointwise heat kernel bounds are key tools in this study.
\end{abstract}

\section{Contents}

1 Introduction 2

1.1 Acknowledgments . . . . . . . . . . . . . . . . . . 7

2 Preliminaries 7

2.1 Function spaces . . . . . . . . . . . . . . . . . . 7

2.2 Maximal regularity operators . . . . . . . . . . . . . . . . . 11

* Laboratoire de Mathématiques d'Orsay, Univ. Paris-Sud, CNRS, Université Paris-Saclay, 91405 Orsay, France.

${ }^{\dagger}$ Aix-Marseille Université, CNRS, Centrale Marseille, I2M, UMR 7373, 13453 Marseille, France.

${ }^{\ddagger}$ Australian National University, Canberra, Australia, and Université Lille 1, France. 
$3 \quad L^{2}$-theory and energy solutions $\quad 15$

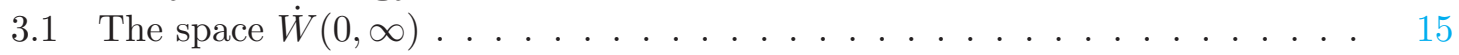

3.2 A priori energy estimates . . . . . . . . . . . . . . . . 17

3.3 Well-posedness of energy solutions . . . . . . . . . . . . . . 20

3.4 Propagators . . . . . . . . . . . . . . . . . . . . 24

3.5 Connection with earlier constructions . . . . . . . . . . . . 26

4 A priori estimates $\quad 27$

4.1 Reverse Hölder estimates and consequences . . . . . . . . . . . . . . 27

4.2 Estimates for the propagators . . . . . . . . . . . . . . . . . 29

4.3 Propagators with kernel bounds . . . . . . . . . . . . . . . . . 33

5 Existence and uniqueness results 34

5.1 Main result . . . . . . . . . . . . . . . . . . . . . 34

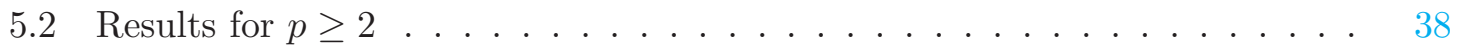

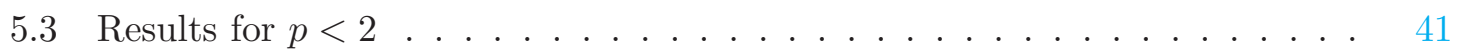

5.4 Further results . . . . . . . . . . . . . . . . . . . . 43

6 Close to constant or bounded variation time dependency 45

6.1 More about gradient bounds for semigroups . . . . . . . . . . . . . . . 45

6.2 Existence and uniqueness for $p<2$ with $B V\left(L^{\infty}\right)$ coefficients . . . . . . . 45

6.3 Existence and uniqueness for $p<2$ : small perturbations of autonomous equations or continuous coefficients . . . . . . . . . . . 48

7 Square functions and maximal functions a priori estimates 53

7.1 Controlling the maximal function by the square function for $1 \leq p<\infty$. . 53

7.2 Controlling the square function by the maximal function for $p \in[1,2) \quad \ldots \quad 54$

7.3 Controlling the square function by the maximal function for $p \in[2, \infty] \ldots 57$

7.4 Consequences . . . . . . . . . . . . . . . . . . . 61

8 Fatou type results $\quad 62$

$9 \quad L^{1}$ theory for propagators with kernel bounds 63

$\begin{array}{ll}10 \text { Local results } & 66\end{array}$

$\begin{array}{ll}\text { References } & 67\end{array}$

\section{Introduction}

We consider the problem

$$
\partial_{t} u(t, x)=\operatorname{div}(A(t, x) \nabla u(t, x)), \quad t>0, x \in \mathbb{R}^{n}
$$

where $\left.A \in L^{\infty}\left((0, \infty) \times \mathbb{R}^{n}, \mathscr{M}_{n}(\mathbb{C})\right)\right)$ satisfies uniform ellipticity estimates:

$\exists \Lambda>0$ such that $\forall \xi, \eta \in \mathbb{C}^{n},|\langle A(t, x) \xi, \eta\rangle| \leq \Lambda|\xi||\eta|$ for a.e. $t>0$ and $x \in \mathbb{R}^{n}$;

$\exists \lambda>0$ such that $\forall \xi \in \mathbb{C}^{n}, \Re e(\langle A(t, x) \xi, \xi\rangle) \geq \lambda|\xi|^{2}$ for a.e. $t>0$ and $x \in \mathbb{R}^{n}$. 
The divergence and gradient are taken with respect to the $x$ variables only. We mention right away that our results extend to systems of parabolic equations with ellipticity (1.2) replaced by a Gårding inequality on $\mathbb{R}^{n}$ uniformly with respect to $t$. For the sake of simplicity, we only consider one equation, but complex valued coefficients. We also restrict to $t>0$ since we are interested in the initial value problem with data at $t=0$. More precisely, we shall study three problems.

1) Construct weak solutions for general $L^{p}$ initial data and prove sharp estimates.

2) Show when a weak solution has a trace at $t=0$ and is uniquely determined by it.

3) Establish well-posedness as a consequence.

These problems have been studied in [30,3]; see also [28] and the references therein. Here, we obtain striking results for systems and $L^{p}$ data, as well as new results (e.g., concerning well-posedness classes) for the case of a real equation. For example, we prove uniqueness results for arbitrary $L^{p}$ data, an issue left unresolved by Aronson. Furthermore, even in the case of a real equation, our methods are technically innovative: they have to be so to circumvent the loss of maximum principles. In particular, we do not rely on the local regularity theory for solutions which culminated in [32, 31], and do not require a priori knowledge of boundedness or regularity properties of solutions in our approach.

Recall the meaning of a weak solution.

Definition 1.1. Let $0 \leq a<b \leq \infty, \Omega$ be an open subset of $\mathbb{R}^{n}$ and $Q=(a, b) \times \Omega$. A weak solution of (1.1) on $Q$ is a (complex-valued) function $u \in L_{\text {loc }}^{2}\left(a, b ; H_{\text {loc }}^{1}(\Omega)\right)$ such that

$$
\iint_{Q} u(t, x) \overline{\partial_{t} \varphi(t, x)} \mathrm{d} x \mathrm{~d} t=\iint_{Q} A(t, x) \nabla u(t, x) \cdot \overline{\nabla \varphi(t, x)} \mathrm{d} x \mathrm{~d} t
$$

for all $\varphi \in \mathscr{C}_{c}^{\infty}(Q)$. For $0 \leq a<b<\infty$ and $\Omega=\mathbb{R}^{n}$, we say that $u$ is a local (in time) solution on $(a, b)$, and when $Q=\mathbb{R}_{+}^{n+1}:=(0, \infty) \times \mathbb{R}^{n}$ we say that $u$ is a global weak solution.

Recall that well-posedness for the Cauchy problem consists in proving existence and uniqueness for global (or local) weak solutions of (1.1) $u$ in some solution space $X$, converging, as $t$ tends to 0 , to an initial data $f$ in a space of initial data $Y$, in some appropriate sense. In this case, $X$ is said to be a well-posedness class for (1.1) for $Y$ data.

This problem is well-understood for global solutions of the heat equation when $Y=$ $L^{p}\left(\mathbb{R}^{n}\right)$ and $X=L^{\infty}\left((0, \infty) ; L^{p}\left(\mathbb{R}^{n}\right)\right)$, for $p \in[1, \infty]$.

First, the heat extension of $f \in Y$ is easily seen to belong to $X$. Conversely, use of the maximum principle and form methods allow one to prove that all weak solutions (which are, in fact, classical solutions) in $X$ have a trace in $Y$ and are given by the semigroup. The most efficient arguments seem to be the ones designed for Riemannian manifolds, because they do not rely on any explicit formula for the heat kernel. Strichartz, in [35], proves this result for $1<p<\infty$, even for global solutions with $\|u(t, \cdot)\|_{L^{p}\left(\mathbb{R}^{n}\right)}$ possibly growing as $t \rightarrow \infty$ (but not faster than exponentially). For $p=1$, we refer to [29] for a neat proof, and another argument for $1<p<\infty$. For $p=\infty$, see [18] for a uniqueness result under a continuity assumption.

Back to the Euclidean case for the non-autonomous problem (1.1), it was Aronson [3] who obtained the most complete results for real equations in divergence form. He considers the energy space $L^{\infty}\left(0, T ; L^{2}\left(\mathbb{R}^{n}\right)\right) \cap L^{2}\left(0, T ; H^{1}\left(\mathbb{R}^{n}\right)\right)$. He proves that all solutions $u$ in this space have a trace $u_{0}$ in $L^{2}\left(\mathbb{R}^{n}\right)$, and are uniquely determined by this trace. It follows that this class is a uniqueness class. Aronson also obtains existence given an $L^{2}$ initial data, 
hence defines a propagator $\Gamma$ such that $u(t, \cdot)=\Gamma(t, 0) u_{0}$ for $t>0$. The same strategy, with a slightly different energy space, was employed by Lions [30] earlier for complex equations, and it yields the same solution. For real equations, however, Aronson also proved pointwise Gaussian decay of the propagator in [2]. This allows one to define weak solutions by the integral representation

$$
u(t, x)=\int_{\mathbb{R}^{n}} k(t, 0, x, y) u_{0}(y) \mathrm{d} y
$$

for $u_{0}$ in various spaces of initial conditions. For solutions satisfying an integral condition

$$
\|u\|_{\mathcal{E}}^{2}:=\int_{0}^{T} \int_{\mathbb{R}^{n}} e^{-a|x|^{2}} u(t, x)^{2} \mathrm{~d} t \mathrm{~d} x<\infty
$$

for some $a>0$, Aronson proves uniqueness in this class, and existence given $u_{0} \in L^{2}\left(e^{-\gamma|x|^{2}} \mathrm{~d} x\right)$ (with an assumption linking $\gamma>0, T>0$ and $a$ ). This covers $u_{0} \in L^{p}(\mathrm{~d} x)$ with $2 \leq p \leq \infty$, but note that $\|u\|_{\mathcal{E}}$ is not comparable to $\left\|u_{0}\right\|_{L^{p}}$. Aronson's class may thus not be optimal for uniqueness (one could look for a larger one). We are not mentioning here the results for non-negative solutions as they are clearly outside the scope of the present article, since we want to address complex equations.

Let us come back to the heat equation and consider solutions given by $u(t, x)=e^{t \Delta} f(x)$ for, say, $f \in L^{2}$. In harmonic analysis, there are other well-known estimates for such solutions given in terms of the non-tangential maximal function and the Lusin area functionals:

$$
\left\|u^{*}\right\|_{L^{p}} \sim\|\nabla u\|_{T^{p, 2}}, \quad 1 \leq p<\infty .
$$

Here $T^{p, 2}$ denotes the tent space of Coifman-Meyer-Stein. See Section 2.1 for its definition. We denote by $u^{*}$ the non-tangential maximal function $x \mapsto \sup _{|y-x|<\sqrt{t}}|u(t, y)|$. A key feature of these estimates is that they hold also for some $p \leq 1$, and play a fundamental role in Hardy space theory.

For example, $\left\|u^{*}\right\|_{L^{p}}<\infty$ characterises the real Hardy space $H^{p}$ as shown in [20]. When $1<p<\infty$, an implicit argument (it is done for harmonic functions but the same idea applies to caloric functions) in [20], using Fatou type results (based on the maximum principle), shows that all weak solutions of the heat equation satisfying $\left\|u^{*}\right\|_{L^{p}}<\infty$ are given by the semigroup, and thus are uniquely determined by their traces in $L^{p}$ at $t=0$. As we have comparability of norms, uniqueness in such a class is an optimal result for $L^{p}$ data. It is not known to us whether the condition $\|\nabla u\|_{T^{p, 2}}<\infty$ with $u$ vanishing at $\infty$ yields uniqueness (recall that $\nabla$ is only with respect to $x$ ) except when $p=2$.

Our approach to (1.1) starts as in Aronson ([3]) or Lions ([30]), by considering energy solutions. If $u$ is either one's solution (it turns out that they are the same) for a data $u_{0} \in L^{2}\left(\mathbb{R}^{n}\right)$, one obtains the energy equality

$$
\left\|u_{0}\right\|_{L^{2}}^{2}=2 \Re e \int_{0}^{T} \int_{\mathbb{R}^{n}} A(s, x) \nabla u(s, x) \cdot \overline{\nabla u(s, x)} \mathrm{d} s \mathrm{~d} x+\|u(T)\|_{L^{2}}^{2} .
$$

By taking the limit as $T \rightarrow \infty$, provided that $u$ is a global weak solution and that $\|u(T)\|_{L^{2}} \rightarrow 0$, one obtains

$$
\left\|u_{0}\right\|_{L^{2}}^{2}=2 \Re e \int_{0}^{\infty} \int_{\mathbb{R}^{n}} A(s, x) \nabla u(s, x) \cdot \overline{\nabla u(s, x)} \mathrm{d} s \mathrm{~d} x .
$$


This equality suggests that it should be possible to work directly in the largest possible energy space to begin with, consisting of global weak solutions with $\nabla u \in L^{2}\left(\mathbb{R}_{+}^{n+1}\right)=L^{2}\left(L^{2}\right)$. We prove that this is indeed the case, and establish well-posedness of the Cauchy problem for $L^{2}\left(\mathbb{R}^{n}\right)$ data in this energy space. We also show that such solutions are continuous from $[0, \infty)$ into $L^{2}\left(\mathbb{R}^{n}\right)$, norm decreasing in time with limit 0 at $\infty$, and satisfying this energy equality, of course, together with

$$
\left\|u_{0}\right\|_{L^{2}}=\|u\|_{L^{\infty}\left(L^{2}\right)} \sim\|\nabla u\|_{L^{2}\left(L^{2}\right)} .
$$

This is to be expected but note that the lack of a priori control on the $L^{2}\left(\mathbb{R}^{n}\right)$ norm in our energy space is a difficulty which we overcome thanks to a structural lemma for this space. As a consequence, we recover, by restriction to finite intervals $(0, T)$, the Aronson/Lions solutions. This gives rise to a propagator $\Gamma(t, s)$ that sends a data at time $s$ to the solution at time $t$. The only available estimates for this propagator in full generality are Gaffney type estimates, which are localized $L^{2}$ Gaussian bounds. The same holds for the backward in time adjoint equation. Using properties of this adjoint propagator to create test functions for (1.1), our main result towards uniqueness is an interior reproducing formula for local weak solutions under a certain control.

Theorem 1.2. Let $u$ be a local weak solution of $(1.1)$ on $(a, b) \times \mathbb{R}^{n}$. Assume

$$
M:=\int_{\mathbb{R}^{n}}\left(\int_{a}^{b} \int_{B(x, \sqrt{b})}|u(t, y)|^{2} \mathrm{~d} y \mathrm{~d} t\right)^{\frac{1}{2}} e^{-\gamma|x|^{2}} \mathrm{~d} x<\infty
$$

for some $0<\gamma<\gamma(a, b, \lambda, \Lambda)$. Then $u(t, \cdot)=\Gamma(t, s) u(s, \cdot)$ for every $a<s \leq t<b$, in the following sense:

$$
\int_{\mathbb{R}^{n}} u(s, x) \overline{\Gamma(t, s)^{*} h(x)} \mathrm{d} x=\int_{\mathbb{R}^{n}} u(t, x) \overline{h(x)} \mathrm{d} x \quad \forall h \in \mathscr{C}_{c}\left(\mathbb{R}^{n}\right) .
$$

Note that the control is in terms of local $L^{2}$ estimates on $u$. This is the only available information. Also the presence of the square root in the control turns out to be very useful.

Once this is proved the matter reduces to controlling $u$ near the boundary $t=0$ to be able to take a limit as $s$ tends to 0 in $u(t, \cdot)=\Gamma(t, s) u(s, \cdot)$.

We thus need to work with solution spaces for which the hypothesis of this result can be checked. A natural choice is to use a modification of the maximal function $u^{*}$, adapting the one introduced by Kenig-Pipher [27] in the context of elliptic equations:

$$
\tilde{N}(F)(x):=\sup _{\delta>0}\left(f_{\frac{\delta}{2}}^{\delta} f_{B(x, \sqrt{\delta})}|F(t, y)|^{2} \mathrm{~d} y \mathrm{~d} t\right)^{\frac{1}{2}} .
$$

However, note that the space of all measurable functions with $\|\tilde{N}(F)\|_{L^{p}}<\infty$ does not seem to have a trace space at $t=0$, even allowing limits in the weakest possible sense. Hence, finding the initial value relies on the equation as well, using the interior representation above. When $2<p \leq \infty$, we prove well-posedness of global weak solutions of (1.1) in the class $X^{p}=\left\{u \in L_{\text {loc }}^{2}\left(\mathbb{R}_{+}^{n+1}\right) ;\|\tilde{N}(u)\|_{L^{p}}<\infty\right\}$ with arbitrary data in $L^{p}$. In particular, when $p=\infty$, we establish the conservation property

$$
\Gamma(t, 0) 11=11
$$

in $L_{\text {loc }}^{2}\left(\mathbb{R}^{n}\right)$, for all $t>0$. This seems to be new under the sole ellipticity assumption. 
For $p=2$, we also establish, via a different argument, that both $L^{\infty}\left(L^{2}\right)$ and $X^{2}$ are wellposedness classes for $L^{2}$ data. The corresponding solutions agree with the energy solutions. In particular, for any given global weak solution, we have the a priori equivalences

$$
\|u\|_{L^{\infty}\left(L^{2}\right)} \sim\|\nabla u\|_{L^{2}\left(L^{2}\right)} \sim\|\tilde{N}(u)\|_{L^{2} .}
$$

The above results can also be considered for local solutions or for global solutions with growth when $t \rightarrow \infty$. Combining this with the interior representation, we obtain a representation for classes containing global weak solutions having arbitrary growth as $t \rightarrow \infty$ (but still controlled as $|x| \rightarrow \infty$ ). This is quite new as well.

Imposing more properties on the propagators, such as uniform $L^{p}$ boundedness in some range of $p$, allows us to consider the classes $L^{\infty}\left(L^{p}\right)$ as above when $p \neq 2$. This is true for small perturbations of autonomous equations (coefficients independent of $t$ ) or when the coefficients are of bounded variation in time. This is far less demanding than the usual Hölder regularity assumption. We expect that this will give substantial improvements to maximal regularity results for the associated inhomogeneous non-autonomous problem.

Another consequence is that a pointwise upper Gaussian bound condition on the propagator kernel (as obtained by Aronson for real equations) yields unique determination of weak solutions from their traces at $t=0$ in the classes $L^{\infty}\left(L^{p}\right)$, when $1<p \leq \infty$. Note that this pointwise upper Gaussian bound condition has been characterized in [21] in terms of local $L^{2}-L^{\infty}$ estimates of weak solutions of (1.1) and of the dual backward equation. For $p=1$, we obtain, under this assumption, two criteria to decide whether or not a weak solution in $L^{\infty}\left(L^{1}\right)$ is determined by its trace in $L^{1}$ or in the space of Radon measures. This requires some further regularity on the propagators.

Our work also includes a non-autonomous analog of the Fefferman-Stein equivalence (1.4). Namely we prove that, for all weak solutions of $(1.1)$ of the form $u(t, \cdot)=\Gamma(t, 0) f$ with $f \in L^{2} \cap L^{p}$, we have the a priori comparison

$$
\|\tilde{N}(u)\|_{L^{p}} \sim\|\nabla u\|_{T^{p, 2}}, \quad 1 \leq p<\infty,
$$

In fact, the control of $\|\nabla u\|_{T^{p, 2}}$ by $\|\tilde{N}(u)\|_{L^{p}}$ is valid for any global weak solution and $0<p \leq \infty$ and it is only for the converse that we use the form of the solution.

Finally, we prove Fatou type result on non-tangential almost everywhere convergence at the boundary. To do so, since solutions may not be locally bounded, we replace pointwise values by averages on Whitney regions.

The paper is organised as follows. In Section 2, we recall the definitions of various function spaces and operators used in this article. We also recall results from $[5,10]$ that play a key role here.

In Section 3, we develop a new approach to the $L^{2}$ theory, including well-posedness in the largest possible energy space and, as a consequence, the existence of a contraction operator $L^{2}\left(\mathbb{R}^{n}\right)$, called propagator, that maps the data $h$ to our solution $u$ at time $t$. By restriction, this propagator gives both Lions' energy solution and Aronson's energy solution.

In Section 4, we prove the fundamental a priori estimates for weak solutions (either general weak solutions or energy solutions given by the propagator), including reverse Hölder estimates, and appropriate integrated off-diagonal bounds. The latter are a replacement for the pointwise heat kernel bounds available in the case of real coefficients.

In Section 5, we prove our existence and uniqueness results. This includes the key interior representation result, Theorem 1.2, well-posedness in $L^{\infty}\left(L^{2}\right)$ and in $X^{p}$ for $p>2$, 
and the conservation property. Under an additional assumption on the $L^{p}$ behaviour of the propagators, we prove well posedness in $L^{\infty}\left(L^{p}\right)$ for all $p \in(1, \infty]$.

In Section 6, we show that this additional assumption is satisfied for a range of values of $p$ in two important situations: when $A$ is a small $L^{\infty}$ perturbation of a $t$-independent matrix, and when $A$ is of bounded variation in time. We also show a local result when the dependency with respect to $t$ is continuous.

In Section 7, we complete the picture by showing an $L^{p}$ analogue, for $p \in(1, \infty)$, of the norm estimates available for energy solutions when $p=2$. This is an analogue of FeffermanStein's equivalence of maximal function norms and square function norms in Hardy space theory.

In Section 8, we show non-tangential convergence results to the initial data for our weak solutions.

In Section 9, we focus on $p=1$, assuming that our propagators have pointwise kernel bounds (as in the case of real coefficients). We then get a complete theory for Radon measures as data and solutions in $L^{\infty}\left(L^{1}\right)$, or $L^{1}$ data and solutions in a subspace of $L^{\infty}\left(L^{1}\right)$.

Finally, in Section 10, we mention an easy extension of our results: similar well-posedness results hold for global weak solutions $u$ such that norms (in the corresponding solution space) of $(t, x) \mapsto \mathbb{1}_{(0, T)}(t) u(t, x)$ can grow as $T$ tends to $\infty$. A posteriori, we show that this growth is bounded.

\subsection{Acknowledgments}

This work was supported by the Australian Research Council through Discovery Project DP120103692, and Portal's Future Fellowship FT130100607. A long term visit of Monniaux at the Australian National University played a key role in the completion of the project. This visit was possible thanks to the "Laboratoire International Associé en Analyse et Géométrie" agreement between the French Conseil National de la Recherche Scientifique and the ANU. Portal would also like to thank the Laboratoire de Mathématiques de Besançon, for hosting him as part of its 2014 trimester in "Geometric and Noncommutative Methods in Functional Analysis", during which part of this work was conducted. Auscher and Monniaux were also partially supported by the ANR project "Harmonic analysis at its boundaries" ANR-12BS01-0013.

\section{Preliminaries}

\section{$2.1 \quad$ Function spaces}

\section{Vector valued spaces}

When dealing with function spaces over $(a, b) \times \mathbb{R}^{n}$, we write $L^{p}(X)$ for the Bochner space of $X\left(\mathbb{R}^{n}\right)$ valued $L^{p}$ functions $L^{p}\left(a, b ; X\left(\mathbb{R}^{n}\right)\right)$ or $L^{p}\left(a, b ; X\left(\mathbb{R}^{n} ; \mathbb{C}^{n}\right)\right.$ ) (as long as no confusion can occur).

We denote by $\mathscr{D}$ the space $\mathscr{C}_{c}^{\infty}\left((0, \infty) \times \mathbb{R}^{n}\right)$ and by $\mathscr{D}^{\prime}$ the space of distributions on $(0, \infty) \times \mathbb{R}^{n}$. We denote by $\mathscr{C}_{0}\left(L^{p}\right)$ the space of $L^{p}\left(\mathbb{R}^{n}\right)$-valued continuous functions on $[0, \infty)$ that tend to 0 at infinity. 


\section{The homogeneous Sobolev spaces $\dot{H}^{1}\left(\mathbb{R}^{n}\right)$}

There are many ways to define the homogeneous space $\dot{H}^{1}\left(\mathbb{R}^{n}\right)$. We depart a little bit from tradition of having this space as a space of distributions modulo constants, as this simplifies its use in (1.1).

We denote by $H^{s}\left(\mathbb{R}^{n}\right)$ the standard inhomogeneous Sobolev space for $s \in \mathbb{R}$, and we equip $L^{2}\left(\mathbb{R}^{n} ; \mathbb{C}^{k}\right)$ with the standard complex inner product, which we denote by $\langle\cdot, \cdot\rangle$ or $L^{2}\langle\cdot, \cdot\rangle_{L^{2}}$.

We set $\dot{H}^{1}\left(\mathbb{R}^{n}\right)=\left\{u \in \mathscr{D}^{\prime}\left(\mathbb{R}^{n}\right) ; \nabla u \in L^{2}\left(\mathbb{R}^{n} ; \mathbb{C}^{n}\right)\right\}$, and equip this space with the seminorm $u \mapsto\|\nabla u\|_{L^{2}}$. With this definition, the following properties hold:

1. $H^{1}\left(\mathbb{R}^{n}\right) \subset \dot{H}^{1}\left(\mathbb{R}^{n}\right) \subset L_{\text {loc }}^{2}\left(\mathbb{R}^{n}\right)$ (set inclusions).

2. $\mathscr{D}\left(\mathbb{R}^{n}\right)$ is dense in $\dot{H}^{1}\left(\mathbb{R}^{n}\right)$ : for all $u \in \dot{H}^{1}\left(\mathbb{R}^{n}\right)$ there exists a sequence $\left(\phi_{j}\right)_{j \in \mathbb{N}}$ of functions in $\mathscr{D}\left(\mathbb{R}^{n}\right)$ such that $\left\|\nabla \phi_{j}-\nabla u\right\|_{2} \underset{j \rightarrow \infty}{\longrightarrow} 0$.

3. $\dot{H}^{1}\left(\mathbb{R}^{n}\right) / \mathbb{C}$ is a Banach space equipped with its quotient norm.

4. $\dot{H}^{1}\left(\mathbb{R}^{n}\right) \subset \mathscr{S}^{\prime}\left(\mathbb{R}^{n}\right)$ (set inclusion).

5. The dual of $\dot{H}^{1}\left(\mathbb{R}^{n}\right)$ can be identified with the dual of $\dot{H}^{1}\left(\mathbb{R}^{n}\right) / \mathbb{C}$, and with $\dot{H}^{-1}\left(\mathbb{R}^{n}\right)=$ $\left\{\operatorname{div} g ; g \in L^{2}\left(\mathbb{R}^{n} ; \mathbb{C}^{n}\right)\right\}$ equipped with the norm $f \mapsto\|f\|_{\dot{H}^{-1}}=\inf \left\{\|g\|_{L^{2}} ; f=\right.$ $\operatorname{div} g$ \}. Moreover, for all $u \in \dot{H}^{1}\left(\mathbb{R}^{n}\right)$, all $g \in L^{2}\left(\mathbb{R}^{n}, \mathbb{C}^{n}\right)$, and $f=\operatorname{div} g$, we have that

$$
\dot{H}^{-1}\langle f, u\rangle_{\dot{H}^{1}}=-L_{L^{2}}\langle g, \nabla u\rangle_{L^{2}}={ }_{\dot{H}^{-1}}\langle f,[u]\rangle_{\dot{H}^{1} / \mathbb{C}} .
$$

In particular, $\dot{H}^{-1}\left(\mathbb{R}^{n}\right) \subset H^{-1}\left(\mathbb{R}^{n}\right) \subset \mathscr{S}^{\prime}\left(\mathbb{R}^{n}\right)$ (embeddings), and, if $u \in \dot{H}^{1}\left(\mathbb{R}^{n}\right) \cap$ $L^{2}\left(\mathbb{R}^{n}\right)=H^{1}\left(\mathbb{R}^{n}\right)$ and $f \in \dot{H}^{-1}\left(\mathbb{R}^{n}\right) \cap L^{2}\left(\mathbb{R}^{n}\right)$ then

$$
\dot{H}^{-1}\langle f, u\rangle_{\dot{H}^{1}}=\int_{\mathbb{R}^{n}} f(x) \overline{u(x)} \mathrm{d} x={ }_{L^{2}}\langle f, u\rangle_{L^{2}} .
$$

These properties are well known. We shall often write $\dot{H}^{1}\langle u, f\rangle_{\dot{H}^{-1}}$ to mean $\dot{H}^{-1} \overline{\langle f, u\rangle}_{\dot{H}^{1}}$. Having this in hand, we have that, for $A$ satisfying (1.2) and almost every $t>0$,

$$
L(t)=-\operatorname{div} A(t, \cdot) \nabla
$$

defines a bounded operator from $\dot{H}^{1}\left(\mathbb{R}^{n}\right)$ to $\dot{H}^{-1}\left(\mathbb{R}^{n}\right)$, which is onto and has $\mathbb{C}$ as its null space (if one uses $\dot{H}^{1}\left(\mathbb{R}^{n}\right) / \mathbb{C}$, we thus have an isomorphism). More precisely, for all $u, v \in \dot{H}^{1}\left(\mathbb{R}^{n}\right), \dot{H}^{1}\left\langle L(t) u, v{\dot{H^{-1}}}={ }_{L^{2}}\langle A(t, .) \nabla u, \nabla v\rangle_{L^{2}}\right.$, and

$$
\lambda\|u\|_{\dot{H}^{1}} \leq\|L(t) u\|_{\dot{H}^{-1}} \leq \Lambda\|u\|_{\dot{H}^{1}} .
$$

Now assume that $A$ is constant in $t$, and set $L=-\operatorname{div} A \nabla$ and $D(L)=\left\{u \in H^{1} ; L u \in L^{2}\right\}$. Then $L$ is the maximal accretive operator on $L^{2}\left(\mathbb{R}^{n}\right)$ associated with the form $(u, v) \mapsto$ $L^{2}\langle A \nabla u, \nabla v\rangle_{L^{2}}$ on $H^{1}\left(\mathbb{R}^{n}\right)$. In particular, it is sectorial and $-L$ generates an analytic semigroup of contractions $\left(e^{-t L}\right)_{t>0}$. Also, the solution of Kato's square root conjecture in [8] implies that

$$
\sup _{t>0}\left\|\nabla e^{-t L} u\right\|_{L^{2}} \lesssim \sup _{t>0}\left\|L^{\frac{1}{2}} e^{-t L} u\right\|_{L^{2}} \lesssim\left\|L^{\frac{1}{2}} u\right\|_{L^{2}} \lesssim\|\nabla u\|_{L^{2}} \quad \forall u \in H^{1}\left(\mathbb{R}^{n}\right) .
$$


Therefore, as $e^{-t L} 11=11$ in $L_{\text {loc }}^{2}$ (see $[5, \S 2.5]$ ), we have that $\left\{e^{-t L} ; t>0\right\}$ extends to a uniformly bounded family of bounded operators on $\dot{H}^{1}\left(\mathbb{R}^{n}\right)$. Finally, we use the space $L^{2}\left(a, b ; \dot{H}^{1}\left(\mathbb{R}^{n}\right)\right)$ for $-\infty \leq a<b \leq+\infty$, endowed with the seminorm $u \mapsto\left(\int_{a}^{b}\|\nabla u(t, .)\|_{L^{2}}^{2} \mathrm{~d} t\right)^{\frac{1}{2}}$. It follows from the above discussion that $\mathscr{C}_{c}^{\infty}\left((a, b) \times \mathbb{R}^{n}\right)$ is dense in $L^{2}\left(a, b ; \dot{H}^{1}\left(\mathbb{R}^{n}\right)\right)$, that $L^{2}\left(a, b ; \dot{H}^{1}\left(\mathbb{R}^{n}\right)\right) \subset L^{2}\left(a, b ; L_{\text {loc }}^{2}\left(\mathbb{R}^{n}\right) \cap \mathscr{S}^{\prime}\left(\mathbb{R}^{n}\right)\right)$, and that its dual can be identified with $L^{2}\left(a, b ; \dot{H}^{-1}\left(\mathbb{R}^{n}\right)\right)$ through the pairing

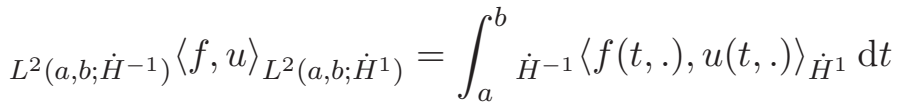

$$
\begin{aligned}
& =-\int_{a}^{b} L^{2}\langle\psi(t, .), \nabla u(t, .)\rangle_{L^{2}} \mathrm{~d} t,
\end{aligned}
$$

for any $\psi \in L^{2}\left(a, b ; L^{2}\left(\mathbb{R}^{n}\right)\right)$ such that $f=\operatorname{div} \psi$, and $u \in L^{2}\left(a, b ; \dot{H}^{1}\left(\mathbb{R}^{n}\right)\right)$.

\section{Homogeneous Lions spaces $\dot{W}(0, \infty)$}

We define the following spaces that are variants of the solution spaces used by Lions in [30, spaces $\mathscr{A}(\Omega)$ and $\mathscr{B}(\Omega)$ p. 147] (see also [16, Chap. XVIII]).

$$
\begin{aligned}
& \dot{W}(0, \infty):=\left\{u \in \mathscr{D}^{\prime} ; u \in L^{2}\left(\dot{H}^{1}\right) \text { and } \partial_{t} u \in L^{2}\left(\dot{H}^{-1}\right)\right\} \\
& \text { and } \\
& W(0, \infty):=\dot{W}(0, \infty) \cap \mathscr{C}_{0}\left(L^{2}\right),
\end{aligned}
$$

and the corresponding spaces on a time interval $(a, b), 0 \leq a<b<\infty$

$$
\dot{W}(a, b):=\left\{u \in\left(\mathscr{C}_{c}^{\infty}\left((a, b) \times \mathbb{R}^{n}\right)\right)^{\prime} ; u \in L^{2}\left(a, b ; \dot{H}^{1}\right) \text { and } \partial_{t} u \in L^{2}\left(a, b ; \dot{H}^{-1}\right)\right\},
$$

and $W(a, b)=\dot{W}(a, b) \cap \mathscr{C}\left([a, b] ; L^{2}\right)$. An important result of Lions [30, Proposition 3.1] states that inhomogeneous versions of these spaces (replacing $\dot{H}^{1}$ and $\dot{H}^{-1}$ by $H^{1}$ and $H^{-1}$ ) embed into $\mathscr{C}\left([a, b] ; L^{2}\right)$, (see also [16, Chap. XVIII]), that is, into $W(a, b)$. With quite a different proof, we prove, in Section 3.1, a version of this result for $\dot{W}(0, \infty)$.

\section{Tent spaces $T^{p, 2}$}

The tent spaces introduced by Coifman, Meyer, and Stein in [15] play a key role in our work. For $p \in(0, \infty]$, the (parabolic) tent space $T^{p, 2}$ is the set of measurable functions $u$ on $\mathbb{R}_{+}^{n+1}$ such that

$$
\begin{aligned}
& x \mapsto\left(\int_{0}^{\infty} f_{B(x, \sqrt{t})}|u(t, y)|^{2} \mathrm{~d} y \mathrm{~d} t\right)^{\frac{1}{2}} \in L^{p}\left(\mathbb{R}^{n}\right), \text { if } p<\infty, \\
& x \mapsto \sup _{B \ni x}\left(\int_{0}^{r_{B}^{2}} f_{B}|u(t, y)|^{2} \mathrm{~d} y \mathrm{~d} t\right)^{\frac{1}{2}} \in L^{\infty}\left(\mathbb{R}^{n}\right), \text { if } p=\infty,
\end{aligned}
$$

where we denote by $r_{B}$ the radius of a ball $B$. Note that $T^{p, 2}$ is contained in $L_{\text {loc }}^{2}\left(\mathbb{R}_{+}^{n+1}\right)$. As shown in [15], these spaces are Banach spaces when $1 \leq p \leq \infty$, reflexive when $p \in$ $(1, \infty)$, and the dual of $T^{p, 2}$ is $T^{p^{\prime}, 2}$ for the duality given by $\int_{\mathbb{R}_{+}^{n+1}} f(t, y) g(t, y) \mathrm{d} y \mathrm{~d} t$. Their importance for us has two origins. One is elliptic boundary value problems including the Laplace equation, where tent spaces, along with closely related objects such as Hardy spaces 
and Carleson measures, are already used extensively. Since we consider equation (1.1) weakly in space and time, it is natural to use such norms rather than the $L^{\infty}\left(L^{p}\right)$ norms which would correspond to treating (1.1) as an (non-autonomous) evolution equation in $L^{p}$. The other reason why tent spaces are so important in our work comes from the recent extension of Calderón-Zygmund theory to rough settings, i.e. the application of CalderónZygmund ideas to operators such as $e^{-t L}$ with $L=-\operatorname{div} A \nabla, A \in L^{\infty}\left(\mathbb{R}^{n} ; \mathscr{M}_{n}(\mathbb{C})\right)$ satifying (1.2), that do not, in general, have Calderón-Zygmund kernels (see [5] and the references therein). In such a setting, integral operators such as

$$
f \mapsto\left[(t, x) \mapsto \int_{0}^{t} \nabla e^{-(t-s) L} \operatorname{div} f(s, \cdot)(x) \mathrm{d} s\right]
$$

are often unbounded on Bochner spaces $L^{p}\left(L^{q}\right)$ but bounded on $T^{p, 2}$. This is the subject of our paper [10]. The results we use here are recalled in Section 2.2. Keeping in mind that $T^{2,2}=L^{2}\left(L^{2}\right)$, we then use the condition $\nabla u \in T^{p, 2}$ (here, we mean that each component of $\nabla u$ is in $T^{p, 2}$; in general, we shall not distinguish the notation as this will be clear from the context) as a replacement for the condition $\nabla u \in L^{2}\left(L^{2}\right)$ to attack $L^{p}$ theory. For uniqueness, however, maximal function estimates on solutions are more suitable than square function estimates.

\section{Kenig-Pipher modified $T^{p, \infty}$ space $X^{p}$}

Coifman-Meyer-Stein's tent space theory also includes maximal function estimates via the tent spaces $T^{p, \infty}$ defined as spaces of continuous functions on $(0, \infty) \times \mathbb{R}^{n}$ with $u^{*} \in L^{p}$ and with non-tangential limit, where $u^{*}$ is the non-tangential maximal function defined by

$$
u^{*}: x \mapsto \sup _{\substack{(t, y) \in(0, \infty) \times \mathbb{R}^{n} \\|x-y|<\sqrt{t}}}|u(t, y)| .
$$

This maximal function, however, is not appropriate for us because of the lack of pointwise bounds on our solutions. We thus use a modified version of the non-tangential maximal function, introduced by Kenig and Pipher for elliptic equations in [27], and used extensively in [7] (see also [22, 11] and further development in the theory of Hardy spaces associated with operators without Gaussian bounds).

Definition 2.1. For $F \in L_{\text {loc }}^{2}\left(\mathbb{R}_{+}^{n+1}\right)$, we define the following maximal function $\tilde{N}(F)$ by

$$
\tilde{N}(F)(x):=\sup _{\delta>0}\left(f_{\frac{\delta}{2}}^{\delta} f_{B(x, \sqrt{\delta})}|F(t, y)|^{2} \mathrm{~d} y \mathrm{~d} t\right)^{\frac{1}{2}}, \quad \forall x \in \mathbb{R}^{n} .
$$

The corresponding modification of $T^{p, \infty}$ is defined as follows.

Definition 2.2. Let $0<p \leq \infty$. The space $X^{p}$ is the subspace of functions $F \in L_{\text {loc }}^{2}\left(\mathbb{R}_{+}^{n+1}\right)$ such that

$$
\|F\|_{X^{p}}:=\|\tilde{N}(F)\|_{p}<\infty .
$$

This space has been defined in [27]. For $1 \leq p \leq \infty$, it is a Banach space. Duality and interpolation is studied in [24, 23].

Note that, given a parameter $\beta>1$, the maximal function $\tilde{N}(F)$ in the definitions above can be replaced by

$$
\mathcal{N}_{\beta}(F)(x)=\sup _{\delta>0}\left(f_{\delta^{2}}^{\beta^{2} \delta^{2}} f_{B(x, \beta \delta)}|F(t, y)|^{2} \mathrm{~d} y \mathrm{~d} t\right)^{\frac{1}{2}}, \quad \forall x \in \mathbb{R}^{n},
$$


since a simple covering argument yields $\left\|\mathcal{N}_{\beta}(F)\right\|_{p} \sim\|F\|_{X^{p}}$.

A difficulty with this norm compared to the one with $u^{*}$ is the lack of stability by translation: one can check that if $\tau_{s} F(t, x)=F(t+s, x)$, then there is neither pointwise nor $L^{p}$ control of $\tilde{N}\left(F_{s}\right)$ by $\tilde{N}(F)$ for any $p$. The same difficulty appears with the tent spaces $T^{p, 2}$ above except when $p=2$.

\section{Slice spaces $E_{\delta}^{p}$}

While integral operators such as

$$
f \mapsto\left[(t, x) \mapsto \int_{0}^{t} \nabla e^{-(t-s) L} \operatorname{div} f(s, \cdot)(x) \mathrm{d} s\right]
$$

act on $T^{p, 2}$ (see Section 2.2), their (operator-valued) kernels $\nabla e^{-t L} \operatorname{div}$ (for a fixed $t>0$ ) do not act, in general, on $L^{p}\left(\mathbb{R}^{n}\right)$. Appropriate substitutes for $L^{p}\left(\mathbb{R}^{n}\right)$ are the following spaces.

Definition 2.3. Let $p \in[1, \infty]$ and $\delta>0$. The (parabolic) slice space $E_{\delta}^{p}$ is the subspace of functions $g \in L_{\text {loc }}^{2}\left(\mathbb{R}^{n}\right)$ such that

$$
\|g\|_{E_{\delta}^{p}}:=\left(\int_{\mathbb{R}^{n}}\left(f_{B(x, \sqrt{\delta})}|g(y)|^{2} \mathrm{~d} y\right)^{\frac{p}{2}} \mathrm{~d} x\right)^{\frac{1}{p}}<\infty .
$$

This space can also be seen as one of Wiener amalgam spaces, which have been studied for a long time. However, $[12, \S 3]$ points out that these spaces are retracts of tent spaces, and thus inherit many of their key properties: $\left(E_{\delta}^{p}\right)^{*}=E_{\delta}^{p^{\prime}}$ with $\|\ell\|_{\left(E_{\delta}^{p}\right)^{*}} \sim\|\ell\|_{E_{\delta}^{p^{\prime}}}$, with implicit constants uniform in $\delta>0$, for all $p \in[1, \infty)$ under the duality pairing $\int_{\mathbb{R}^{n}} f(x) \overline{g(x)} \mathrm{d} x$. In particular, slice spaces are reflexive Banach spaces when $p \in(1, \infty)$. The following result is [12, Lemma 3.5] and compares the norms in $E_{\delta}^{p}$ and in $E_{\delta^{\prime}}^{p}$ for $\delta^{\prime} \neq \delta$.

Lemma 2.4. Let $p \in[1, \infty]$ and $\delta, \delta^{\prime}>0$. For all $f \in E_{\delta^{\prime}}^{p}$, one has $f \in E_{\delta}^{p}$ and

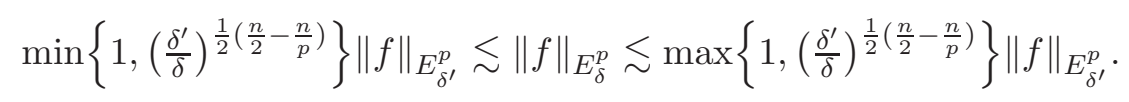

\subsection{Maximal regularity operators}

Given $A \in L^{\infty}\left(\mathbb{R}^{n} ; \mathscr{M}_{n}(\mathbb{C})\right)$ satisfying (1.2), recall that $L=-\operatorname{div} A \nabla$ denotes the maximal accretive operator with domain $D(L)=\left\{u \in H^{1}\left(\mathbb{R}^{n}\right) ; A \nabla u \in D(\right.$ div $\left.)\right\}$. Recall also that $\langle L u, v\rangle=\langle A \nabla u, \nabla v\rangle$ for all $u \in D(L)$ and $v \in H^{1}\left(\mathbb{R}^{n}\right)$. See [5, 34], for more background on the operator theory of divergence form elliptic operators.

We consider the associated maximal regularity operator $\mathcal{M}_{L}$ initially defined as a bounded operator from $L^{1}(D(L))$ to $L_{\mathrm{loc}}^{\infty}\left(L^{2}\right)$ by

$$
\mathcal{M}_{L} f(t, x)=\int_{0}^{t} L e^{-(t-s) L} f(s, \cdot)(x) \mathrm{d} s
$$

for almost every $(t, x) \in(0, \infty) \times \mathbb{R}^{n}$ and all $f \in L^{1}(D(L))$. A classical result by De Simon [17] states that $\mathcal{M}_{L}$ extends to a bounded operator on $L^{2}\left(L^{2}\right)$.

De Simon's result can be extended in several directions, including $L^{p}\left(L^{p}\right)$ boundedness, $L^{p}\left(\mathbb{R}^{n} ; L^{2}(0, \infty)\right)$ boundedness, and $T^{p, 2}$ boundedness. 
The $L^{p}\left(L^{p}\right)$ extension is the most well-known. Lutz Weis proved in [37] that the maximal regularity operator $\mathcal{M}_{L}$ belongs to $\mathscr{L}\left(L^{p}\left(L^{p}\right)\right)$ if and only if $\left(e^{-t L}\right)_{t \geq 0}$ is $R$-analytic in $L^{p}\left(\mathbb{R}^{n}\right)$. This holds in a range $\left(p_{-}(L), p_{+}(L)\right)$ around 2 as shown in [5, Theorem 5.1] (combined with [26, Theorem 5.3]). Note that, for $p$ outside of $\left[p_{-}(L), p_{+}(L)\right],-L$ does not generate a $\mathscr{C}_{0}$-semigroup on $L^{p}$.

The $L^{p}\left(\mathbb{R}^{n} ; L^{2}(0, \infty)\right)$ extension has recently been considered in [36]. Again $\mathcal{M}_{L} \in$ $\mathscr{L}\left(L^{p}\left(\mathbb{R}^{n} ; L^{2}(0, \infty)\right)\right)$ when $p \in\left(p_{-}(L), p_{+}(L)\right)$ by a combination of [5, Theorem 5.1] and [36, Theorem 3.3].

The $T^{p, 2}$ extension is the subject of our work [10]. In [10, Proposition 1.6], we prove that $\mathcal{M}_{L} \in \mathscr{L}\left(T^{p, 2}\right)$ for a range of values of $p$ that can be strictly larger than $\left(p_{-}(L), p_{+}(L)\right)$ (recall that, for all $\varepsilon>0$, there exists $-L$ that does not generate a semigroup on $L^{p}\left(\mathbb{R}^{n}\right)$ for $\left.p<\frac{2 n}{n+2}-\varepsilon\right)$.

In this paper, however, we need to use a variant $\tilde{\mathcal{M}}_{L}$ of $\mathcal{M}_{L}$ for which the $T^{p, 2}$ boundedness has still a large range of exponents while the $L^{p}\left(L^{p}\right)$ theory would hold on an even smaller range than for $\mathcal{M}_{L}$.

Proposition 2.5. The integral

$$
\tilde{\mathcal{M}}_{L} f(t, \cdot)=\int_{0}^{t} \nabla e^{-(t-s) L} \operatorname{div} f(s, \cdot) \mathrm{d} s
$$

defines a bounded operator from $L^{1}\left(H^{2}\right)$, where $H^{2}=H^{2}\left(\mathbb{R}^{n} ; \mathbb{C}^{n}\right)$ ), to $L_{\text {loc }}^{\infty}\left(L^{2}\right)$. This operator extends to a bounded operator on $L^{2}\left(L^{2}\right)$.

Proof. To see that $\tilde{\mathcal{M}}_{L}$ is well defined, remark that, for all $\tau>0$ and all $g \in H^{2}$,

$$
\left\|\nabla e^{-\tau L} \operatorname{div} g\right\|_{L^{2}} \lesssim\|\nabla \operatorname{div} g\|_{L^{2}} \lesssim\|g\|_{H^{2}} .
$$

Next, we turn to the extension. Remark that for such $g, h=L^{-\frac{1}{2}} \operatorname{div} g \in D(L)$. First, $g \in L^{2}\left(\mathbb{R}^{n} ; \mathbb{C}^{n}\right)$ and by the solution of the Kato square root problem [8], $h \in L^{2}\left(\mathbb{R}^{n}\right)$. Secondly, $L h=L^{\frac{1}{2}} \operatorname{div} g \in L^{2}\left(\mathbb{R}^{n}\right)$ as $\operatorname{div} g \in H^{1}=D\left(L^{\frac{1}{2}}\right)$ by [8]. Using $L^{2}$ boundedness of $\nabla L^{-\frac{1}{2}},[8]$, we have the equality in $L^{2}$

$$
\nabla e^{-\tau L} \operatorname{div} g=\nabla L^{-\frac{1}{2}} L e^{-\tau L} L^{-\frac{1}{2}} \operatorname{div} g
$$

for all such $g$ and all $\tau>0$. It follows

$$
\tilde{\mathcal{M}}_{L} f=\nabla L^{-\frac{1}{2}} \mathcal{M}_{L} L^{-\frac{1}{2}} \operatorname{div} f
$$

for all $f \in L^{1}\left(H^{2}\right)$ and that $\tilde{\mathcal{M}}_{L}$ extends by density to a bounded operator on $L^{2}\left(L^{2}\right)$.

The adjoint $\tilde{\mathcal{M}}_{L}^{*} \in \mathscr{L}\left(L^{2}\left(L^{2}\right)\right)$ is given as follows.

Lemma 2.6. For all $f, g \in \mathscr{D}$,

$$
\int_{\mathbb{R}}\left\langle\tilde{\mathcal{M}}_{L} f(t, \cdot), g(t, \cdot)\right\rangle \mathrm{d} t=\int_{\mathbb{R}}\left\langle f(t, \cdot), \int_{0}^{\infty} \nabla\left(e^{-s L}\right)^{*} \operatorname{div} g(t+s, \cdot) \mathrm{d} s\right\rangle \mathrm{d} t .
$$

Proof. Let $f, g \in \mathscr{D}$. We have that

$$
\begin{aligned}
\int_{\mathbb{R}}\left\langle\tilde{\mathcal{M}}_{L} f(t, \cdot), g(t, \cdot)\right\rangle \mathrm{d} t & =\int_{\mathbb{R}} \int_{\mathbb{R}} \mathbb{1}_{(0, \infty)}(t-s)\left\langle\nabla e^{-(t-s) L} \operatorname{div} f(s, \cdot), g(t, \cdot)\right\rangle \mathrm{d} s \mathrm{~d} t \\
& =\int_{\mathbb{R}} \int_{\mathbb{R}} \mathbb{1}_{(0, \infty)}(\sigma)\left\langle f(s, \cdot), \nabla\left(e^{-\sigma L}\right)^{*} \operatorname{div} g(\sigma+s, \cdot)\right\rangle \mathrm{d} \sigma \mathrm{d} s
\end{aligned}
$$

where we have made the change of variables $s=s$ and $\sigma=t-s$ on $\mathbb{R} \times \mathbb{R}$. 
Remark 2.7. The operator, initially defined for $g \in \mathscr{D}$ by

$$
\tilde{\mathcal{M}}_{L}^{*} g(s, x)=\int_{0}^{\infty} \nabla\left(e^{-\sigma L}\right)^{*} \operatorname{div} g(\sigma+s, \cdot)(x) \mathrm{d} \sigma, \quad(s, x) \in(0, \infty) \times \mathbb{R}^{n}
$$

thus extends to a bounded linear operator on $L^{2}\left(L^{2}\right)$.

Proposition 2.8. Let $q \in[1,2)$ be such that $\sup _{t>0}\left\|\sqrt{t} \nabla e^{-t L^{*}}\right\|_{\mathscr{L}\left(L^{s}\right)}<\infty$ for all $s \in$ $\left[2, q^{\prime}\right)$. Then $\tilde{\mathcal{M}}_{L}$ extends to a bounded operator on $T^{p, 2}$ for all $p \in\left(p_{c}, \infty\right]$ where $p_{c}=$ $\max \left\{\frac{n q}{n+q}, \frac{2 n}{n+q^{\prime}}\right\}$.

Proof. We first recall, from [5, Section 3.4], that there exist an exponent $q$ as above (denoted by $q_{+}\left(L^{*}\right)^{\prime}$ in [5]), and another one $p_{-}(L) \geq 1$ with $p_{-}(L) \leq \max \left\{1, \frac{n q}{n+q}\right\}$ such that

$$
\sup _{t \geq 0}\left\|e^{-t L}\right\|_{\mathscr{L}\left(L^{r}\right)}<\infty, \quad \forall r \in\left(p_{-}(L), 2\right] .
$$

To prove the result for $p \leq 2$, we apply [10, Theorem 3.1] with $m=2, \beta=0$. To do so, we only have to show that

$$
\sup _{t>0}\left\|t^{1+\frac{n}{2}\left(\frac{1}{\tilde{q}}-\frac{1}{2}\right)} \nabla e^{-t L} \operatorname{div}\right\|_{\mathscr{L}\left(L^{\tilde{q}}, L^{2}\right)}<\infty
$$

for all $\tilde{q} \in(q, 2]$ and compute the exponents. Indeed, this estimate and $L^{2}-L^{2}$ off diagonal estimates imply the $L^{r}-L^{2}$ decay with $r \in(\tilde{q}, 2)$. See for example [5, Proposition 3.2]. Write

$$
t^{1+\frac{n}{2}\left(\frac{1}{\tilde{q}}-\frac{1}{2}\right)} \nabla e^{-t L} \operatorname{div}=A_{t} B_{t} C_{t}
$$

with $A_{t}=t^{\frac{1}{2}} \nabla e^{-\frac{t}{3} L}, B_{t}=t^{\frac{n}{2}\left(\frac{1}{\tilde{q}}-\frac{1}{2}\right)} e^{-\frac{t}{3} L}$ and $C_{t}=t^{\frac{1}{2}} e^{-\frac{t}{3} L}$ div. Observe that $C_{t}$ is uniformly bounded on $L^{\tilde{q}}$ using $\tilde{q}>q$ and duality. Next, $B_{t}$ is uniformly bounded from $L^{\tilde{q}}$ to $L^{2}$ by [5, Proposition 3.9] and (2.3). Finally $A_{t}$ is uniformly bounded on $L^{2}$. For $p \geq 2$, we apply [10, Proposition 4.2] with $m=2, \beta=0$ and $q=2$.

Remark 2.9. If we were to use maximal regularity results in $L^{p}\left(L^{p}\right)$ or $L^{p}\left(\mathbb{R}^{n} ; L^{2}(0, \infty)\right)$ as in [36] instead of this result, we would need the family $\left\{\nabla e^{-t L}\right.$ div ; $\left.t>0\right\}$ to be $R$-bounded on $L^{p}\left(\mathbb{R}^{n}\right)$. As shown in [5], this is false for $p<q$, and $q$ can be arbitrarily close to 2 . In the above proposition, however, we allow, at least, $p \in\left[\frac{2 n}{n+2}, \infty\right]$ (see $\left.[5,9]\right)$.

Remark 2.10. If $q^{\prime}>n$, then $p_{c}=\frac{n q}{n+q}<1$. When $q^{\prime} \leq n, p_{c}=\frac{2 n}{n+q^{\prime}}$. Actually, we have learned from Yi Huang (personal communication) that in this case, the exponent $p_{c}$ can be taken to be the smaller value $\frac{n q}{n+q}$, using an improved version of [10, Theorem 3.1]. This value is in agreement with the number $p_{-}(L)$ above.

Remark 2.11. We remark that given the ellipticity constants $\lambda$, $\Lambda$, there is $\varepsilon(\lambda, \Lambda) \in(0, \infty]$ such that $q_{+}\left(L^{*}\right) \geq 2+\varepsilon(\lambda, \Lambda)$ whenever $A$ satisfies (1.2). This implies that $p_{-}(L) \leq$ $\max \left\{1, \frac{2 n}{n+2}-\varepsilon^{\prime}(\lambda, \Lambda, n)\right\}$ for such $L$. See again [5, Section 3.4].

We also consider the integral operator $\mathcal{R}_{L}$ initially defined as a bounded operator from $L^{1}\left(H^{1}\right)$, with $H^{1}=H^{1}\left(\mathbb{R}^{n} ; \mathbb{C}^{n}\right)$, to $L_{\mathrm{loc}}^{\infty}\left(L^{2}\right)$ by

$$
\mathcal{R}_{L} f(t, x)=\int_{0}^{t} e^{-(t-s) L} \operatorname{div} f(s, \cdot)(x) \mathrm{d} s .
$$

Note that $\mathscr{C}_{c}\left(\mathbb{R}_{+}^{n+1} ; \mathbb{C}^{n}\right)$, the space of compactly supported continuous functions on $\mathbb{R}_{+}^{n+1}$ into $\mathbb{C}^{n}$ is contained in $L^{1}\left(H^{1}\right)$ and is dense in $T^{p, 2}$ (of $\mathbb{C}^{n}$-valued functions) for all $p \in$ $(0, \infty)$. 
Proposition 2.12. Let $p \in(0, \infty]$. The operator $\mathcal{R}_{L}$ extends to a bounded operator from $T^{p, 2}$ to $X^{p}$.

In the proof below, and throughout the paper, we use dyadic annuli defined as follows. For $x \in \mathbb{R}^{n}, r>0$, set $S_{1}(x, r)=B(x, 2 r)$, and $S_{j}(x, r)=B\left(x, 2^{j+1} r\right) \backslash B\left(x, 2^{j} r\right)$ for $j \geq 2$.

Proof. Let $f \in \mathscr{C}_{c}\left(\mathbb{R}_{+}^{n+1} ; \mathbb{C}^{n}\right)$. We have that, for almost every $(t, x) \in \mathbb{R}_{+}^{n+1}$,

$$
\mathcal{R}_{L} f(t, x)=\sum_{k=0}^{\infty} e^{-\left(1-2^{-k}\right) t L} K_{L} f\left(2^{-k} t, x\right)
$$

where $K_{L} f(t, x)=\int_{\frac{t}{2}}^{t} e^{-(t-s) L} \operatorname{div} f(s, \cdot)(x) \mathrm{d} s$. Fix $x \in \mathbb{R}^{n}$ and $k \in \mathbb{N} \backslash\{0\}$. Since $\left\{e^{-t L} ; t \geq 0\right\}$ satisfies Gaffney-Davies estimates (see $[5, \S 2.3]$ ), we have that for any $\delta>0$,

$$
\begin{aligned}
\left(f_{\frac{\delta}{2}}^{\delta}\right. & \left.f_{B(x, \sqrt{\delta})}\left|e^{-\left(1-2^{-k}\right) t L} K_{L} f\left(2^{-k} t, y\right)\right|^{2} \mathrm{~d} y \mathrm{~d} t\right)^{\frac{1}{2}} \\
& \leq \sum_{j=1}^{\infty}\left(f_{\frac{\delta}{2}}^{\delta} f_{B(x, \sqrt{\delta})}\left|e^{-\left(1-2^{-k}\right) t L}\left(1_{S_{j}(x, \sqrt{\delta})} K_{L} f\left(2^{-k} t, \cdot\right)\right)(y)\right|^{2} \mathrm{~d} y \mathrm{~d} t\right)^{\frac{1}{2}} \\
& \lesssim \sum_{j=1}^{\infty} 2^{j \frac{n}{2}} e^{-c 4^{j}}\left(f_{\frac{\delta}{2}}^{\delta} f_{B\left(x, 2^{j+1} \sqrt{\delta}\right)}\left|K_{L} f\left(2^{-k} t, y\right)\right|^{2} \mathrm{~d} y \mathrm{~d} t\right)^{\frac{1}{2}} \\
& \leq \sum_{j=1}^{\infty} 2^{j \frac{n}{2}} e^{-c 4^{j}} \sup _{\delta^{\prime}>0}\left(f_{\frac{2^{k} \delta^{\prime}}{2}}^{2^{k} \delta^{\prime}} f_{B\left(x, 2^{\left.j+1+\frac{k}{2} \sqrt{\delta^{\prime}}\right)}\right.}\left|K_{L} f\left(2^{-k} t, y\right)\right|^{2} \mathrm{~d} y \mathrm{~d} t\right)^{\frac{1}{2}} \\
& =\sum_{j=1}^{\infty} 2^{j \frac{n}{2}} e^{-c 4^{j}} \sup _{\delta^{\prime}>0}\left(f_{\frac{\delta^{\prime}}{2}}^{\delta^{\prime}} f_{B\left(x, 2^{\left.j+1+\frac{k}{2} \sqrt{\delta^{\prime}}\right)}\right.}\left|K_{L} f(t, y)\right|^{2} \mathrm{~d} y \mathrm{~d} t\right)^{\frac{1}{2}} .
\end{aligned}
$$

Note that this estimate also holds for $k=0$. Now with $\delta^{\prime}>0$, and $j \geq 1$, we have that

$$
\begin{aligned}
& \left(f_{\frac{\delta^{\prime}}{2}}^{\delta^{\prime}} f_{B\left(x, 2^{\left.j+1+\frac{k}{2} \sqrt{\delta^{\prime}}\right)}\right.}\left|K_{L} f(t, y)\right|^{2} \mathrm{~d} y \mathrm{~d} t\right)^{\frac{1}{2}} \\
& \quad \leq \sum_{\ell=1}^{\infty}\left(f_{\frac{\delta^{\prime}}{2}}^{\delta^{\prime}} f_{B\left(x, 2^{j+1+\frac{k}{2}} \sqrt{\delta^{\prime}}\right)}\left|\int_{\frac{t}{2}}^{t} e^{-(t-s) L} \operatorname{div}\left(1_{S_{\ell}\left(x, 2^{j+1+\frac{k}{2}} \sqrt{\delta^{\prime}}\right)} f(s, \cdot)\right)(y) \mathrm{d} s\right|^{2} \mathrm{~d} y \mathrm{~d} t\right)^{\frac{1}{2}} .
\end{aligned}
$$

For $\ell=1$, and $t \in\left(\frac{\delta^{\prime}}{2}, \delta^{\prime}\right)$, we have that

$$
\left\|\int_{\frac{t}{2}}^{t} e^{-(t-s) L} \operatorname{div}\left(1_{S_{1}\left(x, 2^{\left.j+1+\frac{k}{2} \sqrt{\delta^{\prime}}\right)}\right.} f(s, \cdot)\right)(y) \mathrm{d} s\right\|_{2} \leq \int_{\frac{t}{2}}^{t} \frac{1}{\sqrt{t-s}}\left\|1_{B\left(x, 2^{\left.j+2+\frac{k}{2} \sqrt{\delta^{\prime}}\right)}\right.} f(s, \cdot)\right\|_{2} \mathrm{~d} s,
$$

and thus

$$
\begin{aligned}
& \left(f_{\frac{\delta^{\prime}}{2}}^{\delta^{\prime}} f_{B\left(x, 2^{\left.j+1+\frac{k}{2} \sqrt{\delta^{\prime}}\right)}\right.}\left|\int_{\frac{t}{2}}^{t} e^{-(t-s) L} \operatorname{div}\left(1_{S_{1}\left(x, 2^{\left.j+1+\frac{k}{2} \sqrt{\delta^{\prime}}\right)}\right.} f(s, \cdot)\right)(y) \mathrm{d} s\right|^{2} \mathrm{~d} y \mathrm{~d} t\right)^{\frac{1}{2}} \\
& \quad \lesssim\left(\int_{\frac{\delta^{\prime}}{2}}^{\delta^{\prime}}\left(\int_{\frac{t}{2}}^{t} \frac{1}{\sqrt{\delta^{\prime}}} \frac{1}{\sqrt{t-s}} \|\left(2^{\left.j+1+\frac{k}{2} \sqrt{\delta^{\prime}}\right)^{-\frac{n}{2}}} \mathbb{l}_{B\left(x, 2^{\left.j+2+\frac{k}{2} \sqrt{\delta^{\prime}}\right)}\right.} f(s, \cdot) \|_{2} \mathrm{~d} s\right)^{2} \mathrm{~d} t\right)^{\frac{1}{2}} .\right.
\end{aligned}
$$


By Schur's Lemma, we thus have that

$$
\begin{aligned}
\left(f_{\frac{\delta^{\prime}}{2}}^{\delta^{\prime}}\right. & \left.f_{B\left(x, 2^{\left.j+1+\frac{k}{2} \sqrt{\delta^{\prime}}\right)} \mid\right.}\left|\int_{\frac{t}{2}}^{t} e^{-(t-s) L} \operatorname{div}\left(1_{S_{1}\left(x, 2^{j+1+\frac{k}{2} \sqrt{\delta^{\prime}}}\right)} f(s, \cdot)\right)(y) \mathrm{d} s\right|^{2} \mathrm{~d} y \mathrm{~d} t\right)^{\frac{1}{2}} \\
& \lesssim\left(\int_{\frac{\delta^{\prime}}{2}}^{\delta^{\prime}} f_{B\left(x, 2^{\left.j+2+\frac{k}{2} \sqrt{\delta^{\prime}}\right)}\right.}|f(t, y)|^{2} \mathrm{~d} y \mathrm{~d} t\right)^{\frac{1}{2}} \leq\left(\int_{0}^{\infty} f_{B\left(x, 2^{\left.j+3+\frac{k}{2} \sqrt{t}\right)}\right.}|f(t, y)|^{2} \mathrm{~d} y \mathrm{~d} t\right)^{\frac{1}{2}} .
\end{aligned}
$$

Let us now consider $\ell \geq 2$. We have that

$$
\begin{aligned}
\left(f_{\frac{\delta^{\prime}}{2}}^{\delta^{\prime}}\right. & \left.f_{B\left(x, 2^{\left.j+1+\frac{k}{2} \sqrt{\delta^{\prime}}\right)} \mid\right.}\left|\int_{\frac{t}{2}}^{t} e^{-(t-s) L} \operatorname{div}\left(1_{S_{\ell}\left(x, 2^{j+1+\frac{k}{2} \sqrt{\delta^{\prime}}}\right)} f(s, \cdot)\right)(y) \mathrm{d} s\right|^{2} \mathrm{~d} y \mathrm{~d} t\right)^{\frac{1}{2}} \\
& \lesssim\left(\int_{\frac{\delta^{\prime}}{2}}^{\delta^{\prime}}\left(\int_{\frac{t}{2}}^{t} \frac{1}{\sqrt{\delta^{\prime}}} \frac{1}{\sqrt{t-s}} 2^{\ell n} e^{-c \frac{\ell \ell+j_{2} \delta^{\prime}}{t-s}}\left(f_{B\left(x, 2^{\left.j+\ell+2+\frac{k}{2} \sqrt{\delta^{\prime}}\right)}\right.}|f(s, y)|^{2} \mathrm{~d} y\right)^{\frac{1}{2}} \mathrm{~d} s\right)^{2} \mathrm{~d} t\right)^{\frac{1}{2}} \\
& \lesssim 2^{\frac{\ell n}{2}} e^{-\frac{c}{2} 4^{\ell+j} 2^{k}}\left(\int_{\frac{\delta^{\prime}}{4}}^{\delta^{\prime}} f_{B\left(x, 2^{\left.j+\ell+2+\frac{k}{2} \sqrt{\delta^{\prime}}\right)}\right.}|f(s, y)|^{2} \mathrm{~d} y \mathrm{~d} s\right)^{\frac{1}{2}} .
\end{aligned}
$$

For $p=\infty$, summing in $j, k, \ell$, and using the change of angle lemma [6] in $T^{p, 2}$, we have that

$$
\begin{aligned}
\left\|\mathcal{R}_{L} f\right\|_{X^{p}} & \lesssim \sum_{j, k, \ell} 2^{\frac{(j+\ell) n}{2}} e^{-\frac{c}{2} 4^{\ell+j} 2^{k}}\left\|x \mapsto\left(\int_{0}^{\infty} f_{B\left(x, 2^{\left.j+2+\ell+\frac{k}{2} \sqrt{s}\right)}\right.}|f(s, y)|^{2} \mathrm{~d} y \mathrm{~d} s\right)^{\frac{1}{2}}\right\|_{L^{p}} \\
& \lesssim \sum_{j, k, \ell} 2^{\frac{(j+\ell) n}{2}} e^{-\frac{c}{2} 4^{\ell+j} 2^{k}} 2^{\left(j+\ell+\frac{k}{2}\right) \tau}\|f\|_{T^{p, 2}} \lesssim\|f\|_{T^{p, 2}},
\end{aligned}
$$

the number $\tau$ depending on $n$ and $p$. This suffices to sum. For $p=\infty$, we argue similarly. We note that the proof applies directly to any $f \in T^{\infty, 2}$ and gives a meaning to $\mathcal{R}_{L} f$.

The operators $\mathcal{R}_{L}$ and $\tilde{\mathcal{M}}_{L}$ are related in the following way.

Proposition 2.13. Let $p \in\left(p_{c}, \infty\right)$ as in Proposition 2.8 and $f \in T^{p, 2}$. Then $\nabla \mathcal{R}_{L} f \in T^{p, 2}$ and $\nabla \mathcal{R}_{L} f=\tilde{\mathcal{M}}_{L} f$ in $T^{p, 2}$.

Proof. Given Propositions 2.8 and 2.12, we only have to show that, for $f \in \mathscr{D}, \nabla \mathcal{R}_{L} f=$ $\tilde{\mathcal{M}}_{L} f$ in $\mathscr{D}^{\prime}$. Let $g \in \mathscr{D}$. As in the proof of Lemma 2.6, we have that (where $\langle\cdot, \cdot\rangle$ is the $L^{2}$ inner product)

$$
\begin{aligned}
\int_{\mathbb{R}}\left\langle\tilde{\mathcal{M}}_{L} f(t, \cdot), g(t, \cdot)\right\rangle \mathrm{d} t & =\int_{\mathbb{R}} \int_{\mathbb{R}} \mathbb{1}_{(0, \infty)}(t-s)\left\langle\nabla e^{-(t-s) L} \operatorname{div} f(s, \cdot), g(t, \cdot)\right\rangle \mathrm{d} s \mathrm{~d} t \\
& =-\int_{\mathbb{R}} \int_{\mathbb{R}} \mathbb{1}_{(0, \infty)}(t-s)\left\langle e^{-(t-s) L} \operatorname{div} f(s, \cdot), \operatorname{div} g(t, \cdot)\right\rangle \mathrm{d} s \mathrm{~d} t \\
& =-\int_{\mathbb{R}}\left\langle\mathcal{R}_{L} f(t, \cdot), \operatorname{div} g(t, \cdot)\right\rangle \mathrm{d} t=\int_{\mathbb{R}}\left\langle\nabla \mathcal{R}_{L} f(t, \cdot), g(t, \cdot)\right\rangle \mathrm{d} t
\end{aligned}
$$

\section{$3 \quad L^{2}$-theory and energy solutions}

\subsection{The space $\dot{W}(0, \infty)$}

We start with a structural lemma about distributions $u \in \dot{W}(0, \infty)$. Note that it is not restricted to solutions of our problem. 
Lemma 3.1. For all $u \in \dot{W}(0, \infty)$ there exist a unique $v \in \dot{W}(0, \infty) \cap \mathscr{C}_{0}\left(L^{2}\left(\mathbb{R}^{n}\right)\right)$ and $c \in \mathbb{C}$ such that $u=v+c$. Moreover,

$$
\|v\|_{L^{\infty}\left(L^{2}\right)} \leq \sqrt{2\|u\|_{L^{2}\left(\dot{H}^{1}\right)}\left\|\partial_{t} u\right\|_{L^{2}\left(\dot{H}^{-1}\right)}} .
$$

Proof. Set $w=\partial_{t} u+\Delta u$, and let $g \in L^{2}\left(L^{2}\right)$ be such that $w=\operatorname{div} g$. Given $t \geq 0$, we denote by $\tau_{t} g$ the time translation of $g$ defined by $\tau_{t} g(s,)=.g(s+t,$.$) for all s>0$. We now set, for all $t \geq 0$,

$$
v(t)=-\int_{t}^{\infty} e^{(s-t) \Delta} w(s) \mathrm{d} s=-\int_{0}^{\infty} e^{s \Delta} \operatorname{div}\left(\tau_{t} g\right)(s) \mathrm{d} s,
$$

where the integral is defined weakly as shown below. Indeed, for $f \in L^{2}\left(\mathbb{R}^{n}\right)$ and $t \geq 0$, we have that

$$
\int_{0}^{\infty}\left|\left\langle\tau_{t} g(s), \nabla e^{s \Delta} f\right\rangle\right| \mathrm{d} s \leq\left\|\tau_{t} g\right\|_{L^{2}\left(L^{2}\right)}\left\|(s, x) \mapsto \nabla e^{s \Delta} f(x)\right\|_{L^{2}\left(L^{2}\right)} \leq \frac{1}{\sqrt{2}}\left\|\tau_{t} g\right\|_{L^{2}\left(L^{2}\right)}\|f\|_{L^{2}}
$$

where the last inequality follows from a simple Fourier multiplier estimate. The argument also gives

$$
\left\|v(t)-v\left(t^{\prime}\right)\right\|_{L^{2}} \leq \frac{1}{\sqrt{2}}\left\|\tau_{t} g-\tau_{t^{\prime}} g\right\|_{L^{2}\left(L^{2}\right)} \quad \forall t, t^{\prime}>0,
$$

and therefore $v \in \mathscr{C}\left([0, \infty) ; L^{2}\right)$ as well as $\lim _{t \rightarrow \infty}\|v(t)\|_{L^{2}}=0$ as $\left\|\tau_{t} g\right\|_{L^{2}\left(L^{2}\right)} \underset{\tau \rightarrow \infty}{\longrightarrow} 0$ for all $g \in L^{2}\left(L^{2}\right)$. We now prove that $u-v$ is equal to a constant. By Remark 2.7 we have that

$$
\begin{array}{ll} 
& \|\nabla v\|_{L^{2}\left(L^{2}\right)}=\left\|\tilde{\mathcal{M}}_{-\Delta}^{*} g\right\|_{L^{2}\left(L^{2}\right)} \lesssim\|g\|_{L^{2}\left(L^{2}\right)}, \\
\text { hence } & \|\Delta v\|_{L^{2}\left(\dot{H}^{-1}\right)} \leq\|\nabla v\|_{L^{2}\left(L^{2}\right)} \lesssim\|g\|_{L^{2}\left(L^{2}\right)} .
\end{array}
$$

Moreover, $\partial_{t} v \in L^{2}\left(\dot{H}^{-1}\right)$ and $\partial_{t} v+\Delta v=w$ in $L^{2}\left(\dot{H}^{-1}\right)$. Indeed, for all $\phi \in \mathscr{D}$ we have that

$$
\begin{aligned}
\left\langle\partial_{t} v, \phi\right\rangle=-\left\langle v, \partial_{t} \phi\right\rangle & =\int_{0}^{\infty}\left\langle\int_{t}^{\infty} e^{(s-t) \Delta} w(s) \mathrm{d} s, \partial_{t} \phi(t)\right\rangle \mathrm{d} t \\
& =\int_{0}^{\infty}\left\langle w(s), \int_{0}^{s} e^{(s-t) \Delta} \partial_{t} \phi(t) \mathrm{d} t\right\rangle \mathrm{d} s \\
& =\int_{0}^{\infty}\left\langle w(s), \int_{0}^{s}\left[\partial_{t}\left(e^{(s-t) \Delta} \phi(t)\right)+e^{(s-t) \Delta} \Delta \phi(t)\right] \mathrm{d} t\right\rangle \mathrm{d} s \\
& =\langle w, \phi\rangle-\langle v, \Delta \phi\rangle .
\end{aligned}
$$

Consider the distribution $h:=u-v \in \dot{W}(0, \infty)$; we have that $\partial_{t} h+\Delta h=0$ in $L^{2}\left(\dot{H}^{-1}\right)$. Since $h \in L^{2}\left(\dot{H}^{1}\right)$, we have that $h \in L^{2}\left(\mathscr{S}^{\prime}\right)$. We can thus take the partial Fourier transform $\mathcal{F}_{x}$ in the $\mathbb{R}^{n}$ variable, and obtain that the distribution $\phi=\mathcal{F}_{x} h \in L^{2}\left(\mathscr{S}^{\prime}\right)$ satisfies

$$
\partial_{t} \phi-|\xi|^{2} \phi=0 \quad \text { in } \mathscr{D}^{\prime}
$$

where $m(t, \xi) T$ denotes the multiplication of $T \in \mathscr{D}^{\prime}$ by the function $m$, here the polynomial $(t, \xi) \mapsto|\xi|^{2}$. Solving the first order differential equation away from $\xi=0$, there exists $\alpha \in \mathscr{D}^{\prime}\left(\mathbb{R}^{n} \backslash\{0\}\right)$ such that

$$
\phi=e^{t|\xi|^{2}} \alpha \quad \text { in } \mathscr{D}^{\prime}\left((0, \infty) \times\left(\mathbb{R}^{n} \backslash\{0\}\right)\right) .
$$


Since $\xi \phi \in L^{2}\left(L^{2}\right)$ we have that $\xi \alpha e^{t|\xi|^{2}} \in L^{2}\left(L^{2}\left(\mathbb{R}^{n} \backslash\{0\}\right)\right)$. But for any compact set $K \subset \mathbb{R}^{n} \backslash\{0\}$, Fubini's theorem tells us that

$$
\int_{0}^{\infty} \int_{K}\left|\xi \alpha(\xi) e^{t|\xi|^{2}}\right|^{2} \mathrm{~d} \xi \mathrm{d} t=\infty
$$

unless $\alpha=0$ almost everywhere on $K$. Thus $\alpha=0$ in $\mathscr{D}^{\prime}\left(\mathbb{R}^{n} \backslash\{0\}\right)$. This implies that $\phi$ is supported in $(0, \infty) \times\{0\}$, and hence there exists $\tilde{c} \in \mathscr{D}^{\prime}(0, \infty)$ such that $\phi=\tilde{c} \otimes \delta_{0}$. But $\partial_{t} \phi \in L^{2}\left(\dot{H}^{-1}\right)$ so $\tilde{c}$ is constant. Taking the inverse partial Fourier transform, we have shown that there exists a constant $c \in \mathbb{C}$ such that $u=v+c$.

To prove uniqueness, let $v_{1}, v_{2} \in W(0, \infty)$ be such that there exists $c_{1}, c_{2} \in \mathbb{C}$ with $u=$ $v_{1}+c_{1}=v_{2}+c_{2}$ and define $w=v_{1}-v_{2}$. We have that $w \in \mathscr{C}_{0}\left(L^{2}\right)$ and $w=c_{2}-c_{1}$. Therefore, $w=0$, hence $c_{1}=c_{2}$ and the decomposition is unique.

We now prove the norm estimate. We have already shown that

$$
\begin{aligned}
\sup _{t \geq 0}\|v(t)\|_{L^{2}} & \leq \frac{1}{\sqrt{2}}\|w\|_{L^{2}\left(\dot{H}^{-1}\right)} \leq \frac{1}{\sqrt{2}}\left(\left\|\partial_{t} u\right\|_{L^{2}\left(\dot{H}^{-1}\right)}+\|\Delta u\|_{L^{2}\left(\dot{H}^{-1}\right)}\right) \\
& \leq \frac{1}{\sqrt{2}}\left(\left\|\partial_{t} u\right\|_{L^{2}\left(\dot{H}^{-1}\right)}+\|u\|_{L^{2}\left(\dot{H}^{1}\right)}\right) .
\end{aligned}
$$

We now apply the result to the scaled functions $u_{a}:(t, x) \mapsto a^{\frac{n}{2}} u(t, a x)$, and obtain that

$$
\sup _{t \geq 0}\|v(t)\|_{L^{2}} \leq \frac{1}{\sqrt{2}}\left(\frac{1}{a}\left\|\partial_{t} u\right\|_{L^{2}\left(\dot{H}^{-1}\right)}+a\|u\|_{L^{2}\left(\dot{H}^{1}\right)}\right),
$$

for all $a>0$. Optimising in $a$ gives that

$$
\sup _{t \geq 0}\|v(t)\|_{L^{2}} \leq \sqrt{2\left\|\partial_{t} u\right\|_{L^{2}\left(\dot{H}^{-1}\right)}\|u\|_{L^{2}\left(\dot{H}^{1}\right)}} .
$$

Remark 3.2. For each $u \in \dot{W}(0, \infty)$, the above lemma gives the existence of the limit $\lim _{t \rightarrow 0} u(t, \cdot)$ in $\mathscr{D}^{\prime}\left(\mathbb{R}^{n}\right)$, equal to $v(0)+c$. We call this limit the trace of $u$, and denote it by $\operatorname{Tr}(u)$.

Remark 3.3. It is a well-known fact that for $0 \leq a<b<\infty$, and $u, v \in \dot{W}(a, b) \cap$ $\mathscr{C}\left([a, b] ; L^{2}\right)$, we have that $t \mapsto\langle u(t), v(t)\rangle \in W^{1,1}(a, b)$ and

$$
\left(L^{2}\langle u(\cdot), v(\cdot)\rangle_{L^{2}}\right)^{\prime}={ }_{\dot{H}^{-1}}\left\langle u^{\prime}(\cdot), v(\cdot)\right\rangle_{\dot{H}^{1}}+{ }_{\dot{H}^{1}}\left\langle u(\cdot), v^{\prime}(\cdot)\right\rangle_{\dot{H}^{-1}} \in L^{1}(a, b)
$$

See, e.g., $[1, \S 14]$.

Remark 3.4. Lemma 3.1 is wrong if one replaces $\dot{W}(0, \infty)$ by $\dot{W}(a, b)$ for some finite $a<b$. To see this, take $f \in \dot{H}^{1}\left(\mathbb{R}^{n}\right) \backslash L^{2}\left(\mathbb{R}^{n}\right)$ and set $u(t, x)=f(x)$ for all $(t, x) \in(a, b) \times \mathbb{R}^{n}$.

\subsection{A priori energy estimates}

As a corollary of Lemma 3.1, we obtain the following a priori energy estimate.

Corollary 3.5. Let $u \in \mathscr{D}^{\prime}$ be a global weak solution of (1.1) such that $\nabla u \in L^{2}\left(L^{2}\right)$. Then there exists a constant $c \in \mathbb{C}$ such that $v:=u-c \in \mathscr{C}_{0}\left(L^{2}\right)$ and is norm decreasing, $\nabla v=\nabla u \in L^{2}\left(L^{2}\right), v$ is a weak solution of (1.1) and

$$
\|v(0)\|_{L^{2}}=\|v\|_{L^{\infty}\left(L^{2}\right)} \leq \sqrt{2 \Lambda}\|\nabla v\|_{L^{2}\left(L^{2}\right)} \leq \sqrt{\frac{\Lambda}{\lambda}}\|v(0)\|_{L^{2}},
$$

where $v(0)=v(0,$.$) , and \lambda, \Lambda$ are the ellipticity constants from (1.2). 
Proof. Since $\partial_{t} u=\operatorname{div} g$ in $\mathscr{D}^{\prime}$ for $g=A \nabla u \in L^{2}\left(L^{2}\right)$, we have that

$$
\left|\left\langle\partial_{t} u, \bar{\phi}\right\rangle\right| \leq \Lambda\|\nabla u\|_{L^{2}\left(L^{2}\right)}\|\nabla \phi\|_{L^{2}\left(L^{2}\right)},
$$

hence $\partial_{t} u \in L^{2}\left(\dot{H}^{-1}\right)$. Thus $u \in \dot{W}(0, \infty)$ and Lemma 3.1 imply that there exists a constant $c \in \mathbb{C}$ such that $v:=u-c \in \dot{W}(0, \infty) \cap \mathscr{C}_{0}\left(L^{2}\right)$, and

$$
\begin{aligned}
\|v\|_{L^{\infty}\left(L^{2}\right)} & \leq \sqrt{2\left\|\partial_{t} u\right\|_{L^{2}\left(\dot{H}^{-1}\right)}\|\nabla u\|_{L^{2}\left(L^{2}\right)}} \\
& \leq \sqrt{2\|g\|_{L^{2}\left(L^{2}\right)}\|\nabla u\|_{L^{2}\left(L^{2}\right)}} \leq \sqrt{2 \Lambda}\|\nabla u\|_{L^{2}\left(L^{2}\right)}=\sqrt{2 \Lambda}\|\nabla v\|_{L^{2}\left(L^{2}\right)} .
\end{aligned}
$$

Moreover, as constants are trivial weak solutions of (1.1), so is $v$. Let $b>a>0$. For all $U \in L^{2}\left(a, b ; \dot{H}^{1}\left(\mathbb{R}^{n}\right)\right)$, we have that

$$
\int_{a}^{b} \dot{H}^{-1}\left\langle\partial_{s} v(s, \cdot), U(s, \cdot)\right\rangle_{\dot{H}^{1}} \mathrm{~d} s=-\int_{a}^{b} \int_{\mathbb{R}^{n}} A(s, x) \nabla v(s, x) \cdot \overline{\nabla U(s, x)} \mathrm{d} x \mathrm{~d} s .
$$

For $U=v$, Remark 3.3 and ellipticity give that

$$
\begin{aligned}
\|v(a, \cdot)\|_{L^{2}}^{2}-\|v(b, \cdot)\|_{L^{2}}^{2} & =-2 \Re e \int_{a}^{b} \dot{H}^{-1}\left\langle\partial_{s} v(s, \cdot), v(s, \cdot)\right\rangle_{\dot{H}^{1}} \mathrm{~d} s \\
& =2 \Re e \int_{a}^{b} \int_{\mathbb{R}^{n}} A(s, x) \nabla v(s, x) \cdot \overline{\nabla v(s, x)} \mathrm{d} x \mathrm{~d} s \\
& \geq 2 \lambda\|\nabla v\|_{L^{2}\left(a, b ; L^{2}\right)}^{2}
\end{aligned}
$$

This gives the norm decreasing property and letting $a \rightarrow 0$ and $b \rightarrow \infty$, yields $2 \lambda\|\nabla v\|_{L^{2}\left(L^{2}\right)}^{2} \leq$ $\|v(0, \cdot)\|_{L^{2}}^{2}$. This completes the proof of Corollary 3.5.

These a priori estimates can be localised. This is well-known, but we include an argument for the convenience of the reader, and to record some explicit constants for later use.

Proposition 3.6. Let $(a, b) \subset(0, \infty), x \in \mathbb{R}^{n}, r>0$. Let $u \in L^{2}\left(a, b ; H^{1}(B(x, 2 r))\right)$ be $a$ local weak solution of $(1.1)$ on $(a, b) \times B(x, 2 r)$. Then $u \in \mathscr{C}\left([a, b] ; L^{2}(B(x, r))\right)$ and there exists $\kappa>0$ such that for all $c \in(a, b]$, we have

$$
\begin{aligned}
\|u(b, \cdot)\|_{L^{2}(B(x, r))}^{2} & \leq\left(\frac{4 \kappa^{2} \Lambda^{2}}{\lambda r^{2}}+\frac{1}{b-a}\right) \int_{a}^{b}\|u(s, \cdot)\|_{L^{2}(B(x, 2 r))}^{2} \mathrm{~d} t, \\
\int_{c}^{b}\|\nabla u(s, \cdot)\|_{L^{2}(B(x, r))}^{2} \mathrm{~d} s & \leq \frac{1}{\lambda(c-a)}\left(1+(b-a) \frac{4 \kappa^{2} \Lambda^{2}}{\lambda r^{2}}\right) \int_{a}^{b}\|u(s, \cdot)\|_{L^{2}(B(x, 2 r))}^{2} \mathrm{~d} s .
\end{aligned}
$$

Proof. Let $\eta \in \mathscr{C}_{c}^{\infty}\left(\mathbb{R}^{n}\right)$ be a real-valued function supported in $B(x, 2 r)$, such that $\eta(y)=1$ for all $y \in B(x, r),\|\eta\|_{\infty} \leq 1$, and $\|\nabla \eta\|_{\infty} \leq \frac{\kappa}{r}$. We have that

$$
\|\nabla(\eta u)\|_{L^{2}\left((a, b) \times \mathbb{R}^{n}\right)} \leq \frac{2 \kappa}{r}\|u\|_{L^{2}((a, b) \times B(x, 2 r))}+\|\nabla u\|_{L^{2}((a, b) \times B(x, 2 r))}<\infty .
$$

Therefore, $\eta u \in L^{2}\left((a, b), H_{0}^{1}(B(x, 2 r))\right)$. Note that this space is the closure of $\mathscr{C}_{c}^{\infty}((a, b) \times$ $B(x, 2 r))$ in $L^{2}\left((a, b), H^{1}(B(x, 2 r))\right)$. Let $\phi \in \mathscr{C}_{c}^{\infty}((a, b) \times B(x, 2 r))$. Since $u$ is a local weak solution, we have

$$
\left|\iint_{(a, b) \times B(x, 2 r)} u(t, y) \overline{\partial_{t} \phi(t, y)} \mathrm{d} y \mathrm{~d} t\right| \leq \Lambda\|\nabla u\|_{L^{2}((a, b) \times B(x, 2 r))}\|\nabla \phi\|_{L^{2}((a, b) \times B(x, 2 r))} .
$$


Using the known duality between $H_{0}^{1}(\Omega)$ and $H^{-1}(\Omega)$ for any open subset $\Omega$ of $\mathbb{R}^{n}$, this shows that $\partial_{t} u \in L^{2}\left((a, b), H^{-1}(B(x, 2 r))\right)$ and the same holds for $\partial_{t}(\eta u)$. Moreover, the integral on the left is $-L^{2}\left(a, b ; H^{-1}(B(x, 2 r))\right)\left\langle\partial_{t} u, \phi\right\rangle_{L^{2}\left(a, b ; H_{0}^{1}(B(x, 2 r))\right)}$. By Lions' result [30, Proposition 3.1], $\eta u \in \mathscr{C}\left([a, b] ; L^{2}(B(x, 2 r))\right.$ ) (see also [16, Theorem 1, Chapter XVIII]). Calculating for all $a^{\prime} \in(a, b)$ :

$$
\begin{aligned}
& \|\eta u(b, \cdot)\|_{L^{2}}^{2}-\left\|\eta u\left(a^{\prime}, \cdot\right)\right\|_{L^{2}}^{2}=2 \Re e \int_{a^{\prime}}^{b} H^{-1}(B(x, 2 r)) \\
= & \left.2 \Re e \int_{a^{\prime}}^{b} \partial_{t}(\eta u)(t, \cdot), \eta u(t, \cdot)\right\rangle_{H_{0}^{1}(B(x, 2 r))} \mathrm{d} t \\
= & -2 \Re e \int_{a^{\prime}}^{b} \int_{B(x, 2 r)} \eta(y) A(t, y) \nabla u(t, y) \cdot \eta(y) \overline{\nabla u(t, y)} \mathrm{d} y \mathrm{~d} t \\
& +4 \Re e \int_{a^{\prime}}^{b} \int_{B(x, 2 r)} \eta(y) A(t, y) \nabla u(t, y) \cdot \overline{u(t, y)} \nabla \eta(y) \mathrm{d} y \mathrm{~d} t .
\end{aligned}
$$

Therefore,

$$
\begin{aligned}
& \|\eta u(b, \cdot)\|_{L^{2}}^{2}+2 \lambda \int_{a^{\prime}}^{b}\|\eta \nabla u(s, \cdot)\|_{L^{2}}^{2} \mathrm{~d} s \\
& \quad \leq\left\|\eta u\left(a^{\prime}, \cdot\right)\right\|_{L^{2}}^{2}+\lambda \int_{a^{\prime}}^{b}\left(\|\eta \nabla u(s, \cdot)\|_{2}^{2}+\frac{4 \kappa^{2} \Lambda^{2}}{\lambda r^{2}}\|u(s, \cdot)\|_{L^{2}(B(x, 2 r))}^{2}\right) \mathrm{d} s,
\end{aligned}
$$

and thus

$$
\|\eta u(b, \cdot)\|_{2}^{2}+\lambda \int_{a^{\prime}}^{b}\|\eta \nabla u(s, \cdot)\|_{2}^{2} \mathrm{~d} s \leq\left\|\eta u\left(a^{\prime}, \cdot\right)\right\|_{2}^{2}+\int_{a^{\prime}}^{b} \frac{4 \kappa^{2} \Lambda^{2}}{\lambda r^{2}}\|u(s, \cdot)\|_{L^{2}(B(x, 2 r))}^{2} \mathrm{~d} s .
$$

Integrating in $a^{\prime}$ between $a$ and $b$ gives the inequalities:

$$
\begin{aligned}
\|u(b, \cdot)\|_{L^{2}(B(x, r))}^{2} & \leq\left(\frac{1}{b-a}+\frac{4 \kappa^{2} \Lambda^{2}}{\lambda r^{2}}\right) \int_{a}^{b}\|u(s, \cdot)\|_{L^{2}(B(x, 2 r))}^{2} \mathrm{~d} s \\
\lambda(c-a) \int_{c}^{b}\|\nabla u(s, \cdot)\|_{L^{2}(B(x, r))}^{2} \mathrm{~d} s & \leq \lambda \int_{a}^{b}(s-a)\|\nabla u(s, \cdot)\|_{L^{2}(B(x, r))}^{2} \mathrm{~d} s \\
& \leq\left(1+(b-a) \frac{4 \kappa^{2} \Lambda^{2}}{\lambda r^{2}}\right) \int_{a}^{b}\|u(s, \cdot)\|_{L^{2}(B(x, 2 r))}^{2} \mathrm{~d} s .
\end{aligned}
$$

Remark 3.7. The above proof shows that whenever $u$ is a weak solution on $(a, b) \times \Omega$ with $u \in L^{2}\left(a, b ; H^{1}(\Omega)\right)$ then $\partial_{t} u \in L^{2}\left(a, b ; H^{-1}(\Omega)\right)$. One can thus take any $\varphi \in$ $L^{2}\left(a, b ; H_{0}^{1}(\Omega)\right)$ as a test function in (1.1) and the integral $\iint u \overline{\partial_{t} \varphi}$ can be reinterpreted as $-\int\left\langle\partial_{t} u(t, \cdot), \varphi(t, \cdot)\right\rangle \mathrm{d} t$, where the brackets correspond to the $H^{-1}(\Omega), H_{0}^{1}(\Omega)$ duality. Also $u \in \mathscr{C}\left([a, b] ; L^{2}\left(\Omega^{\prime}\right)\right)$ for any $\Omega^{\prime}$ with $\overline{\Omega^{\prime}} \subset \Omega$.

Similar estimates hold for the backward equation up to a time $T>0$ :

$$
\partial_{s} \phi(s, x)=-\operatorname{div} A(s, \cdot)^{*} \nabla \phi(s, x), \quad 0 \leq s \leq T, \quad x \in \mathbb{R}^{n} .
$$

Again a weak solution to this equation on $(a, b) \times \Omega$ is a function $\phi \in L_{\text {loc }}^{2}\left(a, b ; H_{\text {loc }}^{1}(\Omega)\right)$ such that for all $\psi \in \mathscr{C}_{c}^{\infty}((a, b) \times \Omega)$,

$$
-\int_{a}^{b} \int_{\Omega} \phi(s, x) \overline{\partial_{s} \psi(s, x)} \mathrm{d} x \mathrm{~d} s=\int_{a}^{b} \int_{\Omega} A(s, x)^{*} \nabla \phi(s, x) \cdot \overline{\nabla \psi(s, x)} \mathrm{d} x \mathrm{~d} s .
$$


Lemma 3.8. Let $\phi$ be a weak solution of (3.2) on $(0, T) \times \Omega$. Then $u:(t, x) \mapsto \phi(T-t, x)$ is a local weak solution on $(0, T) \times \Omega$ of (1.1) in which the matrices $A(t, x)$ are replaced by $A(T-t, x)^{*}, t \in[0, T], x \in \Omega$.

Proof. Let $\psi \in \mathscr{C}_{c}^{\infty}((0, T) \times \Omega)$. Then $\tilde{\psi}:(t, x) \mapsto \psi(T-t, x) \in \mathscr{C}_{c}^{\infty}((0, T) \times \Omega)$ and $\partial_{t} \tilde{\psi}(t, x)=-\left(\partial_{t} \psi\right)(T-t, x)$ for all $t \in[0, T]$ and all $x \in \Omega$. Therefore, we have

$$
\begin{aligned}
\int_{0}^{T} \int_{\Omega} u(t, x) \overline{\partial_{t} \psi(t, x)} \mathrm{d} x \mathrm{~d} t & =\int_{0}^{T} \int_{\Omega} \phi(T-t, x) \overline{\partial_{t} \psi(t, x)} \mathrm{d} x \mathrm{~d} t \\
& =-\int_{0}^{T} \int_{\Omega} \phi(s, x) \overline{\partial_{s} \tilde{\psi}(s, x)} \mathrm{d} x \mathrm{~d} s \\
& =\int_{0}^{T} \int_{\Omega} A(s, x)^{*} \nabla \phi(s, x) \cdot \overline{\nabla \tilde{\psi}(s, x)} \mathrm{d} x \mathrm{~d} s \\
& =\int_{0}^{T} \int_{\Omega} A(T-t, x)^{*} \nabla u(t, x) \cdot \overline{\nabla \psi(t, x)} \mathrm{d} x \mathrm{~d} t
\end{aligned}
$$

where we have made the change of variable $s:=T-t$ twice and we have used (3.3).

Proposition 3.9. Let $\phi \in L^{2}\left(a, b ; H^{1}(B(x, 2 r))\right)$ be a weak solution of $(3.2)$ on $(a, b) \times$ $B(x, 2 r)$. Then $\phi \in \mathscr{C}\left([a, b] ; L^{2}(B(x, r))\right)$ and there exists $\kappa>0$ such that for all $d \in[a, b)$, we have

$$
\begin{aligned}
\|\phi(a, .)\|_{L^{2}(B(x, r))}^{2} & \leq\left(\frac{4 \kappa^{2}}{\lambda r^{2}}+\frac{1}{b-a}\right) \int_{a}^{b}\|\phi(s, \cdot)\|_{L^{2}(B(x, 2 r))}^{2}, \mathrm{~d} s \\
\int_{a}^{d}\|\nabla \phi(s, \cdot)\|_{L^{2}(B(x, r))}^{2} \mathrm{~d} s & \leq \frac{1}{\lambda(b-d)}\left(1+(b-a) \frac{4 \kappa^{2} \Lambda^{2}}{\lambda r^{2}}\right) \int_{a}^{b}\|\phi(s, \cdot)\|_{L^{2}(B(x, 2 r))}^{2} \mathrm{~d} s .
\end{aligned}
$$

Proof. Thanks to Lemma 3.8, we may apply the result of Proposition 3.6 to $u(t, x):=$ $\phi(a+b-t, x)$ for $t \in(a, b), x \in \mathbb{R}^{n}$ and $c:=a+b-d \in(a, b]$.

\subsection{Well-posedness of energy solutions}

Definition 3.10. Let $u_{0} \in L^{2}\left(\mathbb{R}^{n}\right)$. The problem

$$
\partial_{t} u=\operatorname{div} A \nabla u, \quad u \in \dot{W}(0, \infty), \quad \operatorname{Tr}(u)=u_{0}
$$

is said to be well-posed if there exists a unique $u \in \dot{W}(0, \infty)$ global weak solution of (1.1) such that $\operatorname{Tr}(u)=u_{0}$.

Theorem 3.11. For all $u_{0} \in L^{2}\left(\mathbb{R}^{n}\right)$, the problem

$$
\partial_{t} u=\operatorname{div} A \nabla u, \quad u \in \dot{W}(0, \infty), \quad \operatorname{Tr}(u)=u_{0}
$$

is well-posed. Moreover, $u \in \mathscr{C}_{0}\left([0, \infty) ; L^{2}\right),\|u(t, \cdot)\|_{L^{2}}$ is non increasing and

$$
\left\|u_{0}\right\|_{L^{2}}=\|u\|_{L^{\infty}\left(L^{2}\right)} \leq \sqrt{2 \Lambda}\|\nabla u\|_{L^{2}\left(L^{2}\right)} \leq \sqrt{\frac{\Lambda}{\lambda}}\left\|u_{0}\right\|_{L^{2}} .
$$


With some care because we are dealing with an unbounded time interval, it is possible to adapt the proof of Lions [30, Theorem 5.1] for the existence in order to construct a solution in $W(0, \infty)$. Nevertheless, we give a constructive approach to the $L^{2}$-existence theory, that plays a key role in the $L^{p}$ theory developed in later sections. The approach relies on approximations of $A$ and on taking weak* limits of the corresponding sequences of approximate solutions. We thus need the following lemma.

Lemma 3.12. Let $A_{k} \in L^{\infty}\left((0, \infty) ; L^{\infty}\left(\mathbb{R}^{n} ; \mathscr{M}_{n}(\mathbb{C})\right)\right)$ for $k \in \mathbb{N}$ be such that $(1.2)$ holds uniformly in $k$ and

$$
A_{k}(t, x) \underset{k \rightarrow \infty}{\longrightarrow} A(t, x) \quad \text { for almost every }(t, x) \in(0, \infty) \times \mathbb{R}^{n} .
$$

Let $u_{k}$ be a global weak solution of $\partial_{t} u=\operatorname{div} A_{k} \nabla u$ for all $k \in \mathbb{N}$, and assume that

$$
\sup _{k \in \mathbb{N}}\left(\left\|u_{k}\right\|_{L^{\infty}\left(L^{2}\right)}+\left\|\nabla u_{k}\right\|_{L^{2}}\right)<\infty .
$$

Then there exists a subsequence $\left(u_{k_{j}}\right)_{j \in \mathbb{N}}$ such that $\left(u_{k_{j}}\right)_{j \in \mathbb{N}}$ weak $k^{*}$ converges to $u$ in $L^{\infty}\left(L^{2}\right)$ and $\left(\nabla u_{k_{j}}\right)_{j \in \mathbb{N}}$ weak $k^{*}$ converges in $L^{2}\left(L^{2}\right)$. The limit $u \in L^{\infty}\left(L^{2}\right)$ is then a global weak solution of (1.1) such that $\nabla u \in L^{2}\left(L^{2}\right)$.

Proof. Let $k \in \mathbb{N}$. Note first that $u_{k} \in \dot{W}(0, \infty)$ since it is a weak solution of $\partial_{t} u=\operatorname{div} A_{k} \nabla u$ such that $\nabla u_{k} \in L^{2}\left(L^{2}\right)$. Since $u_{k} \in L^{\infty}\left(L^{2}\right)$, Lemma 3.1 gives us that $u_{k} \in \mathscr{C}_{0}\left(L^{2}\right)$. Therefore $\left(u_{k}(0, \cdot)\right)_{k \in \mathbb{N}}$ is uniformly bounded in $L^{2}\left(\mathbb{R}^{n}\right)$. Moreover $\left(\nabla u_{k}\right)_{k \in \mathbb{N}}$ is uniformly bounded in $L^{2}\left(L^{2}\right)$. We can thus extract a subsequence $\left(u_{k_{j}}\right)_{j \in \mathbb{N}}$ using Banach-Alaoglu's theorem for which there exists $u \in L^{\infty}\left(L^{2}\right)$ and $u_{0} \in L^{2}\left(\mathbb{R}^{n}\right)$ with

$$
\begin{array}{rll}
u_{k_{j}} & \underset{j \rightarrow \infty}{\longrightarrow} u & \text { weak* }^{*} \text { in } L^{\infty}\left(L^{2}\right), \\
\nabla u_{k_{j}} & \underset{j \rightarrow \infty}{\longrightarrow} \nabla u & \text { weak }^{*} \text { in } L^{2}\left(L^{2}\right), \\
u_{k_{j}}(0, \cdot) & \underset{j \rightarrow \infty}{\longrightarrow} u_{0} & \text { weak }^{*} \text { in } L^{2} .
\end{array}
$$

For all $\phi \in \mathscr{D}\left(\mathbb{R}^{n}\right)$ and all $t \geq 0$, Remark 3.3 and the fact that $u_{k} \in \mathscr{C}_{0}\left(L^{2}\right)$ for all $k \in \mathbb{N}$ give that

$$
\int_{\mathbb{R}^{n}} u_{k_{j}}(t, y) \overline{\phi(y)} \mathrm{d} y=\int_{\mathbb{R}^{n}} u_{k_{j}}(0, y) \overline{\phi(y)} \mathrm{d} y-\int_{0}^{t} \int_{\mathbb{R}^{n}} A_{k_{j}}(s, y) \nabla u_{k_{j}}(s, y) \cdot \overline{\nabla \phi(y)} \mathrm{d} y \mathrm{~d} s .
$$

Since the right hand side converges to $\int_{\mathbb{R}^{n}} u_{0}(y) \overline{\phi(y)} \mathrm{d} y-\int_{0}^{t} \int_{\mathbb{R}^{n}} A(s, y) \nabla u(s, y) \cdot \overline{\nabla \phi(y)} \mathrm{d} y \mathrm{~d} s$, the left hand side converges and its limit is equal to $\int_{\mathbb{R}^{n}} u(t, y) \overline{\phi(y)} \mathrm{d} y$ for almost every $t>0$. Modifying $u$ for almost no $t>0$, we can assume that the equality holds everywhere. Differentiating in $t$ proves that $\partial_{t} u(t, \cdot)=\operatorname{div} A(t, \cdot) \nabla u(t, \cdot)$ in $\dot{H}^{-1}$ for almost every $t>0$. Therefore $\partial_{t} u=\operatorname{div} A \nabla u$ in $L^{2}\left(\dot{H}^{-1}\right)$ and thus $u$ is a weak solution of (1.1).

Remark 3.13. It is even possible to show strong convergence if $u_{k}(0, \cdot)$ are independent of $k$.

Proof of Theorem 3.11. We start with the proof of existence of a solution $u \in W(0, \infty)$ satisfying (1.1) and $u(0, \cdot)=u_{0}$. 
Step 0: We first consider $A$ independent of $t$. We let $L=-\operatorname{div} A \nabla$ and $u(t)=e^{-t L} u_{0}$. From semigroup theory, we know that $u \in \mathscr{C}_{0}\left([0, \infty) ; L^{2}\left(\mathbb{R}^{n}\right)\right) \cap \mathscr{C}^{\infty}(0, \infty ; D(L))$ is a (strong) solution of $\partial_{t} u+L u=0$. Moreover, $\nabla u \in L^{2}\left(L^{2}\right)$ and

$$
2 \lambda\|\nabla u\|_{L^{2}\left(L^{2}\right)}^{2} \leq 2 \Re e \int_{0}^{\infty} L^{2}\langle A \nabla u(t), \nabla u(t)\rangle_{L^{2}} \mathrm{~d} t=-\int_{0}^{\infty}\left(\|u(t)\|_{L^{2}}^{2}\right)^{\prime} \mathrm{d} t=\left\|u_{0}\right\|_{L^{2}}^{2} .
$$

Finally, one easily checks that $u$ is a global weak solution as well.

Step 1: We next consider $A$ of the form

$$
A(t, x)=\sum_{k=0}^{N} \mathbb{1}_{\left[t_{k}, t_{k+1}\right)}(t) A_{k}(x)+\mathbb{1}_{\left[t_{N+1},+\infty\right.}(t) A_{N+1}(x)
$$

for some $N \in \mathbb{N},\left(t_{k}\right)_{0 \leq k \leq N+1}$ an increasing sequence in $[0, \infty)$ with $t_{0}=0$ and $\left(A_{k}\right)_{0 \leq k \leq N+1}$ satisfying (1.2) uniformly. It is convenient to set $t_{N+2}=\infty$. For $j=0, \ldots, N+1$, let $L_{j}=-\operatorname{div} A_{j} \nabla$ and define

$$
\Gamma_{A}(t, s):=e^{-\left(t-t_{j}\right) L_{j}} e^{-\left(t_{j}-t_{j-1}\right) L_{j-1}} \ldots e^{-\left(t_{i+1}-s\right) L_{i}}
$$

for $t \in\left[t_{j}, t_{j+1}\right)$ and $s \in\left[t_{i}, t_{i+1}\right)$. We define $u: t \mapsto \Gamma_{A}(t, 0) u_{0}, t \geq 0$. That $u \in$ $\mathscr{C}_{0}\left([0, \infty) ; L^{2}\left(\mathbb{R}^{n}\right)\right)$ is easily established using the properties of the semigroups $\left(e^{-t L_{j}}\right)_{t \geq 0}$. We proceed inductively on $k$ to check the desired properties on $u$. Since $-L_{0}$ generates an analytic semigroup of contractions

$$
\left\|(t, x) \mapsto \mathbb{1}_{\left(0, t_{1}\right)} \Gamma_{A}(t, 0) u_{0}(x)\right\|_{L^{\infty}\left(L^{2}\right)} \leq\left\|u_{0}\right\|_{L^{2}}
$$

Therefore

$$
\left\|(t, x) \mapsto \mathbb{1}_{\left(0, t_{1}\right)}(t) \nabla u(t, x)\right\|_{L^{2}\left(L^{2}\right)} \leq\left\|(t, x) \mapsto \mathbb{1}_{\left(0, t_{1}\right)}(t) \nabla e^{-t L_{0}} u_{0}(x)\right\|_{L^{2}\left(L^{2}\right)} \lesssim\left\|u_{0}\right\|_{L^{2}},
$$

Moreover $\partial_{t} u(t, \cdot) \in L^{2}\left(\mathbb{R}^{n}\right)$ for all $t \in\left(0, t_{1}\right)$ and $\partial_{t}(u(t, \cdot))=L_{0} u(t, \cdot)=L(t) u(t, \cdot)$ in $L^{2}\left(\mathbb{R}^{n}\right)$ for all $t \in\left(0, t_{1}\right)$. Now let $k \leq N+1$ and assume that the following holds:

$$
\begin{aligned}
& \left\|(t, x) \mapsto \mathbb{1}_{\left(0, t_{k}\right)}(t) \Gamma_{A}(t, 0) u_{0}(x)\right\|_{L^{\infty}\left(L^{2}\right)} \leq\left\|u_{0}\right\|_{L^{2}}, \\
& \left\|(t, x) \mapsto \mathbb{1}_{\left(0, t_{k}\right)}(t) \nabla \Gamma_{A}(t, 0) u_{0}(x)\right\|_{L^{2}\left(L^{2}\right)} \lesssim\left\|u_{0}\right\|_{L^{2}},
\end{aligned}
$$

and $\partial_{t} u(t, \cdot)=L(t) u(t, \cdot)$ in $L^{2}\left(\mathbb{R}^{n}\right)$ for all $t \in\left(0, t_{k}\right) \backslash\left\{t_{0}, \ldots, t_{k-1}\right\}$.

Here, the implicit constants may depend on $N$ but we are inducting on a finite number of steps and we will get the dependence only on the ellipticity constants in (1.2) eventually. We want to extend all this to $t_{k+1}$. For $t \in\left[t_{k}, t_{k+1}\right)$ we have that

$$
u(t, \cdot)=\Gamma_{A}(t, s) u(s, \cdot)=e^{-\left(t-t_{k}\right) L_{k}} e^{-\left(t_{k}-s\right) L_{k-1}} u(s, \cdot)
$$

for all $s \in\left(t_{k-1}, t_{k}\right)$. Therefore

$$
\left\|(t, x) \mapsto \mathbb{1}_{\left(0, t_{k+1}\right)}(t) u(t, x)\right\|_{L^{\infty}\left(L^{2}\right)} \leq\left\|(t, x) \mapsto \mathbb{1}_{\left(0, t_{k}\right)}(t) u(t, x)\right\|_{L^{\infty}\left(L^{2}\right)} \leq\left\|u_{0}\right\|_{L^{2}} .
$$

Using $u(t, \cdot)=e^{-\left(t-t_{k}\right) L_{k}} u\left(t_{k}, \cdot\right)$, we have

$$
\left\|(t, x) \mapsto \mathbb{1}_{\left(t_{k}, t_{k+1}\right)}(t) \nabla u(t, x)\right\|_{L^{2}\left(L^{2}\right)} \lesssim\left\|u\left(t_{k}, \cdot\right)\right\|_{L^{2}} \leq\left\|u_{0}\right\|_{L^{2}}
$$


We also have that $\partial_{t} u(t, \cdot)=-L_{k} u(t, \cdot)=-L(t) u(t, \cdot)$ in $L^{2}\left(\mathbb{R}^{n}\right)$ for all $t \in\left(t_{k}, t_{k+1}\right)$. This concludes the induction, which proves that $u \in L^{\infty}\left(L^{2}\right) \cap L^{2}\left(\dot{H}^{1}\right)$, and that $u$ satisfies

$$
\partial_{t} u(t, \cdot)=-L(t) u(t, \cdot) \quad \forall t \in(0, \infty) \backslash\left\{t_{k} ; k \in \mathbb{N}\right\} .
$$

We now show that $u$ is a global weak solution of (1.1). Let $\phi \in \mathscr{D}$, and pick $M>t_{N+1}$ such that $\operatorname{supp} \phi \subset(0, M) \times \mathbb{R}^{n}$. For $j=0, \ldots, N+1, t \mapsto\langle u(t, \cdot), \phi(t, \cdot)\rangle$ (where $\langle\cdot, \cdot\rangle$ denotes the $L^{2}$ duality) is $\mathscr{C}^{1}$ on $\left(t_{j}, t_{j+1}\right)$ and continuous on $\left[t_{j}, t_{j+1}\right]$, hence

$\int_{t_{j}}^{t_{j+1}}\left\langle u(t, \cdot), \partial_{t} \phi(t, \cdot)\right\rangle \mathrm{d} t=\left\langle u\left(t_{j+1}, \cdot\right), \phi\left(t_{j+1}, \cdot\right)\right\rangle-\left\langle u\left(t_{j}, \cdot\right), \phi\left(t_{j}, \cdot\right)\right\rangle+\int_{t_{j}}^{t_{j+1}}\left\langle L_{j} u(t,),. \phi(t,).\right\rangle \mathrm{d} t$.

Summing in $j$ and using $\left\langle L_{j} u(t, \cdot), \phi(t)\right\rangle=-\left\langle A_{j} \nabla u(t, \cdot), \nabla \phi(t)\right\rangle$ for all $t \in\left(t_{j}, t_{j+1}\right)$, and the fact that $\operatorname{supp} \phi \subset(0, M) \times \mathbb{R}^{n}$, we have that

$$
\int_{0}^{\infty} \int_{\mathbb{R}^{n}} u(t, y) \overline{\partial_{t} \phi(t, y)} \mathrm{d} y \mathrm{~d} t=\int_{0}^{\infty} \int_{\mathbb{R}^{n}} A(t, y) \nabla u(t, y) \cdot \overline{\nabla \phi(t, y)} \mathrm{d} y \mathrm{~d} t
$$

i.e. $u$ is a weak solution of (1.1).

Therefore, by Corollary 3.5, $\|\nabla u\|_{L^{2}\left(L^{2}\right)} \sim\left\|u_{0}\right\|_{L^{2}}$ with constants depending only on $\lambda$ and $\Lambda$ from (1.2).

Step 2: We now consider $A$ of the form

$$
A:(t, x) \mapsto \sum_{k=0}^{\infty} \mathbb{1}_{\left[t_{k}, t_{k+1}\right)}(t) A_{k}(x)
$$

for some increasing sequence $\left(t_{k}\right)_{k \in \mathbb{N}}$ with $t_{0}=0$ and $\lim _{k \rightarrow \infty} t_{k}=+\infty$ and $\left(A_{k}\right)_{t \in \mathbb{N}}$ satisfying (1.2) uniformly. Define

$$
\mathcal{A}_{N}:(t, x) \mapsto \sum_{k=0}^{N} \mathbb{1}_{\left[t_{k}, t_{k+1}\right)}(t) A_{k}(x)+\mathbb{1}_{\left[t_{N+1},+\infty\right)}(t) A_{N+1}(x)
$$

for all $N \in \mathbb{N}$. Then $\mathcal{A}_{N}(t, x) \underset{N \rightarrow \infty}{\longrightarrow} A(t, x)$ for almost every $(t, x) \in(0, \infty) \times \mathbb{R}^{n}$. Let $\left(u_{N}\right)_{N \in \mathbb{N}}$ be the corresponding sequence of weak solutions to $\partial_{t} u=\operatorname{div} \mathcal{A}_{N} \nabla u$ obtained in the previous step. By Lemma 3.12, there exists a subsequence $\left(u_{N_{j}}\right)_{j \in \mathbb{N}}$ converging to $u \in L^{\infty}\left(L^{2}\right)$ in the weak* topology, with $u$ a weak solution of $\partial_{t} u=\operatorname{div} A \nabla u$ and $\|u\|_{L^{\infty}\left(L^{2}\right)}+\|\nabla u\|_{L^{2}\left(L^{2}\right)} \lesssim\left\|u_{0}\right\|_{L^{2}}$ with constants depending only on the ellipticity constants.

Step 3: We now turn to the case where $A \in \mathscr{C}\left([0, \infty) ; L^{\infty}\left(\mathbb{R}^{n} ; \mathscr{M}_{n}(\mathbb{C})\right)\right)$. Approximating $A$ almost everywhere by matrices of the form $(t, x) \mapsto \sum_{k=0}^{\infty} 1_{\left[t_{k}, t_{k+1}\right)}(t) A_{k}(x)$, with $A_{k}=A\left(t_{k}, \cdot\right)$, which satisfy (1.2) uniformly in $k$, we obtain from Step 2 a family of weak solutions $\left(u_{j}\right)_{j \in \mathbb{N}}$, uniformly bounded in $L^{\infty}\left(L^{2}\right)$ and such that $\sup _{j \in \mathbb{N}}\left\|\nabla u_{j}\right\|_{L^{2}\left(L^{2}\right)}<\infty$. Using Lemma 3.12 again we obtain a weak solution $u$ of (1.1) such that $\|u\|_{L^{\infty}\left(L^{2}\right)}+\|\nabla u\|_{L^{2}\left(L^{2}\right)} \lesssim\left\|u_{0}\right\|_{L^{2}}$.

Step 4: Finally, for a general $A \in L^{\infty}\left((0, \infty) ; L^{\infty}\left(\mathbb{R}^{n} ; \mathscr{M}_{n}(\mathbb{C})\right)\right)$ we can use the approximations

$$
\left(\tilde{A}_{j}:(t, x) \mapsto j \int_{t}^{t+\frac{1}{j}} A(s, x) \mathrm{d} s\right) \in \mathscr{C}\left([0, \infty) ; L^{\infty}\left(\mathbb{R}^{n} ; \mathscr{M}_{n}(\mathbb{C})\right)\right),
$$


for $j \geq 1$ and use Step 3 together with Lemma 3.12 one more time.

Step 5: Let us now prove uniqueness of solutions. Let $u, v \in \dot{W}(0, \infty)$ be solutions of (1.1) with $\operatorname{Tr}(u)=\operatorname{Tr}(v)=u_{0}$. The function $w:=u-v \in \dot{W}(0, \infty)$ is a global weak solution of (1.1) such that $\operatorname{Tr}(w)=0$. By Corollary 3.5, we have that there exists $c \in \mathbb{C}$, and $\tilde{w} \in \mathscr{C}_{0}\left(L^{2}\right)$ such that $w=\tilde{w}+c$. Since $\operatorname{Tr}(w)=0$, we have that $c=0$ and $\lim _{t \rightarrow 0} \tilde{w}(t, \cdot)=0$ in $L^{2}\left(\mathbb{R}^{n}\right)$. Corollary 3.5 thus yields $\|w\|_{L^{\infty}\left(L^{2}\right)}=\|\tilde{w}\|_{L^{\infty}\left(L^{2}\right)}=0$.

\subsection{Propagators}

Lemma 3.14. There exists a family of contractions $\{\Gamma(t, s) ; 0 \leq s \leq t<\infty\} \subset \mathscr{L}\left(L^{2}\right)$ such that

(1) $\Gamma(t, t)=I \quad \forall t \geq 0$.

(2) $\Gamma(t, s) \Gamma(s, r)=\Gamma(t, r) \quad \forall t \geq s \geq r \geq 0$.

(3) For all $h \in L^{2}\left(\mathbb{R}^{n}\right)$, and $s \geq 0, t \mapsto \Gamma(t, s) h \in \mathscr{C}_{0}\left([s, \infty) ; L^{2}\left(\mathbb{R}^{n}\right)\right)$.

(4) For all $u_{0} \in L^{2}\left(\mathbb{R}^{n}\right),(t, x) \mapsto \Gamma(t, 0) u_{0}(x)$ is a global weak solution of (1.1).

Proof. Let $u_{0} \in L^{2}\left(\mathbb{R}^{n}\right)$. Let $u$ be the solution of the Cauchy problem in Theorem 3.11. We have $u \in \mathscr{C}_{0}\left(L^{2}\right) \cap L^{2}\left(\dot{H}^{1}\right)$, with $\|u(t, \cdot)\|_{L^{2}} \leq\left\|u_{0}\right\|_{L^{2}}$ and we define $\Gamma(t, 0)$ as the contraction on $L^{2}$ that maps $u_{0}$ to $u(t, \cdot)$. Similarly, we can start from any time $s \geq 0$ and from any data $h \in L^{2}$, and obtain a unique solution $v \in \dot{W}(s, \infty)$ with $u(s, \cdot)=h$. We define $\Gamma(t, s)$ as the operator mapping $h$ to $u(t, \cdot)$ when $t \geq s$. Then (1), (3) and (4) follow by construction, while (2) follows from uniqueness.

Definition 3.15. We call $\{\Gamma(t, s) ; 0 \leq s \leq t<\infty\}$ the family of propagators for (1.1).

The restriction $s \geq 0$ only comes from the fact that we work on $(0, \infty) \times \mathbb{R}^{n}$. This means that, provided that $A$ is defined on $\mathbb{R}^{n+1}$ and satisfies (1.2), one can define $\Gamma(t, s)$ for $-\infty<s \leq t<\infty$ and we have the same properties on the full range of $s$ and $t$. One works on $(s, \infty)$ for arbitrary $s$ and by uniqueness, any two families are consistent on the common time intervals. There is a similar family for the backward equation (3.2).

Lemma 3.16. Let $T>0$. There exists a family of contractions $\{\tilde{\Gamma}(t, T) ; t \in(-\infty, T]\} \subset$ $\mathscr{L}\left(L^{2}\right)$ such that

1. $\tilde{\Gamma}(T, T)=I$.

2. For all $h \in L^{2}\left(\mathbb{R}^{n}\right), t \mapsto \tilde{\Gamma}(t, T) h \in \mathscr{C}_{0}\left((-\infty, T] ; L^{2}\right)$.

3. For all $h \in L^{2}\left(\mathbb{R}^{n}\right),(t, x) \mapsto \tilde{\Gamma}(t, T) h(x)$ is a global weak solution of $(3.2)$ on $(-\infty, T)$.

Proof. Define

$$
\tilde{A}(s, x)=\left\{\begin{array}{l}
A^{*}(T-s, x) \quad \text { if }(s, x) \in(-\infty, T] \times \mathbb{R}^{n}, \\
A^{*}(0, x) \text { if }(s, x) \in(T, \infty) \times \mathbb{R}^{n} .
\end{array}\right.
$$

Applying Theorem 3.11 on $(0, \infty)$ with $A$ replaced by $\tilde{A}$ we get the conclusion of Lemma 3.14 . Denoting the corresponding family of propagators by $\{\underset{\sim}{\Gamma}(t, s) ; 0 \leq s \leq t<\infty\} \subset \mathscr{L}\left(L^{2}\right)$, we define

$$
\tilde{\Gamma}(t, T):=\underset{\sim}{\Gamma}(T-t, 0) \quad \forall t \in(-\infty, T] .
$$


It is immediate that $\tilde{\Gamma}$ satisfies points 1 and 2. By Lemma 3.8, we have that $(t, x) \mapsto$ $\tilde{\Gamma}(t, T) h(x)$ is a weak solution of $(3.2)$ on $(-\infty, T) \times \mathbb{R}^{n}$, which proves point 3 .

Proposition 3.17. Let $T>0$. The families of propagators for (1.1) and (3.2) (up to time T) are related by

$$
\tilde{\Gamma}(t, T)=\Gamma(T, t)^{*} \quad \forall t \in[0, T] .
$$

In particular, for all $h \in L^{2}\left(\mathbb{R}^{n}\right), t \mapsto \Gamma(T, t)^{*} h$ is strongly continuous from $[0, T]$ into $L^{2}\left(\mathbb{R}^{n}\right)$ and $t \mapsto \Gamma(T, t) h$ is weakly continuous from $[0, T]$ into $L^{2}\left(\mathbb{R}^{n}\right)$.

Proof. Let $g, h \in L^{2}\left(\mathbb{R}^{n}\right)$, and $0 \leq t \leq s \leq T$. Let $u(s, x)=\tilde{\Gamma}(s, T) h(x)=\underset{\sim}{\Gamma}(T-s, 0) h(x)$, and $v(s, x)=\Gamma(s, t) g(x)$ for all $(s, x) \in[t, T] \times \mathbb{R}^{n}$. Since $u, v \in \dot{W}(t, T)$, we have, for almost every $s \in[t, T]$,

$$
\begin{aligned}
\left\langle\partial_{s} u(s, \cdot), v(s, \cdot)\right\rangle-\left\langle A^{*}(s, \cdot) \nabla u(s, \cdot), \nabla v(s, \cdot)\right\rangle & =0, \\
\left\langle u(s, \cdot), \partial_{s} v(s, \cdot)\right\rangle+\langle\nabla u(s, \cdot), A(s, \cdot) \nabla v(s, \cdot)\rangle & =0 .
\end{aligned}
$$

We therefore have (see Remark 3.3):

$$
\begin{aligned}
0 & =\int_{t}^{T} \partial_{s}\langle u(s, .), v(s, .)\rangle \mathrm{d} s=\langle u(T, .), v(T, .)\rangle-\langle u(t, .), v(t, .)\rangle \\
& =\langle h, \Gamma(T, t) g\rangle-\langle\tilde{\Gamma}(t, T) h, g\rangle .
\end{aligned}
$$

Remark 3.18. The restriction $T>0$ is irrelevant in the previous results and is only made because we study $(1.1)$ on $(0, \infty)$. The adjoint formula is independent of the choice of the extension of $A^{*}(T-t, x)$ for $t>T$ to construct $\tilde{\Gamma}$ in Lemma 3.16. It follows from this adjoint formula that any result we obtain for (1.1) involving the propagators $\Gamma(t, s)$ has its counterpart for the adjoint backward equation (3.2) globally on $(-\infty, T)$ or locally on $(S, T)$, with the propagators $\Gamma(t, s)^{*}$, provided the hypotheses made on the coefficients are stable under taking adjoints.

A key property of $\Gamma$ is that it satisfies the following $L^{2}-L^{2}$ off-diagonal bounds.

Proposition 3.19. For all Borel sets $E, F \subset \mathbb{R}^{n}$, all $f \in L^{2}\left(\mathbb{R}^{n}\right)$ and all $0 \leq s<t<\infty$,

$$
\left\|\mathbb{1}_{E} \Gamma(t, s)\left(\mathbb{1}_{F} f\right)\right\|_{L^{2}} \leq e^{-\alpha \frac{d(E, F)^{2}}{t-s}}\left\|\mathbb{1}_{F} f\right\|_{L^{2}},
$$

with $\alpha=\frac{\lambda}{4 \Lambda^{2}}$, where $\Lambda, \lambda$ are the ellipticity constants from (1.2) and $d(E, F)$ denotes the Hausdorff distance between $E$ and $F$ with Euclidean norm.

Proof. This result is already in [2]. The simple proof with this constant is taken from [21]. There, $A$ was assumed to be smooth but this is not necessary. It also adapts to systems with Gårding inequality instead of pointwise lower bounds. We reproduce the argument for the convenience of the reader. It is enough to assume $s=0$ as one can translate the origin of time to $s$. Let $\psi$ be a non negative, Lipschitz and bounded function on $\mathbb{R}^{n}$ with $|\nabla \psi| \leq \gamma$. For $f \in L^{2}\left(\mathbb{R}^{n}\right)$, set $\Gamma^{\psi}(t, 0) f=e^{\psi} \Gamma(t, 0)\left(e^{-\psi} f\right) \in L^{2}\left(\mathbb{R}^{n}\right)$ as $\psi$ is bounded. Observe that $u(t)=e^{-\psi} \Gamma^{\psi}(t, 0) f=\Gamma(t, 0)\left(e^{-\psi} f\right)$ is a global energy solution of (1.1). Using Remark 3.3, 
we have the chain of equalities and inequalities for almost every $t>0$ :

$$
\begin{aligned}
\frac{\mathrm{d}}{\mathrm{d} t}\left\|\Gamma^{\psi}(t, 0) f\right\|_{L^{2}}^{2} & =\frac{\mathrm{d}}{\mathrm{d} t}\left\|e^{\psi} u(t)\right\|_{L^{2}}^{2} \\
& =2 \Re e_{\dot{H}^{-1}}\left\langle\partial_{t}\left(e^{\psi} u(t)\right), e^{\psi} u(t)\right\rangle_{\dot{H}^{1}} \\
& =2 \Re e_{\dot{H}^{-1}}\left\langle\partial_{t} u(t), e^{2 \psi} u(t)\right\rangle_{\dot{H}^{1}} \\
& =-2 \Re e_{L^{2}}\left\langle A(t) \nabla u(t), \nabla\left(e^{2 \psi} u(t)\right)\right\rangle_{L^{2}} \\
& =-2 \Re e_{L^{2}}\left\langle A(t) e^{\psi} \nabla u(t), e^{\psi} \nabla u(t)\right\rangle_{L^{2}}-4 \Re e_{L^{2}}\left\langle A(t) e^{\psi} \nabla u(t), e^{\psi} u(t) \nabla \psi\right\rangle_{L^{2}} \\
& \leq-2 \lambda\left\|e^{\psi} \nabla u(t)\right\|_{L^{2}}^{2}+4 \Lambda \gamma\left\|e^{\psi} \nabla u(t)\right\|_{L^{2}}\left\|e^{\psi} u(t)\right\|_{L^{2}} \\
& \leq \frac{2 \Lambda^{2}}{\lambda} \gamma^{2}\left\|e^{\psi} u(t)\right\|_{L^{2}}^{2} .
\end{aligned}
$$

As $\Gamma^{\psi}(t, 0) f \rightarrow f$ in $L^{2}\left(\mathbb{R}^{n}\right)$ as $t \rightarrow 0$, we get

$$
\left\|\Gamma^{\psi}(t, 0) f\right\|_{L^{2}} \leq e^{\kappa \gamma^{2} t}\|f\|_{L^{2}}, \quad \kappa=\frac{\Lambda^{2}}{\lambda} .
$$

Assume now that $\operatorname{supp} f \subset F$ and let $\psi(x)=\inf (\gamma d(x, F), N)$ for a large $N>\gamma d(E, F)$. We obtain

$$
\|\Gamma(t, 0) f\|_{L^{2}(E)} \leq e^{-\gamma d(E, F)}\left\|\Gamma^{\psi}(t, 0) f\right\|_{L^{2}} \leq e^{\kappa \gamma^{2} t-\gamma d(E, F)}\|f\|_{L^{2}} .
$$

Optimizing with $\gamma=\frac{d(E, F)}{2 \kappa t}$ completes the proof.

\subsection{Connection with earlier constructions}

Suppose we have constructed $\Gamma(t, s)$ for all $-\infty<s \leq t<\infty$ as explained above after Definition 3.15.

Proposition 3.20. Fix $T>0$, let $u_{0} \in L^{2}\left(\mathbb{R}^{n}\right)$ and $u(t, \cdot)=\Gamma(t, 0) u_{0}$ for $t \geq 0$. Then $u$ agrees with Aronson's energy solution on $(0, T) \times \mathbb{R}^{n}$ and with Lions' energy solution on $(0, T) \times \mathbb{R}^{n}$ of (1.1). In particular, for $0 \leq s \leq t \leq T, \Gamma(t, s)$ agrees with both Aronson's and Lions' propagators.

Proof. We begin with Lions's construction [30, Theorem 5.1] (see also [16, Chap. XVIII, §3]). He proves well-posedness of (1.1) in the class $W(0, T)$ with data $u_{0}$. By our construction, we have that $u \in W(0, \infty)$, hence its restriction to $(0, T)$ belongs to $W(0, T)$. Thus, $u$ agrees with Lions's energy solution on $(0, T)$.

We turn to Aronson's construction [2]. This particular part of his article does not use the specificity of real coefficients. He proves well-posedness in the class $A_{T}=L^{\infty}\left(0, T ; L^{2}\left(\mathbb{R}^{n}\right)\right) \cap$ $L^{2}\left(0, T ; H^{1}\left(\mathbb{R}^{n}\right)\right)$ with data $u_{0}$. By our construction, we have that $u \in W(0, \infty)$, hence its restriction to $(0, T)$ belongs to $A_{T}$. Thus, $u$ agrees with Aronson's energy solution on $(0, T)$.

The consequence for the propagator $\Gamma(t, 0)$ is immediate. A translation of the origin of time to $s$ proves the result for $\Gamma(t, s)$.

It follows from this lemma that our propagators are universal for any local in time problem. This is particularly noticeable for Aronson's work with real coefficients as he constructs the kernel of $\Gamma(t, s)$ by using approximations by the propagators obtained by the standard parametrix constructions for equations with smooth coefficients on bounded cylinders. Our approach is totally opposite as we construct the "largest" possible object and restrict it. It will be useful to have shown uniqueness in the largest possible energy class $\dot{W}(0, \infty)$ later on. 


\section{A priori estimates}

We first prove a priori estimates for arbitrary weak solutions. We then turn to solutions of the form $(t, x) \mapsto \Gamma(t, 0) f(x)$ for $f$ in an $L^{p}$ space.

\subsection{Reverse Hölder estimates and consequences}

We consider the parabolic quasi-distance on $(0, \infty) \times \mathbb{R}^{n}$ defined by

$$
d((t, x),(s, y))=\max \{\sqrt{|t-s|},|x-y|\}, \quad(t, x),(s, y) \in(0, \infty) \times \mathbb{R}^{n}
$$

and denote by $\mathcal{B}((t, x), R)=\left[t-R^{2}, t+R^{2}\right] \times B(x, R)$ the corresponding ball of radius $R$. Remark that $(0, \infty) \times \mathbb{R}^{n}$ with this parabolic quasi-distance and the Lebesgue measure is a doubling quasi-metric measure space. The following lemma is a particular case of well-known $L^{p}\left(L^{q}\right)$ estimates for weak solutions. See [2].

Lemma 4.1. Let $q:=2+\frac{4}{n}$. There is a constant $C>0$ depending on dimension and the ellipticity constants in (1.2), such that for all u global weak solution of (1.1), for all $(t, x) \in(0, \infty) \times \mathbb{R}^{n}$, and all $r \in\left(0, \frac{\sqrt{t}}{4}\right)$, we have

$$
\left(f_{\mathcal{B}((t, x), r)}|u(s, y)|^{q} \mathrm{~d} y \mathrm{~d} s\right)^{\frac{1}{q}} \leq C\left(f_{\mathcal{B}((t, x), 4 r)}|u(s, y)|^{2} \mathrm{~d} y \mathrm{~d} s\right)^{\frac{1}{2}} .
$$

Proof. Let $(t, x) \in(0, \infty) \times \mathbb{R}^{n}$, and $r \in\left(0, \frac{\sqrt{t}}{4}\right)$. Pick $\varphi \in \mathscr{C}_{c}^{\infty}\left(\mathbb{R}^{n}\right)$ supported in $B(x, 2 r)$ such that $0 \leq \varphi \leq 1, \varphi=1$ on $B(x, r)$ and $\|\nabla \varphi\|_{\infty} \lesssim \frac{1}{r}$. Let $\sigma \in\left[t-r^{2}, t+r^{2}\right]$. By Gagliardo-Nirenberg's inequality (see [33, (2.2)]), we have that

$$
\begin{aligned}
& \int_{B(x, r)}|u(\sigma, y)|^{q} \mathrm{~d} y \leq \int|u(\sigma, y) \varphi(y)|^{q} \mathrm{~d} y \lesssim\|\nabla(u(\sigma, \cdot) \varphi)\|_{2}^{2}\|u(\sigma, \cdot) \varphi\|_{2}^{\frac{4}{n}} \\
& =\|\nabla(u(\sigma, \cdot) \varphi)\|_{2}^{2}\left(\int_{B(x, 2 r)}|u(\sigma, y)|^{2} \mathrm{~d} y\right)^{\frac{2}{n}} \\
& \lesssim\|\nabla(u(\sigma, \cdot) \varphi)\|_{2}^{2}\left(\frac{1}{r^{2}} \int_{t-r^{2}}^{t+r^{2}} \int_{B(x, 4 r)}|u(s, y)|^{2} \mathrm{~d} y \mathrm{~d} s\right)^{\frac{2}{n}}
\end{aligned}
$$

where we have used Proposition 3.6 in the last step. Now let

$$
A:=\int_{\mathcal{B}((t, x), 4 r)}|u(s, y)|^{2} \mathrm{~d} y \mathrm{~d} s .
$$

We thus have that

$$
\begin{aligned}
& \int_{\mathcal{B}((t, x), r)}|u(\sigma, y)|^{q} \mathrm{~d} y \mathrm{~d} \sigma \\
\lesssim & \left(\frac{A}{r^{2}}\right)^{\frac{2}{n}}\left[\int_{t-r^{2}}^{t+r^{2}} \int_{B(x, 2 r)}|\nabla u(\sigma, y)|^{2} \mathrm{~d} y \mathrm{~d} \sigma+\int_{t-r^{2}}^{t+r^{2}} \int_{B(x, 2 r)}|u(\sigma, y)|^{2} \frac{1}{r^{2}} \mathrm{~d} y \mathrm{~d} \sigma\right] \\
\lesssim & \left(\frac{A}{r^{2}}\right)^{\frac{2}{n}}\left[\frac{1}{r^{2}} \int_{t-16 r^{2}}^{t+16 r^{2}} \int_{B(x, 4 r)}|u(\sigma, y)|^{2} \mathrm{~d} y \mathrm{~d} \sigma\right]=\left(\frac{A}{r^{2}}\right)^{\frac{q}{2}},
\end{aligned}
$$

where we have used Proposition 3.6 again and $q=2+\frac{4}{n}$. This proves (4.1). 
Observe that the proof applies to any ball $\mathcal{B}((t, x), r)$ provided $t-16 r^{2}>0$. Hence we may apply Gerhing's lemma in the context of a space of homogeneous type. See for example a proof in [14]. As the constant $C$ is independent of $u$ and the radius of the ball, we obtain an improvement of $q$ to some $\tilde{q}>q$ that depends only on dimension and the ellipticity constants. Also, the exponent 2 can be lowered. See [25, Theorem 2] for the original euclidean proof, and [13, Theorem B1] for a proof valid in spaces of homogeneous type.

Corollary 4.2. There exist $C>0$ and $\tilde{q}>2+\frac{4}{n}$, depending on dimension and the ellipticity constants in (1.2), such that for all u global weak solution of $(1.1)$, for all $(t, x) \in(0, \infty) \times$ $\mathbb{R}^{n}$, and all $r \in\left(0, \frac{\sqrt{t}}{4}\right)$, we have, for all $p \in[1,2]$,

$$
\left(f_{\mathcal{B}((t, x), r)}|u(s, y)|^{2} \mathrm{~d} y \mathrm{~d} s\right)^{\frac{1}{2}} \lesssim\left(f_{\mathcal{B}((t, x), r)}|u(s, y)|^{\tilde{q}} \mathrm{~d} y \mathrm{~d} s\right)^{\frac{1}{\tilde{q}}} \lesssim\left(f_{\mathcal{B}((t, x), 4 r)}|u(s, y)|^{p} \mathrm{~d} y \mathrm{~d} s\right)^{\frac{1}{p}} .
$$

These reverse Hölder inequalities are useful, among other things, to control the potential growth of $L_{\text {loc }}^{2}$ norms for solutions in $L^{\infty}\left(L^{p}\right)$.

Proposition 4.3. Let $p \in[1, \infty]$. Let $u$ be a global weak solution of (1.1). Assume that $u \in L^{\infty}\left(L^{p}\right)$. Then for all $b>a>0$, and all $w \in L^{p^{\prime}}\left(\mathbb{R}^{n}\right)$,

$$
\int_{\mathbb{R}^{n}}\left(\int_{a}^{b} \int_{B(x, \sqrt{b})}|u(t, y)|^{2} \mathrm{~d} y \mathrm{~d} t\right)^{\frac{1}{2}} w(x) \mathrm{d} x<\infty .
$$

Proof. We first remark that the case $p=\infty$ is trivial, and assume from now on that $p<\infty$. By Hölder inequality, since $w \in L^{p^{\prime}}\left(\mathbb{R}^{n}\right)$, we have

$$
\begin{aligned}
I:= & \int_{\mathbb{R}^{n}}\left(\int_{a}^{b} \int_{B(x, \sqrt{b})}|u(t, y)|^{2} \mathrm{~d} y \mathrm{~d} t\right)^{\frac{1}{2}} w(x) \mathrm{d} x \\
& \lesssim\left(\int_{\mathbb{R}^{n}}\left(\int_{a}^{b} \int_{B(x, \sqrt{b})}|u(t, y)|^{2} \mathrm{~d} y \mathrm{~d} t\right)^{\frac{p}{2}} \mathrm{~d} x\right)^{\frac{1}{p}} .
\end{aligned}
$$

If $p \leq 2$, then by Corollary 4.2, and a covering argument, we have the following for all $a^{\prime} \in(0, a)$ and $b^{\prime} \in(b, \infty)$ :

$$
I \lesssim\left(\int_{a^{\prime}}^{b^{\prime}}\|u(t, \cdot)\|_{p}^{p} \mathrm{~d} t\right)^{\frac{1}{p}} \lesssim\|u\|_{L^{\infty}\left(L^{p}\right)} .
$$

If $p>2$, then by Hölder inequality

$$
\left(\int_{a}^{b} \int_{B(x, \sqrt{b})}|u(t, y)|^{2} \mathrm{~d} y \mathrm{~d} t\right)^{\frac{1}{2}} \lesssim\left(\int_{a}^{b} \int_{B(x, \sqrt{b})}|u(t, y)|^{p} \mathrm{~d} y \mathrm{~d} t\right)^{\frac{1}{p}},
$$

We conclude as in the case where $p \leq 2$ to obtain $I \lesssim\|u\|_{L^{\infty}\left(L^{p}\right)}$.

Proposition 4.4. Let $p \in[1, \infty]$ and $u$ be a global weak solution of $(1.1)$ such that $\tilde{N}(u) \in$ $L^{p}\left(\mathbb{R}^{n}\right)$. Then for all $b>a>0$, and all $w \in L^{p^{\prime}}\left(\mathbb{R}^{n}\right)$,

$$
\int_{\mathbb{R}^{n}}\left(\int_{a}^{b} \int_{B(x, \sqrt{b})}|u(t, y)|^{2} \mathrm{~d} y \mathrm{~d} t\right)^{\frac{1}{2}} w(x) \mathrm{d} x<\infty .
$$


Proof. Given $b>a>0$, there exists $M \in \mathbb{N}$ such that $b \leq 2^{M+1} a<2 b$, and there exists $N \in \mathbb{N}$ and $\left\{z_{k} ; k=1, \ldots, N\right\} \subset B(0, \sqrt{b})$ such that $B(0, \sqrt{b}) \subset \bigcup_{k=1}^{N} B\left(z_{k}, \sqrt{a}\right)$. Therefore, for all $x \in \mathbb{R}^{n}$,

$$
\begin{aligned}
\left(\int_{a}^{b} \int_{B(x, \sqrt{b})}|u(t, y)|^{2} \mathrm{~d} y \mathrm{~d} t\right)^{\frac{1}{2}} & \leq \sum_{j=0}^{M} \sum_{k=1}^{N}\left(\int_{2^{j} a}^{2^{j+1} a} \int_{B\left(x+z_{k}, \sqrt{a}\right)}|u(t, y)|^{2} \mathrm{~d} y \mathrm{~d} t\right)^{\frac{1}{2}} \\
& \lesssim \sum_{k=1}^{N} \tilde{N}(u)\left(x+z_{k}\right) .
\end{aligned}
$$

Since $w \in L^{p^{\prime}}\left(\mathbb{R}^{n}\right)$, this gives

$$
\int_{\mathbb{R}^{n}}\left(\int_{a}^{b} \int_{B(x, \sqrt{b})}|u(t, y)|^{2} \mathrm{~d} y \mathrm{~d} t\right)^{\frac{1}{2}} w(x) \mathrm{d} x \lesssim\left\|\sum_{k=1}^{N} \tilde{N}(u)\left(\cdot+z_{k}\right)\right\|\left\|_{p}\right\| w\left\|_{p^{\prime}} \lesssim\right\| \tilde{N}(u) \|_{p},
$$

using the invariance by translation of the $L^{p}$ norm.

Remark 4.5. For $p \in[1, \infty]$, note that, if $u$ is a global weak solution of $(1.1)$ such that ess sup $\|u(t, .)\|_{L^{p}}=M$, then $\sup \|u(t, .)\|_{L^{p}}=M$. This follows from the continuity of $t \mapsto$ $u(t, \cdot)$ in $L_{\text {loc }}^{2}\left(\mathbb{R}^{n}\right)$ and easy density arguments.

\subsection{Estimates for the propagators}

Lemma 4.6. Let $p \in(2, \infty]$.

(1) For all $g \in L^{2}\left(\mathbb{R}^{n}\right)$ supported in a ball $B(0, M)$, and all $t \in[0, \infty)$,

$$
\left\|\Gamma(t, 0)^{*} g\right\|_{L^{p^{\prime}}} \lesssim_{M, t}\|g\|_{L^{2}} .
$$

Consequently, for all $h \in L^{p}\left(\mathbb{R}^{n}\right), \Gamma(t, 0) h$ can be defined in $L_{\mathrm{loc}}^{2}\left(\mathbb{R}^{n}\right)$.

(2) For all $h \in L^{p}\left(\mathbb{R}^{n}\right),\|(t, x) \mapsto(\Gamma(t, 0) h)(x)\|_{X^{p}} \sim\|h\|_{L^{p}}$.

Proof. (1) Let $t \geq 0$ and $g \in L^{2}\left(\mathbb{R}^{n}\right)$ supported in a ball $B(0, M)$. Using Proposition 3.19, for some $c>0$, we have

$$
\begin{aligned}
\left\|\Gamma(t, 0)^{*} g\right\|_{L^{p^{\prime}}} & \leq \sum_{j=1}^{\infty}\left\|1_{S_{j}(0, M)} \Gamma(t, 0)^{*} g\right\|_{L^{p^{\prime}}} \lesssim M \sum_{j=1}^{\infty} 2^{j n\left(\frac{1}{p}-\frac{1}{2}\right)}\left\|1_{S_{j}(0, M)} \Gamma(t, 0)^{*} g\right\|_{L^{2}} \\
& \lesssim_{M}\|g\|_{L^{2}}+\sum_{j=2}^{\infty} 2^{j n\left(\frac{1}{p}-\frac{1}{2}\right)} e^{-c \frac{4^{j}}{t}}\|g\|_{L^{2}} \lesssim_{M, t}\|g\|_{L^{2}} .
\end{aligned}
$$

(2) Let $\delta>0, x \in \mathbb{R}^{n}$. Let $h \in L^{p}\left(\mathbb{R}^{n}\right)$, and $j \geq 1$. Using Proposition 3.19 again, we have that

$$
\begin{aligned}
\left(f_{\frac{\delta}{2}}^{\delta} f_{B(x, \sqrt{\delta})}\left|\Gamma(t, 0)\left(1_{S_{j}(x, \sqrt{\delta})} h\right)(y)\right|^{2} \mathrm{~d} y \mathrm{~d} t\right)^{\frac{1}{2}} & \lesssim 2^{\frac{j n}{2}} e^{-c 4^{j}}\left(f_{\frac{\delta}{2}}^{\delta} f_{B\left(x, 2^{j+1} \sqrt{\delta}\right)}|h(y)|^{2} \mathrm{~d} y \mathrm{~d} t\right)^{\frac{1}{2}} \\
& \lesssim 2^{\frac{j n}{2}} e^{-c 4^{j}}\left(M_{H L}|h|^{2}\right)^{\frac{1}{2}}(x)
\end{aligned}
$$


where $M_{H L}$ denote the uncentered Hardy-Littlewood maximal function. Therefore,

$$
\|\tilde{N}((t, x) \mapsto \Gamma(t, 0) h(x))\|_{L^{p}} \lesssim \sum_{j \geq 1} 2^{\frac{j n}{2}} e^{-c 4^{j}}\left\|\left(M_{H L}|h|^{2}\right)^{\frac{1}{2}}\right\|_{L^{p}} \lesssim\|h\|_{L^{p}} .
$$

We next prove the reverse inequality. Fix $z \in \mathbb{R}^{n}$. We first remark that the same reasoning as above gives us

$$
\left(f_{\frac{\delta}{2}}^{\delta} f_{B(x, \sqrt{\delta})}\left|\Gamma(t, 0)\left(1_{S_{j}(z, 1)} h\right)(y)\right|^{2} \mathrm{~d} y \mathrm{~d} t\right)^{\frac{1}{2}} \lesssim 2^{\frac{j n}{2}} e^{-c 4^{j}}\left(M_{H L}|h|^{2}\right)^{\frac{1}{2}}(x),
$$

for all $j \geq 1$ and $\delta \in(0,1)$ and $x \in B(z, 1)$. Moreover, by continuity of $t \mapsto \Gamma(t, 0)\left(1_{S_{j}(z, 1)} h\right)$ in $L^{2}\left(\mathbb{R}^{n}\right)$ and Lebesgue's differentiation theorem, we have that for all $j \geq 1$,

$$
\left(f_{\frac{\delta}{2}}^{\delta} f_{B(x, \sqrt{\delta})}\left|\Gamma(t, 0)\left(1_{S_{j}(z, 1)} h\right)(y)\right|^{2} \mathrm{~d} y \mathrm{~d} t\right)^{\frac{1}{2}} \underset{\delta \rightarrow 0}{\longrightarrow}\left|\left(1_{S_{j}(z, 1)} h\right)(x)\right|,
$$

for almost every $x \in B(z, 1)$. As the right hand side is zero for $j \geq 2$ and $x \in B(z, 1)$, we deduce by summing that

$$
\left(f_{\frac{\delta}{2}}^{\delta} f_{B(x, \sqrt{\delta})}\left|\Gamma(t, 0)\left(1_{B(z, 2)^{c}} h\right)(y)\right|^{2} \mathrm{~d} y \mathrm{~d} t\right)^{\frac{1}{2}} \underset{\delta \rightarrow 0}{\longrightarrow} 0
$$

almost everywhere for $x \in B(z, 1)$ and by difference,

$$
\left(f_{\frac{\delta}{2}}^{\delta} f_{B(x, \sqrt{\delta})}|\Gamma(t, 0) h(y)|^{2} \mathrm{~d} y \mathrm{~d} t\right)^{\frac{1}{2}} \underset{\delta \rightarrow 0}{\longrightarrow}|h(x)|,
$$

almost everywhere on $B(z, 1)$. Hence this holds on $\mathbb{R}^{n}$ as $z$ is arbitrary. Since $(t, x) \mapsto$ $(\Gamma(t, 0) h)(x) \in X^{p}$, we are done if $p=\infty$, and, if $p<\infty$, Fatou's lemma gives us

$$
\|h\|_{L^{p}} \lesssim\|(t, x) \mapsto \Gamma(t, 0) h(x)\|_{X^{p}} .
$$

Lemma 4.7. (1) Let $p \in[1, \infty)$ and $\delta>0$. We have that $\sup _{t \in[0, \delta]}\|\Gamma(t, 0)\|_{\mathcal{L}\left(E_{\delta}^{p}\right)}<\infty$.

(2) Let $p \in[1, \infty)$. For all $\delta>0$ and all $f \in E_{\delta}^{p}$, one has $\lim _{t \rightarrow 0} \Gamma(t, 0) f=f$ in $E_{\delta}^{p}$.

For all $t>0$, one also has $\lim _{s \rightarrow 0} \Gamma(t, s)^{*} f=\Gamma(t, 0)^{*} f$ in $E_{\delta}^{p}$.

(3) For all $h \in L^{\infty}\left(\mathbb{R}^{n}\right)$, we have that $\lim _{t \rightarrow 0} \Gamma(t, 0) h=h$ in $L_{\text {loc }}^{2}\left(\mathbb{R}^{n}\right)$.

Proof. (1) [12, Proposition 4.2] applies using Proposition 3.19.

(2) [12, Proposition 4.4] applies using Proposition 3.19 and the continuity results proven in Proposition 3.17 and Proposition 3.14.

(3) Let $h \in L^{\infty}\left(\mathbb{R}^{n}\right)$, and $M>0$. For $t>0$, as in Lemma 4.6 using Proposition 3.19, we see that $\sum_{j \geq 1} \mathbb{1}_{B(0, M)}(\Gamma(t, 0)-I)\left(\mathbb{1}_{S_{j}(0, M)} h\right)$ converges in $L^{2}\left(\mathbb{R}^{n}\right)$ to $\mathbb{1}_{B(0, M)}(\Gamma(t, 0)-I) h$, and moreover,

$$
\left(\int_{B(0, M)}|(\Gamma(t, 0)-I) h(y)|^{2} \mathrm{~d} y\right)^{\frac{1}{2}} \lesssim_{M}\left\|(\Gamma(t, 0)-I)\left(1_{S_{1}(0, M)} h\right)\right\|_{L^{2}}+\sum_{j=2}^{\infty} e^{-c \frac{4^{j}}{t}}\|h\|_{L^{2}},
$$

for some constant $c>0$ depending on $M$. We conclude using that $\|(\Gamma(t, 0)-I) g\|_{L^{2}} \underset{t \rightarrow 0}{\longrightarrow} 0$ for all $g \in L^{2}\left(\mathbb{R}^{n}\right)$. 
Proposition 4.8. Let $p \in(2, \infty]$. For all $h \in L^{p}\left(\mathbb{R}^{n}\right), u_{h}:(t, x) \mapsto(\Gamma(t, 0) h)(x) \in X^{p}$ is a global weak solution of (1.1).

Proof. Let $h \in L^{p}\left(\mathbb{R}^{n}\right)$. We first show that $\nabla u_{h} \in L_{\text {loc }}^{2}\left(\mathbb{R}_{+}^{n+1}\right)$. Let $a, b, M>0$. For $j \geq 1$, set $h_{j}=1_{S_{j}(0, M)} h \in L^{2}\left(\mathbb{R}^{n}\right)$ and consider the global weak solutions $u_{h_{j}}$ of (1.1) with data $h_{j}$. Applying Proposition 3.6 and Proposition 3.19, we obtain the following for some constant $\beta>0$ :

$$
\begin{aligned}
\left(\int_{a}^{b} \int_{B(0, M)}\left|\nabla\left(\Gamma(t, 0) h_{j}\right)(x)\right|^{2} \mathrm{~d} x \mathrm{~d} t\right)^{\frac{1}{2}} \\
\underset{a, b, c, M}{\lesssim}\left(\int_{a / 2}^{b} \int_{B(0,2 M)}\left|\left(\Gamma(t, 0) h_{j}\right)(x)\right|^{2} \mathrm{~d} x \mathrm{~d} t\right)^{\frac{1}{2}} \\
\lesssim e^{-\beta 4^{j}}\left\|1_{B\left(0,2^{j+1} M\right)} h\right\|_{L^{2}} \lesssim 2^{j n\left(\frac{1}{2}-\frac{1}{p}\right)} e^{-\beta 4^{j}}\|h\|_{L^{p}}
\end{aligned}
$$

We easily obtain from this that $\sum_{j \geq 1} \nabla u_{h_{j}}$ converges to $\nabla u_{h} \in L^{2}(a, b ; B(0, M))$. Also $\sum_{j \geq 1} u_{h_{j}}$ converges to $u_{h}$ in $L^{2}(a / 2, b ; B(0,2 M))$

To show that $u_{h}$ satisfies (1.1) in the sense of distributions, let $\phi \in \mathscr{D}$ and pick $a, b, M>0$ such that $\operatorname{supp} \phi \subset[a, b] \times B(0, M)$. For each $j \geq 1$,

$$
-\int_{0}^{\infty} \int_{\mathbb{R}^{n}} u_{h_{j}} \overline{\partial_{t} \phi} \mathrm{d} x \mathrm{~d} t+\int_{0}^{\infty} \int_{\mathbb{R}^{n}} A \nabla u_{h_{j}} \cdot \overline{\nabla \phi} \mathrm{d} x \mathrm{~d} t=0
$$

and by the above $L^{2}$ convergences, one can sum in $j \geq 1$ and obtain the conclusion for $u_{h}$. This shows it is a global weak solution of (1.1).

Lemma 4.9. Let $q \in[1,2)$ and assume that $\sup _{0 \leq s \leq t<\infty}\|\Gamma(t, s)\|_{\mathscr{L}\left(L^{q}\right)}<\infty$. Then, for all $r \in(q, 2]$ there exists $\alpha>0$ such that for all $E, F \subset \mathbb{R}^{n}$ Borel sets, for all $0 \leq s<t<\infty$ and all $f \in L^{r}\left(\mathbb{R}^{n}\right)$,

$$
\left\|1_{E} \Gamma(t, s)\left(\mathbb{1}_{F} f\right)\right\|_{L^{2}} \lesssim(t-s)^{-\frac{n}{2}\left(\frac{1}{r}-\frac{1}{2}\right)} e^{-\alpha \frac{d(E, F)^{2}}{t-s}}\left\|1_{F} f\right\|_{L^{r}}
$$

Proof. Let $f \in L^{2}\left(\mathbb{R}^{n}\right) \cap L^{q}\left(\mathbb{R}^{n}\right), x \in \mathbb{R}^{n}, t>s \geq 0$. By Proposition 3.6 we have that

$$
\left(f_{B(x, \sqrt{t-s})}|\Gamma(t, s) f(y)|^{2} \mathrm{~d} y\right)^{\frac{1}{2}} \lesssim\left(f_{s}^{t} f_{B(x, 2 \sqrt{t-s})}|\Gamma(\sigma, s) f(y)|^{2} \mathrm{~d} y \mathrm{~d} \sigma\right)^{\frac{1}{2}} .
$$

Covering $B(x, 2 \sqrt{t-s})$ by a finite collection of balls $\left\{B\left(x_{j}, \frac{\sqrt{t-s}}{2}\right) ; j=1, \ldots, M\right\}$ with $M$ depending only on $n$ and $B\left(x_{j}, \frac{\sqrt{t-s}}{2}\right) \subset B(x, 4 \sqrt{t-s})$ for all $j=1, \ldots, M$, we can apply Corollary 4.2 to obtain

$$
\begin{aligned}
\left(f_{B(x, \sqrt{t-s})}|\Gamma(t, s) f(y)|^{2} \mathrm{~d} y\right)^{\frac{1}{2}} & \lesssim\left(f_{s}^{t} f_{B(x, 4 \sqrt{t-s})}|\Gamma(\sigma, s) f(y)|^{q} \mathrm{~d} y \mathrm{~d} \sigma\right)^{\frac{1}{q}} \\
& \lesssim(t-s)^{-\frac{n}{2 q}}\left(f_{s}^{t}\|f\|_{q}^{q} \mathrm{~d} \sigma\right)^{\frac{1}{q}}=(t-s)^{-\frac{n}{2 q}}\|f\|_{q} .
\end{aligned}
$$

Therefore, for all $x \in \mathbb{R}^{n}, t>s \geq 0$ and $h \in L^{q}\left(\mathbb{R}^{n}\right)$

$$
\left\|1_{B(x, \sqrt{t-s})} \Gamma(t, s) h\right\|_{L^{2}} \lesssim(t-s)^{-\frac{n}{2}\left(\frac{1}{q}-\frac{1}{2}\right)}\|h\|_{L^{q}} .
$$


Let $\delta=\sqrt{t-s}$. Consider the family of disjoint cubes $\mathscr{D}_{\delta}:=\left\{\delta\left[0,1\left[{ }^{n}+k \delta, k \in \mathbb{Z}^{n}\right\}\right.\right.$. We denote by $c_{Q}$ the center of a cube $Q \in \mathscr{D}_{\delta}$. We have that

$$
(t-s)^{\frac{n}{2}\left(\frac{1}{q}-\frac{1}{2}\right)} 1_{Q} \Gamma(t, s) \mathbb{1}_{R} \in \mathscr{L}\left(L^{q}\left(\mathbb{R}^{n}\right), L^{2}\left(\mathbb{R}^{n}\right)\right)
$$

with norm independent of $t>s \geq 0$ and $Q, R \in \mathscr{D}_{\delta}$. Using Proposition 3.19 with RieszThorin interpolation, we have that, for all $r \in(q, 2]$, there exists $\alpha_{r}>0$ such that for all $h \in L^{r}\left(\mathbb{R}^{n}\right)$

$$
\left\|1_{Q} \Gamma(t, s)\left(\mathbb{1}_{R} h\right)\right\|_{L^{2}} \lesssim(t-s)^{-\frac{n}{2}\left(\frac{1}{r}-\frac{1}{2}\right)} e^{-\alpha_{r} \frac{d(Q, R)^{2}}{t-s}}\left\|1_{R} h\right\|_{L^{r}},
$$

for all $Q, R \in \mathscr{D}_{\delta}$, for all $t>s \geq 0$. Therefore, there exists $c_{r}^{\prime}>0$ such that

$$
\begin{aligned}
\|\Gamma(t, s) h\|_{L^{2}} & =\left(\sum_{Q \in \mathscr{D}_{\delta}}\left\|1_{Q} \Gamma(t, s) h\right\|_{L^{2}}^{2}\right)^{\frac{1}{2}} \\
& \lesssim \sum_{k \in \mathbb{Z}^{n}}\left(\sum_{Q \in \mathscr{D}_{\delta}}\left\|1_{Q+k \delta} \Gamma(t, s)\left(1_{Q} h\right)\right\|_{L^{2}}^{2}\right)^{\frac{1}{2}} \\
& \lesssim \sum_{k \in \mathbb{Z}^{n}} e^{-c_{r}^{\prime}|k|}(t-s)^{-\frac{n}{2}\left(\frac{1}{r}-\frac{1}{2}\right)}\left(\sum_{Q \in \mathscr{D}_{\delta}}\left\|1_{Q} h\right\|_{L^{r}}^{2}\right)^{\frac{1}{2}} \\
& \lesssim(t-s)^{-\frac{n}{2}\left(\frac{1}{r}-\frac{1}{2}\right)}\left(\sum_{Q \in \mathscr{D}_{\delta}}\left\|1_{Q} h\right\|_{L^{r}}^{r}\right)^{\frac{1}{r}}=(t-s)^{-\frac{n}{2}\left(\frac{1}{r}-\frac{1}{2}\right)}\|h\|_{L^{r}},
\end{aligned}
$$

where we have used that $\ell_{r} \subset \ell_{2}$ since $r \leq 2$. Therefore

$$
\|\Gamma(t, s)\|_{\mathscr{L}\left(L^{r}, L^{2}\right)} \lesssim(t-s)^{-\frac{n}{2}\left(\frac{1}{r}-\frac{1}{2}\right)}
$$

uniformly for $0 \leq s<t<\infty$. Using Riesz-Thorin interpolation again to interpolate this uniform bound with the $L^{2}-L^{2}$ off diagonal bound from Proposition 3.19 gives the result.

Lemma 4.10. Let $q \in[1,2)$, and assume that $\sup _{0 \leq s \leq t<\infty}\|\Gamma(t, s)\|_{\mathcal{L}\left(L^{q}\right)}<\infty$. Then, for all $h \in L^{q}\left(\mathbb{R}^{n}\right), u_{h}:(t, x) \mapsto(\Gamma(t, 0) h)(x)$ is a global weak solution of (1.1).

Proof. By (4.4), we have that, for all $t>0$, all $h \in L^{q}\left(\mathbb{R}^{n}\right)$, and all $M>0$ :

$$
\left\|1_{B(0, M)} \Gamma(t, 0) h\right\|_{L^{2}} \lesssim_{M, t}\|h\|_{L^{q}} .
$$

Applying Proposition 3.6, we obtain the following for all $c \in(a, b)$ and $M>0$ :

$$
\left(\int_{c}^{b} \int_{B(0, M)}|\nabla \Gamma(t, 0) h(x)|^{2} \mathrm{~d} x \mathrm{~d} t\right)^{\frac{1}{2}} \underset{a, b, c, M}{\lesssim}\left(\int_{a}^{b}\left\|1_{B(0,2 M)} \Gamma(t, 0) h\right\|_{L^{2}}^{2} d t\right)^{\frac{1}{2}} \lesssim\|h\|_{L^{q}} .
$$

To show that $u_{h}$ satisfies (1.1) in the sense of distributions, let $\varepsilon>0$, and pick $h_{0} \in$ $L^{q}\left(\mathbb{R}^{n}\right) \cap L^{2}\left(\mathbb{R}^{n}\right)$ such that $\left\|h-h_{0}\right\|_{L^{q}}<\varepsilon$. The function $u_{h_{0}}$ is a global weak solution of (1.1), and we thus have the following.

$$
\begin{aligned}
\mid & -\int_{0}^{\infty} \int_{\mathbb{R}^{n}} u_{h} \overline{\partial_{t} \phi}+\int_{0}^{\infty} \int_{\mathbb{R}^{n}} A \nabla u_{h} \cdot \overline{\nabla \phi} \mid \\
& \leq \int_{0}^{\infty} \int_{\mathbb{R}^{n}}\left|u_{h-h_{0}}\right|\left|\partial_{t} \phi\right|+\Lambda \int_{0}^{\infty} \int_{\mathbb{R}^{n}}\left|\nabla u_{h-h_{0}}\right||\nabla \phi| \\
& \lesssim\left\|u_{h-h_{0}}\right\|_{L^{\infty}\left(L^{q}\right)}+\left\|\nabla\left(u_{h-h_{0}}\right)\right\|_{L^{2}(\operatorname{supp}(\nabla \phi))} \lesssim\left\|h-h_{0}\right\|_{L^{q}}<\varepsilon .
\end{aligned}
$$

This proves that $u_{h}$ is a global weak solution of (1.1). 
Lemma 4.11. Let $q \in(2, \infty]$ and assume that $\sup _{0 \leq s \leq t<\infty}\|\Gamma(t, s)\|_{\mathscr{L}\left(L^{q}\right)}<\infty$. Then, for all $r \in[2, q)$ there exists $\alpha>0$ such that for all $E, F \subset \mathbb{R}^{n}$ Borel sets, for all $0 \leq s<t<\infty$ and all $f \in L^{2}\left(\mathbb{R}^{n}\right)$,

$$
\left\|1_{E} \Gamma(t, s)\left(\mathbb{1}_{F} f\right)\right\|_{L^{r}} \lesssim(t-s)^{-\frac{n}{2}\left(\frac{1}{2}-\frac{1}{r}\right)} e^{-\alpha \frac{d(E, F)^{2}}{t-s}}\left\|11_{F} f\right\|_{L^{2}} .
$$

Proof. Using Proposition 3.19 we only have to show that

$$
\sup _{0 \leq s<t<\infty}\left\|(t-s)^{-\frac{n}{2}\left(\frac{1}{q^{\prime}}-\frac{1}{2}\right)} \Gamma(t, s)^{*}\right\|_{\mathscr{L}\left(L^{q^{\prime}}, L^{2}\right)}<\infty .
$$

For $0 \leq s \leq t<\infty$, we have by Proposition 3.17 and the proof of Lemma 3.16:

$$
\Gamma(t, s)^{*}=\tilde{\Gamma}(s, t)=\underset{\sim}{\Gamma}(t-s, 0),
$$

where $\underset{\sim}{\Gamma}$ is the propagator for equation (1.1) with $A$ replaced by

$$
\tilde{A}(s, x)=\left\{\begin{array}{l}
A^{*}(t-s, x) \text { if }(s, x) \in[0, t] \times \mathbb{R}^{n}, \\
A^{*}(0, x) \text { otherwise. }
\end{array}\right.
$$

Since $\tilde{A}$ satisfies (1.2) with the same constants as $A$, Proposition 3.19 applies to $\tilde{\Gamma}$ and the result follows from interpolation between $q^{\prime}$ and 2 .

Remark 4.12. For $p \in[1, \infty]$,

$$
\operatorname{esssup}_{0 \leq s \leq t<\infty}\|\Gamma(t, s)\|_{\mathcal{L}\left(L^{p}\right)}=M \quad \Longrightarrow \quad \sup _{0 \leq s \leq t<\infty}\|\Gamma(t, s)\|_{\mathcal{L}\left(L^{p}\right)}=M .
$$

This follows, using Proposition 3.14 and Proposition 3.17, from the continuity of $t \mapsto$ $\langle\Gamma(t, s) f, g\rangle$ on $\left[s, \infty\right.$ ) and of $s \mapsto\langle\Gamma(t, s) f, g\rangle$ on $[0, t]$ for all $f, g \in \mathscr{D}$ (or $f \in L^{\infty}, g \in L^{1}$ compactly supported), a simple measure theoretical argument and density arguments.

\subsection{Propagators with kernel bounds}

We say that the propagators $\Gamma(t, s), 0 \leq s<t<\infty$, have kernel bounds if their kernels $k(t, s, . .$.$) are measurable functions with$

$$
|k(t, s, x, y)| \leq C(t-s)^{-\frac{n}{2}} e^{-c \frac{|x-y|^{2}}{4(t-s)}},
$$

for some $C, c>0$, all $0 \leq s<t<\infty$, and almost all $x, y \in \mathbb{R}^{n}$.

In this case, $\Gamma(t, s)$ is an integral operator and one has the integral representation

$$
\Gamma(t, s) f(x)=\int_{\mathbb{R}^{n}} k(t, s, x, y) f(y) \mathrm{d} y
$$

for all $f \in L^{2}\left(\mathbb{R}^{n}\right)$ and almost every $x \in \mathbb{R}^{n}$. Moreover, as the integral makes sense for $f \in L^{p}\left(\mathbb{R}^{n}\right), 1 \leq p \leq \infty$, one can extend $\Gamma(t, s)$ to a bounded operator on $L^{p}\left(\mathbb{R}^{n}\right)$, uniformly in $t \geq s$ (recall that $\Gamma(s, s)=I$ ).

As mentioned, Aronson's proved kernel bounds for propagators of real equations. At this point, it is worth pointing out that the following result, proven by Hofmann and Kim in [21, Theorems 1.1 and 1.2], extends to our situation. 
Proposition 4.13. (1) The propagators $\Gamma(t, s), 0 \leq s \leq t<\infty$, have kernel bounds if weak solutions in $L_{\mathrm{loc}}^{2}\left(\overline{\mathbb{R}_{+}^{n+1}}\right)$ of (1.1) and of (3.2) on $\mathbb{R}_{+}^{n+1}$ satisfy scale invariant local $L^{2}-L^{\infty}$ bounds of Moser type on parabolic cylinders. The constants $C, c$ in (4.5) depend on the ellipticity constants in (1.2) and the bounds in the local estimates.

(2) Conversely, if the propagators $\Gamma(t, s), 0 \leq s \leq t<\infty$, have kernel bounds then global weak solutions satisfy the scale invariant local $L^{2}-L^{\infty}$ bounds of Moser type on Whitney parabolic cylinders.

The proof in [21] is done for smooth coefficients. In this case, one can use the classical fundamental solution. However, once we have our notion of propagators, we can run the argument mutatis mutandi. In particular, supremum is replaced by essential supremum (or even supremum in time and essentiel supremum in $x$ ) in the local bounds. Also the argument for the first part is done for the propagators on the full range $-\infty<s<t<\infty$, but inspection reveals that, to get the estimate for $k(s, t, x, y)$, only local bounds on parabolic cylinders contained in the strip $[s, t] \times \mathbb{R}^{n}$ are used. This explains our hypothesis on the weak solutions in part (1).

The converse is stated in [21, Theorems 1.2] for the full range. The argument there does not preserve strips $[s, t] \times \mathbb{R}^{n}$ (a modification of the argument could probably do it) but, if we restrict to parabolic cylinders of Whitney type (as in the definition of the maximal function $\tilde{N}$ ), then the argument gives the desired local bounds.

\section{$5 \quad$ Existence and uniqueness results}

\subsection{Main result}

Here, we prove interior representation from a weak control on solutions.

Theorem 5.1. Let $u$ be a local weak solution of $(1.1)$ on $(a, b) \times \mathbb{R}^{n}$. Assume

$$
M:=\int_{\mathbb{R}^{n}}\left(\int_{a}^{b} \int_{B(x, \sqrt{b})}|u(t, y)|^{2} \mathrm{~d} y \mathrm{~d} t\right)^{\frac{1}{2}} e^{-\gamma|x|^{2}} \mathrm{~d} x<\infty
$$

for some $\gamma<\frac{\alpha}{4(b-a)}$ where $\alpha$ is the constant in Proposition $3.19\left(\alpha=\frac{\lambda}{4 \Lambda^{2}}\right)$. Then $u(t, \cdot)=$ $\Gamma(t, s) u(s, \cdot)$ for every $a<s \leq t<b$, in the following sense:

$$
\int_{\mathbb{R}^{n}} u(s, x) \overline{\Gamma(t, s)^{*} h(x)} \mathrm{d} x=\int_{\mathbb{R}^{n}} u(t, x) \overline{h(x)} \mathrm{d} x \quad \forall h \in \mathscr{C}_{c}\left(\mathbb{R}^{n}\right) .
$$

Proof. Step 0: For $h \in \mathscr{C}_{c}\left(\mathbb{R}^{n}\right)$, its support being included in $B(0, \rho)$ for some $\rho>0$, we have for all $j \geq 1$ and for all $x \in \mathbb{R}^{n}$ that

$$
e^{-\beta 4^{j}}\left\|1_{S_{j}(x, \sqrt{b})} h\right\|_{L^{2}} \lesssim\left(\mathbb{1}_{|x| \leq \rho}+e^{-\beta \frac{(|x|-\rho)^{2}}{4 b}} \mathbb{1}_{|x|>\rho}\right)\|h\|_{L^{2}}
$$

(recall that $S_{j}(x, \sqrt{b})$ denotes the annulus $B\left(x, 2^{j+1} \sqrt{b}\right) \backslash B\left(x, 2^{j} \sqrt{b}\right)$ if $j \geq 2$ and the ball $B(x, 2 \sqrt{b})$ if $j=1)$ and therefore, for $\gamma<\frac{\beta}{4 b}$,

$$
\sup _{x \in \mathbb{R}^{n}}\left(e^{\gamma|x|^{2}} e^{-\beta 4^{j}}\left\|1_{S_{j}(x, \sqrt{b})} h\right\|_{L^{2}}\right) \lesssim\|h\|_{L^{2}}, \quad \forall j \in \mathbb{N} .
$$


If $|x| \leq \rho$, then (5.1) is immediate. Let $|x|>\rho:\left\|1_{S_{j}(x, \sqrt{b})} h\right\|_{2} \neq 0$ only if $B(0, \rho) \cap$ $S_{j}(x, \sqrt{b}) \neq \emptyset$. Pick $y \in B(0, \rho) \cap S_{j}(x, \sqrt{b})$ and we have that

$$
|x| \leq|x-y|+|y| \leq 2^{j+1} \sqrt{b}+\rho, \quad \text { and then } \frac{1}{4 b}(|x|-\rho)^{2} \leq 4^{j} .
$$

This implies (5.1). Now, for $\gamma<\frac{\beta}{4 b}$, we have that

$$
\sup _{x \in \mathbb{R}^{n}}\left(e^{\gamma|x|^{2}} e^{-\beta \frac{(|x|-\rho)^{2}}{4 b}}\right)=e^{\frac{\beta \gamma \rho^{2}}{\beta-4 b \gamma}}<\infty \quad \text { and } \quad \sup _{x \in \mathbb{R}^{n}}\left(\mathbb{1}_{|x| \leq \rho} e^{\gamma|x|^{2}}\right)=e^{\gamma \rho^{2}}<\infty,
$$

which proves (5.2).

Step 1: We show that for all $a<s \leq t<b$ and all $h \in \mathscr{C}_{c}\left(\mathbb{R}^{n}\right)$, we have that $u(s, \cdot) \overline{\Gamma(t, s)^{*} h} \in$ $L^{1}\left(\mathbb{R}^{n}\right)$.

Let $\rho>0$ be such that $\operatorname{supp} h \subset B(0, \rho)$. Using Proposition 3.6 and Proposition 3.19, we have the following (with constants depending on $t, s, b, \rho$ ):

$$
\begin{array}{rl}
\int_{\mathbb{R}^{n}} & u(s, x) \| \Gamma(t, s)^{*} h(x) \mid \mathrm{d} x=\int_{\mathbb{R}^{n}}\left(f_{B\left(x, \frac{\sqrt{b}}{2}\right)}\left|u(s, y) \| \Gamma(t, s)^{*} h(y)\right| \mathrm{d} y\right) \mathrm{d} x \\
& \leq \sum_{j=1}^{\infty} \int_{\mathbb{R}^{n}}\left(f_{B\left(x, \frac{\sqrt{b}}{2}\right)}|u(s, y)|^{2} \mathrm{~d} y\right)^{\frac{1}{2}}\left\|1_{B\left(x, \frac{\sqrt{b}}{2}\right)} \Gamma(t, s)^{*}\left(1_{S_{j}(x, \sqrt{b})} h\right)\right\|_{L^{2}} \mathrm{~d} x \\
& \lesssim \sum_{j=1}^{\infty} \int_{\mathbb{R}^{n}}\left(\int_{a}^{b} \int_{B(x, \sqrt{b})}|u(\sigma, y)|^{2} \mathrm{~d} y \mathrm{~d} \sigma\right)^{\frac{1}{2}} e^{-\alpha \frac{4^{j} b}{b-a}}\left\|1_{S_{j}(x, \sqrt{b})} h\right\|_{L^{2}} \mathrm{~d} x \\
& \lesssim M\|h\|_{L^{2}}<\infty .
\end{array}
$$

where we have used, for any $\varepsilon>0, \alpha \frac{4^{j} b}{b-a}=\varepsilon 4^{j}+\beta 4^{j}$, the fact that $\sum_{j=1}^{\infty} e^{-\varepsilon 4^{j}}<\infty$ and (5.2) with $\beta=\frac{\alpha b}{(b-a)}-\varepsilon$ and $\gamma<\frac{\beta}{4 b}$ in the last line.

Step 2: Some identities.

We fix $h \in \mathscr{C}_{c}\left(\mathbb{R}^{n}\right)$ and let $a<t<b$. Define $\phi(s, x):=\Gamma(t, s)^{*} h(x)$ for all $s \in[0, t]$ and $x \in \mathbb{R}^{n}$. By construction, the function $\phi$ is a weak solution of the backward equation (3.2) with $\nabla \phi \in L^{2}\left(0, t ; L^{2}\left(\mathbb{R}^{n}\right)\right)$ and one has $\phi \in \mathscr{C}\left([0, t] ; L^{2}\left(\mathbb{R}^{n}\right)\right)$. Let $\chi \in \mathscr{C}_{c}^{\infty}\left(\mathbb{R}^{n} ; \mathbb{R}\right)$, and let $\eta \in \mathscr{C}_{c}^{\infty}((a, t) ; \mathbb{R})$. Denote by $\Omega$ a bounded open set containing the support of $\chi$. Since $u$ is a weak solution of (1.1), we have that

$L^{2}\left(a, b ; H^{-1}(\Omega)\right)\left\langle\partial_{s} u, \phi \chi^{2} \eta\right\rangle_{L^{2}\left(a, b ; H_{0}^{1}(\Omega)\right)}=-\int_{a}^{b} \int_{\Omega} A(s, x) \nabla u(s, x) \cdot \nabla\left(\overline{\phi(s, x)} \chi^{2}(x) \eta(s)\right) \mathrm{d} x \mathrm{~d} s$.

Since $\phi$ is a weak solution of (3.2), $\nabla u \in L_{\text {loc }}^{2}$ and $u \in \mathscr{C}\left([a, b], L_{\text {loc }}^{2}\right)$ by Proposition 3.6, we have that

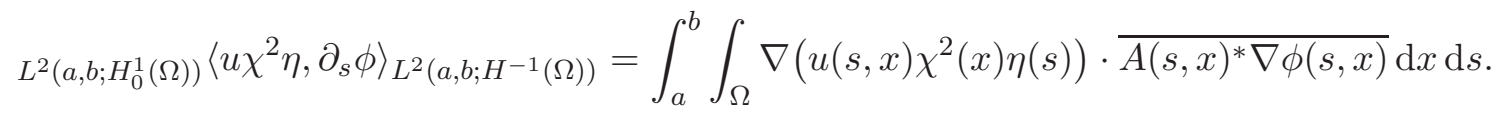

Noting that

$$
\begin{gathered}
L^{2\left(H^{-1}\right)}\left\langle\partial_{s} u, \phi \chi^{2} \eta\right\rangle_{L^{2}\left(H_{0}^{1}\right)}+{ }_{L^{2}\left(H_{0}^{1}\right)}\left\langle u \chi^{2} \eta, \partial_{s} \phi\right\rangle_{L^{2}\left(H^{-1}\right)}+\int_{a}^{b} \int_{\Omega} \chi^{2}(x) u(s, x) \overline{\phi(s, x)} \eta^{\prime}(s) \mathrm{d} x \mathrm{~d} s \\
=\int_{a}^{b} \partial_{s}\left(\int_{\Omega}\left(u(s, x) \overline{\phi(s, x)} \chi^{2}(x) \eta(s)\right) \mathrm{d} x\right) \mathrm{d} s=0
\end{gathered}
$$


we get, adding the three equations above,

$$
\begin{aligned}
& \int_{a}^{b} \int_{\Omega} \chi^{2}(x) u(s, x) \overline{\phi(s, x)} \eta^{\prime}(s) \mathrm{d} x \mathrm{~d} s \\
= & \int_{a}^{b} \eta(s) \int_{\Omega}\left(A(s, x) \nabla u(s, x) \cdot \nabla\left(\overline{\phi(s, x)} \chi^{2}(x)\right)-\nabla\left(u(s, x) \chi^{2}(x)\right) \cdot \overline{A(s, x)^{*} \nabla \phi(s, x)}\right) \mathrm{d} x \mathrm{~d} s .
\end{aligned}
$$

Calculating, some terms cancel and we obtain

$$
\begin{aligned}
& \int_{a}^{b} \int_{\Omega} \chi^{2}(x) u(s, x) \overline{\phi(s, x)} \eta^{\prime}(s) \mathrm{d} x \mathrm{~d} s \\
= & \int_{a}^{b} \eta(s) \int_{\Omega}\left(\left(A(s, x) \nabla u(s, x) \cdot \nabla \chi^{2}(x)\right) \overline{\phi(s, x)}-u(s, x)\left(\nabla \chi^{2}(x) \cdot \overline{A(s, x)^{*} \nabla \phi(s, x)}\right)\right) \mathrm{d} x \mathrm{~d} s .
\end{aligned}
$$

Step 3: We now prove that

$$
\int_{a}^{b} \int_{\mathbb{R}^{n}} u(s, x) \overline{\phi(s, x)} \eta^{\prime}(s) \mathrm{d} x \mathrm{~d} s=0 .
$$

We choose $\chi$ of the form $x \mapsto \psi\left(\frac{|x|}{R}\right)$ for $R>0$ and $\psi \in \mathscr{C}_{c}^{\infty}([0, \infty))$ supported on $[0,2]$, and equal to 1 on $[0,1]$. Note that $\chi(y)=1$ for all $y \in B(0, R)$ and $\|\nabla \chi\|_{\infty} \lesssim R^{-1}$. We have already shown that $u(s, \cdot) \overline{\phi(s, \cdot)} \in L^{1}\left(\mathbb{R}^{n}\right)$ for every $s \in(a, t]$. Thus the left hand side of (5.3) goes to the left hand side of (5.4) as $R$ goes to $\infty$ by dominated convergence. To prove (5.4), it remains to show that $\bar{\phi} \nabla u \in L^{1}\left((c, d) \times \mathbb{R}^{n}\right)$ and that $u \overline{\nabla \phi} \in L^{1}\left((c, d) \times \mathbb{R}^{n}\right)$ with $a<c<d<t$ such that $\operatorname{supp} \eta \subset[c, d]$, so that dominated convergence applies as well as $R$ goes to $\infty$. Using Proposition 3.6 and Proposition 3.19, this is done as a simple modification of the argument used in Step 0 and Step 1.

$$
\begin{aligned}
& \int_{c}^{d} \int_{\mathbb{R}^{n}}|\nabla u(s, x)|\left|\Gamma(t, s)^{*} h(x)\right| \mathrm{d} x \mathrm{~d} s=\int_{c}^{d} \int_{\mathbb{R}^{n}}\left(f_{B\left(x, \frac{\sqrt{b}}{2}\right)}\left|\nabla u(s, y) \| \Gamma(t, s)^{*} h(y)\right| \mathrm{d} y\right) \mathrm{d} x \mathrm{~d} s \\
& \lesssim \sum_{j=1}^{\infty} \int_{\mathbb{R}^{n}}\left(\int_{c}^{b} f_{B\left(x, \frac{\sqrt{b}}{2}\right)}|\nabla u(s, y)|^{2} \mathrm{~d} y \mathrm{~d} s\right)^{\frac{1}{2}}\left(\int_{c}^{d}\left\|1_{B\left(x, \frac{\sqrt{b}}{2}\right)} \Gamma(t, s)^{*}\left(1_{S_{j}(x, \sqrt{b})} h\right)\right\|_{L^{2}}^{2} \mathrm{~d} s\right)^{\frac{1}{2}} \mathrm{~d} x \\
& \lesssim \sum_{j=1}^{\infty} \int_{\mathbb{R}^{n}}\left(\int_{a}^{b} \int_{B(x, \sqrt{b})}|u(s, y)|^{2} \mathrm{~d} y \mathrm{~d} s\right)^{\frac{1}{2}} e^{-\frac{\alpha b}{b-a} 4^{j}}\left\|1_{S_{j}(x, \sqrt{b})} h\right\|_{L^{2}} \mathrm{~d} x .
\end{aligned}
$$

We conclude by (5.2) with $\beta<\frac{\alpha b}{(b-a)}$ and this gives $\bar{\phi} \nabla u \in L^{1}\left((c, d) \times \mathbb{R}^{n}\right)$. Using Proposition 3.9, instead of Proposition 3.6, and Proposition 3.19, and $d<t$ :

$$
\begin{aligned}
& \int_{c}^{d} \int_{\mathbb{R}^{n}}|u(s, x)|\left|\nabla \Gamma(t, s)^{*} h(x)\right| \mathrm{d} x \mathrm{~d} s=\int_{c}^{d} \int_{\mathbb{R}^{n}}\left(f_{B\left(x, \frac{\sqrt{b}}{2}\right)}\left|u(s, y) \| \nabla \Gamma(t, s)^{*} h(y)\right| \mathrm{d} y\right) \mathrm{d} x \mathrm{~d} s \\
& \lesssim \sum_{j=1}^{\infty} \int_{\mathbb{R}^{n}}\left(\int_{a}^{b} f_{B\left(x, \frac{\sqrt{b}}{2}\right)}|u(s, y)|^{2} \mathrm{~d} y \mathrm{~d} s\right)^{\frac{1}{2}}\left(\int_{c}^{d}\left\|1_{B\left(x, \frac{\sqrt{b}}{2}\right)} \nabla \Gamma(t, s)^{*}\left(1_{S_{j}(x, \sqrt{b})} h\right)\right\|_{L^{2}}^{2} \mathrm{~d} s\right)^{\frac{1}{2}} \mathrm{~d} x \\
& \lesssim \sum_{j=1}^{\infty} \int_{\mathbb{R}^{n}}\left(\int_{a}^{b} \int_{B(x, \sqrt{b})}|u(s, y)|^{2} \mathrm{~d} y \mathrm{~d} s\right)^{\frac{1}{2}}\left(\int_{a}^{t}\left\|1_{B(x, \sqrt{b})} \Gamma(t, s)^{*}\left(1_{S_{j}(x, \sqrt{b})} h\right)\right\|_{L^{2}}^{2} \mathrm{~d} s\right)^{\frac{1}{2}} \mathrm{~d} x \\
& \lesssim \sum_{j=1}^{\infty} \int_{\mathbb{R}^{n}}\left(\int_{a}^{b} \int_{B(x, \sqrt{b})}|u(s, y)|^{2} \mathrm{~d} y \mathrm{~d} s\right)^{\frac{1}{2}} e^{-\alpha \frac{4^{j} b}{b-a}}\left\|1_{S_{j}(x, \sqrt{b})} h\right\|_{L^{2}} \mathrm{~d} x .
\end{aligned}
$$


By (5.2) with $\beta<\frac{\alpha b}{(b-a)}$, this gives $u \overline{\nabla \phi} \in L^{1}\left((c, d) \times \mathbb{R}^{n}\right)$. We have thus established (5.4). Step 4: Choosing a specific $\eta$.

We now pick $v \in \mathscr{C}_{c}^{\infty}\left(-\frac{1}{2}, \frac{1}{2}\right)$ such that $\int_{-\frac{1}{2}}^{\frac{1}{2}} v(y) d y=1$. For $\varepsilon \in\left(0, \frac{t-s}{3} \wedge \frac{s-a}{3}\right)$, we consider $\eta \in \mathscr{C}_{c}^{\infty}(a, t)$ such that

$$
\eta^{\prime}(\sigma)=\frac{1}{2 \varepsilon} v\left(\frac{\sigma-(s-\varepsilon)}{2 \varepsilon}\right)-\frac{1}{2 \varepsilon} v\left(\frac{\sigma-(t-2 \varepsilon)}{2 \varepsilon}\right) \quad \forall \sigma \in(a, t) .
$$

Remark that the support of $\eta^{\prime}$ is contained in $[a+\varepsilon, t-\varepsilon]$ and as it has mean value 0 , the same hold for $\eta$. From (5.4) (with $s$ becoming $\sigma$ in the integral), we thus get that

$$
\begin{aligned}
& \frac{1}{2 \varepsilon} \int_{s-2 \varepsilon}^{s} v\left(\frac{\sigma-(s-\varepsilon)}{2 \varepsilon}\right)\left(\int_{\mathbb{R}^{n}} u(\sigma, x) \bar{\phi}(\sigma, x) \mathrm{d} x\right) \mathrm{d} \sigma \\
= & \frac{1}{2 \varepsilon} \int_{t-3 \varepsilon}^{t-\varepsilon} v\left(\frac{\sigma-(t-2 \varepsilon)}{2 \varepsilon}\right)\left(\int_{\mathbb{R}^{n}} u(\sigma, x) \bar{\phi}(\sigma, x) \mathrm{d} x\right) \mathrm{d} \sigma,
\end{aligned}
$$

and thus, changing variables:

$$
\begin{aligned}
& \int_{-\frac{1}{2}}^{\frac{1}{2}} v(\sigma)\left(\int_{\mathbb{R}^{n}} u(s-\varepsilon(1-2 \sigma), x) \bar{\phi}(s-\varepsilon(1-2 \sigma), x) \mathrm{d} x\right) \mathrm{d} \sigma \\
= & \int_{-\frac{1}{2}}^{\frac{1}{2}} v(\sigma)\left(\int_{\mathbb{R}^{n}} u(t-2 \varepsilon(1-\sigma), x) \bar{\phi}(t-2 \varepsilon(1-\sigma), x) \mathrm{d} x\right) \mathrm{d} \sigma .
\end{aligned}
$$

Recall that $\phi(t, x)=h(x)$ and $\phi(s, x)=\Gamma(t, s)^{*} h(x)$. The result will be proven once we have established that

$$
\lim _{\varepsilon \rightarrow 0} \int_{-\frac{1}{2}}^{\frac{1}{2}} v(\sigma) \int_{\mathbb{R}^{n}} u(t-2 \varepsilon(1-\sigma), x) \bar{\phi}(t-2 \varepsilon(1-\sigma), x) \mathrm{d} x \mathrm{~d} \sigma=\int_{\mathbb{R}^{n}} u(t, x) \bar{\phi}(t, x) \mathrm{d} x
$$

and that

$$
\lim _{\varepsilon \rightarrow 0} \int_{-\frac{1}{2}}^{\frac{1}{2}} v(\sigma) \int_{\mathbb{R}^{n}} u(s-\varepsilon(1-2 \sigma), x) \bar{\phi}(s-\varepsilon(1-2 \sigma), x) \mathrm{d} x \mathrm{~d} \sigma=\int_{\mathbb{R}^{n}} u(s, x) \bar{\phi}(s, x) \mathrm{d} x,
$$

Step 5: Proof of (5.5).

Set $f(\tau, x)=u(t-\tau, x) \bar{\phi}(t-\tau, x)$ for $\tau \in[0, t-a]$ and $g(\tau, x)=f_{B\left(x, \frac{\sqrt{b}}{2}\right)} f(\tau, y) \mathrm{d} y$. After averaging, we have to show that

$$
\lim _{\varepsilon \rightarrow 0} \int_{-\frac{1}{2}}^{\frac{1}{2}} \int_{\mathbb{R}^{n}} v(\sigma) g(2 \varepsilon(1-\sigma), x) \mathrm{d} x \mathrm{~d} \sigma=\int_{-\frac{1}{2}}^{\frac{1}{2}} \int_{\mathbb{R}^{n}} v(\sigma) g(0, x) \mathrm{d} x \mathrm{~d} \sigma .
$$

It follows from Proposition 3.6 and Proposition 3.9 that for all $x \in \mathbb{R}^{n}, f \in \mathscr{C}([0, t-$ $a] ; L^{1}\left(B\left(x, \frac{\sqrt{b}}{2}\right)\right)$. Hence $g(2 \varepsilon(1-\sigma), x) \rightarrow g(0, x)$ when $\varepsilon \rightarrow 0$ for all $(\sigma, x)$.

For $\tau=2 \varepsilon(1-\sigma)$, we have $\tau \in[0,3 \varepsilon] \subset[0, t-a]$. To apply dominated convergence, we show that $\sup _{\tau \in[0, t-a]}|g(\tau, x)|$ is integrable on $\mathbb{R}^{n}$. This is a variant of Step 1 to get 
uniformity. Indeed, for all $x \in \mathbb{R}^{n}$ and $\tau \in[0, t-a]$, by Proposition 3.6 and Proposition 3.19 with $\tau \leq b-a$

$$
\begin{aligned}
|g(\tau, x)| & \leq f_{B\left(x, \frac{\sqrt{b}}{2}\right)}|u(t-\tau, y)|\left|\Gamma(t, t-\tau)^{*} h(y)\right| \mathrm{d} y \\
& \lesssim\left(f_{B\left(x, \frac{\sqrt{b}}{2}\right)}|u(t-\tau, y)|^{2} \mathrm{~d} y\right)^{\frac{1}{2}}\left(\sum_{j=1}^{\infty}\left\|1_{B\left(x, \frac{\sqrt{b}}{2}\right)} \Gamma(t, t-\tau)^{*}\left(1_{S_{j}(x, \sqrt{b})} h\right)\right\|_{L^{2}}\right) \\
& \lesssim\left(\int_{a}^{b} \int_{B(x, \sqrt{b})}\left|u\left(\sigma^{\prime}, y\right)\right|^{2} \mathrm{~d} y \mathrm{~d} \sigma^{\prime}\right)^{\frac{1}{2}}\left(\sum_{j=1}^{\infty} e^{-\alpha \frac{4^{j} b}{b-a}}\left\|1_{S_{j}(x, \sqrt{b})} h\right\|_{L^{2}}\right) .
\end{aligned}
$$

This estimate is uniform with respect to $\tau$ and we get integrability as in Step 1 using Step 0.

Step 6: Proof of (5.6).

The proof is exactly the same as that of (5.5) taking now $f(\tau, x)=u(s-\tau, x) \bar{\phi}(s-\tau, x)$ for $\tau \in[0, s-a]$ and using $\tau=\varepsilon(1-2 \sigma)$.

\subsection{Results for $p \geq 2$}

Our uniqueness results will be based on the following well-know fact. Let $X$ be a Banach space and $Y$ its dual space. If $\left(y_{k}\right)_{k \in \mathbb{N}}$ is a sequence weakly* converging to $y$ in $Y$, and $\left(x_{k}\right)_{k \in \mathbb{N}}$ is a sequence strongly converging to $x$ in $X$, then $\left(\left\langle y_{k}, x_{k}\right\rangle\right)_{k \in \mathbb{N}}$ converges to $\langle y, x\rangle$. Of course, when $X$ is reflexive, weak* and weak convergence coincide.

We illustrate this principle by first proving that $L^{\infty}\left(L^{2}\right)$ is always a class of uniqueness for $L^{2}$ data. Next we look at $L^{p}$ data for $p>2$ using non-tangential maximal estimates.

Theorem 5.2. For $u \in \mathscr{D}^{\prime}$, the following assertions are equivalent.

$$
\begin{aligned}
& \exists ! f \in L^{2}\left(\mathbb{R}^{n}\right) \text { such that, for all } t>0, u(t, \cdot)=\Gamma(t, 0) f \text { in } L^{2}\left(\mathbb{R}^{n}\right) ; \\
& u \text { is a global weak solution of }(1.1) \text { in } L^{\infty}\left(L^{2}\right) .
\end{aligned}
$$

Proof. Proposition 3.14 gives us that (5.7) implies (5.8). We now assume (5.8), and note that $\sup _{t>0}\|u(t, \cdot)\|_{L^{2}}<\infty$ by Remark 4.5. Let $t>0$, and pick $\left(t_{k}\right)_{k \in \mathbb{N}}$ a decreasing sequence of real numbers converging to 0 , with $t_{0}=\frac{t}{2}$, such that there exists $f \in L^{2}\left(\mathbb{R}^{n}\right)$ with

$$
u\left(t_{k}, \cdot\right) \rightarrow f \quad \text { as } k \rightarrow \infty, \quad \text { weakly in } L^{2}\left(\mathbb{R}^{n}\right) .
$$

By Proposition 4.3, we can apply Theorem 5.1, and get that, for $k \in \mathbb{N}$ and $h \in \mathscr{C}_{c}\left(\mathbb{R}^{n}\right)$,

$$
\int_{\mathbb{R}^{n}} u(t, x) \overline{h(x)} \mathrm{d} x=\int_{\mathbb{R}^{n}} u\left(t_{k}, x\right) \overline{\Gamma\left(t, t_{k}\right)^{*} h(x)} \mathrm{d} x .
$$

By the continuity results in Proposition 3.17, we have that $\left\|\Gamma\left(t, t_{k}\right)^{*} h-\Gamma(t, 0)^{*} h\right\|_{2} \underset{k \rightarrow \infty}{\longrightarrow} 0$, and thus

$$
u(t, \cdot)=\Gamma(t, 0) f \quad \text { in } L^{2}\left(\mathbb{R}^{n}\right) .
$$

This also implies that $f=\lim _{t \rightarrow 0} u(t, \cdot)$ strongly in $L^{2}\left(\mathbb{R}^{n}\right)$, and proves the uniqueness of $f$.

The following corollary is an immediate consequence of Theorem 5.2 and Theorem 3.11. Recall from local estimates and Lions' result that a global weak solution of (1.1) in $L^{\infty}\left(L^{2}\right)$ is a priori in $\mathscr{C}\left([0,+\infty) ; L_{\text {loc }}^{2}\right)$. 
Corollary 5.3. For all $u_{0} \in L^{2}\left(\mathbb{R}^{n}\right)$, the problem

$$
\partial_{t} u=\operatorname{div} A \nabla u, \quad u \in L^{\infty}\left(L^{2}\right), \quad u(0, .)=u_{0},
$$

is well posed. Its solution u agrees with the energy solution, and, therefore, is such that

$$
\left\|u_{0}\right\|_{L^{2}}=\|u\|_{L^{\infty}\left(L^{2}\right)} \leq \sqrt{2 \Lambda}\|\nabla u\|_{L^{2}\left(L^{2}\right)} \leq \sqrt{\frac{\Lambda}{\lambda}}\left\|u_{0}\right\|_{L^{2}} .
$$

We now consider $p>2$.

Theorem 5.4. Let $p \in(2, \infty]$. For $u \in \mathscr{D}^{\prime}$, the following assertions are equivalent.

$$
\exists ! f \in L^{p}\left(\mathbb{R}^{n}\right) \text { such that, for all } t>0, u(t, \cdot)=\Gamma(t, 0) f \text { in } L_{\text {loc }}^{2}\left(\mathbb{R}^{n}\right) ;
$$

$u$ is a global weak solution of (1.1) with $\tilde{N}(u) \in L^{p}\left(\mathbb{R}^{n}\right)$.

Proof. Lemma 4.6 and Proposition 4.8 give us that $(5.9) \Longrightarrow(5.10)$. We now consider the other direction and assume that $\tilde{N}(u) \in L^{p}\left(\mathbb{R}^{n}\right)$. Since $p>2$, Lemma 2.4 and Proposition 3.6 yield, for all $0<t \leq \delta$ :

$$
\begin{aligned}
\|u(t, \cdot)\|_{E_{\delta}^{p}} & \lesssim\|u(t, \cdot)\|_{E_{\frac{t}{4}}^{p}}=\left(\int_{\mathbb{R}^{n}}\left(f_{B\left(x, \frac{\sqrt{t}}{2}\right)}|u(t, y)|^{2} \mathrm{~d} y\right)^{\frac{p}{2}} \mathrm{~d} x\right)^{\frac{1}{p}} \\
& \lesssim\left(\int_{\mathbb{R}^{n}}\left(f_{\frac{t}{2}}^{t} f_{B(x, \sqrt{t})}|u(s, y)|^{2} \mathrm{~d} y \mathrm{~d} s\right)^{\frac{p}{2}} \mathrm{~d} x\right)^{\frac{1}{p}} \leq\|\tilde{N}(u)\|_{p}=\|u\|_{X^{p}},
\end{aligned}
$$

the constants being independent of $t, \delta$ (with the usual modification if $p=\infty$ ). Fix $\delta>0$, and let $f_{\delta} \in E_{\delta}^{p}$, and $\left(t_{k}\right)_{k \in \mathbb{N}}$ be a decreasing sequence such that $t_{k} \underset{k \rightarrow \infty}{\longrightarrow} 0, t_{0}<\delta$ and

$$
u\left(t_{k}, \cdot\right) \stackrel{w_{e a k}^{*}}{\longrightarrow} f_{\delta} \quad \text { in } E_{\delta}^{p} .
$$

For each $j \geq 1$, as $E_{\frac{\delta}{j}}^{p}=E_{\delta}^{p}$ with equivalent norm, the weak* convergence holds in $E_{\frac{\delta}{j}}^{p}$ and $\left\|f_{\delta}\right\|_{E_{\frac{\delta}{j}}^{p}} \leq \liminf _{k \rightarrow \infty}\left\|u\left(t_{k}, .\right)\right\|_{E_{\frac{\delta}{j}}^{p}} \lesssim\|u\|_{X^{p}}$, the constant being independent of $j \geq 1$ and $\delta^{\frac{\delta}{j}}>0$. Therefore

$$
\left[x \mapsto\left(f_{B\left(x, \frac{\delta}{j}\right)}\left|f_{\delta}(y)\right|^{2} \mathrm{~d} y\right)^{\frac{1}{2}}\right] \in L^{p}\left(\mathbb{R}^{n}\right) \quad \forall j \geq 1 .
$$

Moreover

$$
\left(f_{B\left(x, \frac{\delta}{j}\right)}\left|f_{\delta}(y)\right|^{2} \mathrm{~d} y\right)^{\frac{1}{2}} \underset{j \rightarrow \infty}{\longrightarrow}\left|f_{\delta}(x)\right| \text { for a.e. } x \in \mathbb{R}^{n}
$$

by Lebesgue differentiation theorem. By Fatou's lemma, $f_{\delta} \in L^{p}\left(\mathbb{R}^{n}\right)$ and

$$
\left\|f_{\delta}\right\|_{p} \leq \liminf _{j \rightarrow \infty}\left\|f_{\delta}\right\|_{E_{\frac{\delta}{j}}^{p}} \lesssim\|u\|_{X^{p}}
$$

By Proposition 4.4, we can apply Theorem 5.1 to obtain, for all $k \in \mathbb{N}$ and $h \in \mathscr{C}_{c}\left(\mathbb{R}^{n}\right)$,

$$
\int_{\mathbb{R}^{n}} u\left(t_{k}, x\right) \overline{\Gamma\left(t, t_{k}\right)^{*} h(x)} \mathrm{d} x=\int_{\mathbb{R}^{n}} u(t, x) \overline{h(x)} \mathrm{d} x .
$$

Applying Lemma 4.7, we have that

$$
\Gamma\left(t, t_{k}\right)^{*} h \underset{k \rightarrow \infty}{\longrightarrow} \Gamma(t, 0)^{*} h \quad \text { in } E_{\delta}^{p^{\prime}} .
$$


Therefore $\int_{\mathbb{R}^{n}} u(t, x) \overline{h(x)} \mathrm{d} x=\int_{\mathbb{R}^{n}} f_{\delta}(x) \overline{\Gamma(t, 0)^{*} h(x)} \mathrm{d} x$ for all $t>0$ and all $h \in \mathscr{C}_{c}\left(\mathbb{R}^{n}\right)$, which gives us that $u(t, \cdot)=\Gamma(t, 0) f_{\delta}$ in $E_{\delta}^{p}$. This implies that $f_{\delta}=\lim _{t \rightarrow 0} u(t, \cdot)$ strongly in $E_{\delta}^{p}$ for $p<\infty$ and in $L_{\text {loc }}^{2}$ for $p=\infty$ by Lemma 4.7. Therefore, $f_{\delta}=\lim _{t \rightarrow 0} u(t, \cdot)$ strongly in $L_{\mathrm{loc}}^{2}$ in all cases and $f_{\delta}$ is independent of $\delta$. We write $f=f_{\delta}$. This $f$ is unique as $\lim _{t \rightarrow 0} u(t, \cdot)$ in $L_{\text {loc }}^{2}$, and $f \in L^{p}\left(\mathbb{R}^{n}\right)$ with $\|f\|_{p} \lesssim\|\tilde{N}(u)\|_{p}$ as proven above.

The following corollary is now immediate.

Corollary 5.5. Let $p \in(2, \infty]$ and $u_{0} \in L^{p}\left(\mathbb{R}^{n}\right)$. There exists a unique global weak solution $u$ of (1.1) in $X^{p}$ such that $\lim _{t \rightarrow 0} u(t, \cdot)=u_{0}$ in $L_{\mathrm{loc}}^{2}$. Moreover, $\|u\|_{X^{p}} \sim\left\|u_{0}\right\|_{L^{p}}$.

An interesting consequence of our result in $X^{\infty}$ is the following conservation property of the propagators.

Corollary 5.6. Let $t>s$. Then

$$
\Gamma(t, s) 11=11 \text { in } L_{\mathrm{loc}}^{2}\left(\mathbb{R}^{n}\right) .
$$

Similarly

$$
\Gamma(t, s)^{*} 11=11 \text { in } L_{\mathrm{loc}}^{2}\left(\mathbb{R}^{n}\right) .
$$

Proof. We may assume $s=0$ without loss of generality The constant function 11 on $\mathbb{R}_{+}^{n+1}$ is a global weak solution of (1.1) and belongs to $X^{\infty}$. By Theorem 5.4, we have that, for almost every $(t, x) \in \mathbb{R}_{+}^{n+1}, \mathbb{1}=\Gamma(t, 0) f(x)$ for a unique $f \in L^{\infty}$ such that $f=\lim _{t \rightarrow 0} \Gamma(t, 0) f$ in $L_{\text {loc }}^{2}\left(\mathbb{R}^{n}\right)$. Thus, $f=1$ almost everywhere on $\mathbb{R}^{n}$ and we have shown the equality in $L_{\text {loc }}^{2}\left(\mathbb{R}_{+}^{n+1}\right)$. As weak solutions are continuous in time with values in $L_{\text {loc }}^{2}\left(\mathbb{R}^{n}\right)$, the conclusion follows. The formula for the adjoint is obtained similarly using that we get the same $X^{\infty}$ result for the backward equation on $(-\infty, t)$. See Remark 3.18.

We finish with a result valid in full generality, getting closer to $L^{p}$ estimates.

Proposition 5.7. Let $\tilde{q}>2+\frac{4}{n}$ be the exponent in the reverse Hölder estimates of Corollary 4.2. Fix $p \in(2, \tilde{q})$. For $u \in \mathscr{D}^{\prime}$, the following assertions are equivalent.

$$
\begin{aligned}
& \exists ! f \in L^{p}\left(\mathbb{R}^{n}\right) \text { such that, for all } t>0, u(t, \cdot)=\Gamma(t, 0) f \text { in } L_{\mathrm{loc}}^{2}\left(\mathbb{R}^{n}\right) ; \\
& u \text { is a global weak solution of (1.1) with } \sup _{a>0}\left\|f_{a}^{2 a}|u(t, \cdot)| \mathrm{d} t\right\|_{L^{p}}<\infty .
\end{aligned}
$$

In this case, $\|f\|_{L^{p}} \sim \sup _{a>0}\left\|f_{a}^{2 a}|u(t, \cdot)| \mathrm{d} t\right\|_{L^{p}}$ and $f_{a}^{2 a} u(t, \cdot) \mathrm{d} t$ converges to $f$ in $L^{p}\left(\mathbb{R}^{n}\right)$ as $a \rightarrow 0$.

Proof. For the direct part, let $f \in L^{p}\left(\mathbb{R}^{n}\right)$ and $u(t, \cdot)=\Gamma(t, 0) f$. By Theorem 5.4, we know that $u$ is a global weak solution and that $u \in X^{p}$. Using the reverse Hölder estimates of Corollary 4.2, we see that we may replace the $L^{2}$ averages by $L^{p}$ averages in the definition of $\tilde{N}(u)$ (up to modifying slightly the parameters). Hence by Hölder's inequality and averaging

$$
\left\|f_{a}^{2 a}|u(t, \cdot)| \mathrm{d} t\right\|_{L^{p}}^{p} \leq \int_{\mathbb{R}^{n}} f_{a}^{2 a}|u(t, x)|^{p} \mathrm{~d} t \mathrm{~d} x \lesssim\|\tilde{N}(u)\|_{L^{p}}^{p} .
$$


This proves the direct part. In addition, this implies that $f_{a}^{2 a} \Gamma(t, 0) \mathrm{d} t$ are bounded operators on $L^{p}\left(\mathbb{R}^{n}\right)$ uniformly with respect to $a$. This is true for all $p \in[2, \tilde{q})$. At the same time, they converge strongly in $\mathcal{L}\left(L^{2}\right)$ to $I$ when $a \rightarrow 0$. By an interpolation argument (see the proof of the next result, Theorem 5.9) this implies the strong continuity at 0 in $\mathscr{L}\left(L^{p}\right)$. In particular, this yields the norm comparison in the statement.

Let us now prove the converse and assume that $u$ is a global weak solution of (1.1) with $M=\sup _{a>0}\left\|f_{a}^{2 a}|u(t, \cdot)| \mathrm{d} t\right\|_{L^{p}}<\infty$. For all $\delta>0$ and $t \leq \delta$, using Lemma 2.4 with $p>2$, Proposition 3.6 and the reverse Hölder estimate of Corollary 4.2 again, we have

$$
\begin{aligned}
\|u(t, \cdot)\|_{E_{\delta}^{p}} & \lesssim\|u(t, \cdot)\|_{E_{\frac{t}{32}}^{p}}=\left(\int_{\mathbb{R}^{n}}\left(f_{B\left(x, \sqrt{\frac{t}{32}}\right)}|u(t, y)|^{2} \mathrm{~d} y\right)^{\frac{p}{2}} \mathrm{~d} x\right)^{\frac{1}{p}} \\
& \lesssim\left(\int_{\mathbb{R}^{n}}\left(f_{\frac{7 t}{8}}^{\frac{9 t}{8}} f_{B\left(x, \sqrt{\frac{t}{8}}\right)}|u(s, y)|^{2} \mathrm{~d} y \mathrm{~d} s\right)^{\frac{p}{2}} \mathrm{~d} x\right)^{\frac{1}{p}} \\
& \lesssim\left(\int_{\mathbb{R}^{n}}\left(f_{\frac{t}{2}}^{t} f_{B\left(x, \sqrt{\frac{t}{2}}\right)}|u(s, y)| \mathrm{d} y \mathrm{~d} s\right)^{p} \mathrm{~d} x\right)^{\frac{1}{p}} \\
& =\left(\int_{\mathbb{R}^{n}}\left(f_{B\left(x, \sqrt{\frac{t}{2}}\right)} f_{\frac{t}{2}}^{t}|u(s, y)| \mathrm{d} s \mathrm{~d} y\right)^{p} \mathrm{~d} x\right)^{\frac{1}{p}} \\
& \leq\left(\int_{\mathbb{R}^{n}} f_{B\left(x, \sqrt{\frac{t}{2}}\right)}\left(f_{\frac{t}{2}}^{t}|u(s, y)| \mathrm{d} s\right)^{p} \mathrm{~d} y \mathrm{~d} x\right)^{\frac{1}{p}} \leq M .
\end{aligned}
$$

Thus we have the uniform estimate in the slice space $E_{\delta}^{p}$ as in the proof of Theorem 5.4 and the same argument applies. This proves the converse.

Remark 5.8. In the previous theorem, $u$ has further regularity: $\left(f_{a}^{2 a}|u(t, \cdot)|^{p} \mathrm{~d} t\right)^{\frac{1}{p}} \in$ $L^{p}\left(\mathbb{R}^{n}\right)$ uniformly in $a>0$ and $\|f\|_{L^{p}} \sim \sup _{a>0}\left\|\left(f_{a}^{2 a}|u(t, \cdot)|^{p} \mathrm{~d} t\right)^{\frac{1}{p}}\right\|_{L^{p}}$ as one can check. The largest class in this scale for uniqueness is the one in the statement.

\subsection{Results for $p<2$}

For $p<2$ we do not know general results without imposing further properties of the propagators. Here we assume boundedness of the propagators acting on $L^{p}$, and consider solutions in $L^{\infty}\left(L^{p}\right)$. Note that, by Remarks 4.5 and 4.12 , we can assume uniform boundedness rather than almost everywhere boundedness.

Theorem 5.9. Let $1 \leq q<p<2$. Assume that $\sup _{0 \leq s \leq t<\infty}\|\Gamma(t, s)\|_{\mathscr{L}\left(L^{q}\right)}<\infty$. Let $u \in$ $L^{\infty}\left((0, \infty) ; L^{p}\left(\mathbb{R}^{n}\right)\right)$ be a global weak solution of (1.1). Then there exists $u_{0} \in L^{p}\left(\mathbb{R}^{n}\right)$ such that $u(t, \cdot)=\Gamma(t, 0) u_{0}$ in $L^{p}\left(\mathbb{R}^{n}\right)$ for all $t>0$. Moreover, $u \in \mathscr{C}_{0}\left([0, \infty) ; L^{p}\left(\mathbb{R}^{n}\right)\right)$ and, in particular, $u_{0}$ is unique.

Proof. Let $u \in L^{\infty}\left(L^{p}\right)$ be a global weak solution of (1.1). Let $t>0$, and pick $\left(t_{k}\right)_{k \in \mathbb{N}}$ a decreasing sequence of real numbers converging to 0 , with $t_{0}<\frac{t}{2}$, such that there exists $u_{0} \in L^{p}\left(\mathbb{R}^{n}\right)$ with

$$
u\left(t_{k}, \cdot\right) \rightarrow u_{0} \quad \text { as } k \rightarrow \infty, \quad \text { weakly* in } L^{p}\left(\mathbb{R}^{n}\right) .
$$


By Proposition 4.3 and Theorem 5.1, for $k \in \mathbb{N}$ and $h \in \mathscr{C}_{c}\left(\mathbb{R}^{n}\right)$, we have that

$$
\int_{\mathbb{R}^{n}} u(t, x) \overline{h(x)} \mathrm{d} x=\int_{\mathbb{R}^{n}} u\left(t_{k}, x\right) \overline{\Gamma\left(t, t_{k}\right)^{*} h(x)} \mathrm{d} x .
$$

It remains to prove that $\left\|\Gamma\left(t, t_{k}\right)^{*} h-\Gamma(t, 0)^{*} h\right\|_{p^{\prime}} \underset{k \rightarrow \infty}{\longrightarrow} 0$. By Lemma 4.9, and the fact that $t-t_{k} \sim t$ for all $k \in \mathbb{N}$, we have that $\sup _{k \in \mathbb{N}}\left\|\Gamma\left(t, t_{k}\right)^{*}\right\|_{\mathscr{L}\left(L^{2}, L^{r^{\prime}}\right)}<\infty$ for all $r^{\prime} \in\left(p^{\prime}, q^{\prime}\right)$. Let $\theta \in[0,1)$ be defined by $\frac{1}{p^{\prime}}=\frac{\theta}{r^{\prime}}+\frac{1-\theta}{2}$. For all $h \in \mathscr{C}_{c}^{\infty}\left(\mathbb{R}^{n}\right)$,

$$
\begin{aligned}
\left\|\Gamma\left(t, t_{k}\right)^{*} h-\Gamma(t, 0)^{*} h\right\|_{L^{p^{\prime}}} & \leq\left\|\Gamma\left(t, t_{k}\right)^{*} h-\Gamma(t, 0)^{*} h\right\|_{L^{r^{\prime}}}^{\theta}\left\|\Gamma\left(t, t_{k}\right)^{*} h-\Gamma(t, 0)^{*} h\right\|_{L^{2}}^{1-\theta} \\
& \lesssim\left\|\Gamma\left(t, t_{k}\right)^{*} h-\Gamma(t, 0)^{*} h\right\|_{L^{2}}^{1-\theta} \underset{k \rightarrow \infty}{\longrightarrow} 0 .
\end{aligned}
$$

We now show that $u \in \mathscr{C}\left([0, \infty) ; L^{p}\left(\mathbb{R}^{n}\right)\right)$. Let $\varepsilon>0$ and $v_{0} \in \mathscr{C}_{c}^{\infty}\left(\mathbb{R}^{n}\right)$ be such that $\left\|u_{0}-v_{0}\right\|_{L^{p}}<\varepsilon$. Let $s, t>0$ :

$$
\begin{aligned}
\left\|\Gamma(t, 0) u_{0}-\Gamma(s, 0) u_{0}\right\|_{L^{p}} & \leq\left\|\Gamma(t, 0)\left(u_{0}-v_{0}\right)\right\|_{L^{p}}+\left\|\Gamma(t, 0) v_{0}-\Gamma(s, 0) v_{0}\right\|_{L^{p}}+\left\|\Gamma(s, 0)\left(v_{0}-u_{0}\right)\right\|_{L^{p}} \\
& \lesssim \varepsilon+\left\|\Gamma(t, 0) v_{0}-\Gamma(s, 0) v_{0}\right\|_{L^{q}}^{\theta}\left\|\Gamma(t, 0) v_{0}-\Gamma(s, 0) v_{0}\right\|_{L^{2}}^{1-\theta}
\end{aligned}
$$

for $\theta \in(0,1]$ such that $\frac{1}{p}=\frac{\theta}{q}+\frac{1-\theta}{2}$. Therefore

$$
\left\|\Gamma(t, 0) u_{0}-\Gamma(s, 0) u_{0}\right\|_{L^{p}} \lesssim \varepsilon+\left\|v_{0}\right\|_{L^{q}}^{\theta}\left\|\Gamma(t, 0) v_{0}-\Gamma(s, 0) v_{0}\right\|_{L^{2}}^{1-\theta} .
$$

Since $\left(t \mapsto \Gamma(t, 0) v_{0}\right) \in \mathscr{C}\left([0, \infty) ; L^{2}\left(\mathbb{R}^{n}\right)\right)$, there exists $\delta>0$ such that for all $t, s>0$ with $|t-s|<\delta,\left\|\Gamma(t, 0) v_{0}-\Gamma(s, 0) v_{0}\right\|_{L^{2}} \leq\left(\frac{\varepsilon}{\left\|v_{0}\right\|_{L^{q}}^{\theta}}\right)^{\frac{1}{1-\theta}}$. This proves that

$$
\left\|\Gamma(t, 0) u_{0}-\Gamma(s, 0) u_{0}\right\|_{L^{p}} \lesssim \varepsilon \quad \forall t, s>0,|t-s|<\delta,
$$

and then the fact that $\left(t \mapsto \Gamma(t, 0) u_{0}\right)$ is continuous in $L^{p}\left(\mathbb{R}^{n}\right)$. In particular,

$$
u_{0}=\lim _{t \rightarrow 0} \Gamma(t, 0) u_{0}=\lim _{t \rightarrow 0} u(t, \cdot) .
$$

Since we know moreover that $\left(t \mapsto \Gamma(t, 0) v_{0}\right) \in \mathscr{C}_{0}\left([0, \infty) ; L^{2}\left(\mathbb{R}^{n}\right)\right)$, the same reasoning shows that $\left\|\Gamma(t, 0) u_{0}\right\|_{L^{p}} \underset{t \rightarrow \infty}{\longrightarrow} 0$.

Corollary 5.10. Let $1 \leq q<p<2$. Assume that $\sup _{0 \leq s \leq t<\infty}\|\Gamma(t, s)\|_{\mathscr{L}\left(L^{q}\right)}<\infty$. For $u \in \mathscr{D}^{\prime}$, the following assertions are equivalent.

$u$ is a global weak solution of $(1.1)$ in $L^{\infty}\left((0, \infty) ; L^{p}\left(\mathbb{R}^{n}\right)\right)$;

$\exists ! u_{0} \in L^{p}\left(\mathbb{R}^{n}\right)$ such that $u(t, \cdot)=\Gamma(t, 0) u_{0}$ in $L^{p}\left(\mathbb{R}^{n}\right)$ for all $t>0 ;$

$u$ is a global weak solution of (1.1) in $X^{p}$.

In this case, $u \in \mathscr{C}_{0}\left(L^{p}\right)$ and $\left\|u_{0}\right\|_{p} \sim\|u\|_{L^{\infty}\left(L^{p}\right)} \sim\|u\|_{X^{p}}$. 
Proof. (5.13) $\Longrightarrow(5.14)$ is proven in Theorem 5.9. The implication (5.14) $\Longrightarrow(5.13)$ is a consequence of $\sup _{0 \leq s \leq t<\infty}\|\Gamma(t, s)\|_{\mathscr{L}\left(L^{p}\right)}<\infty$, and Lemma 4.10. So is the norm estimate $\left\|u_{0}\right\|_{p} \sim\|u\|_{L^{\infty}\left(L^{p}\right)}$.

$(5.15) \Longrightarrow(5.13)$ : Let $t>0$. Using Proposition 3.6 and Hölder's inequality, we have that

$$
\begin{aligned}
\|u(t, \cdot)\|_{p} & =\left(\int_{\mathbb{R}^{n}} f_{B\left(x, \frac{\sqrt{t}}{2}\right)}|u(t, y)|^{p} \mathrm{~d} y \mathrm{~d} x\right)^{\frac{1}{p}} \\
& \lesssim\left(\int_{\mathbb{R}^{n}}\left(f_{B\left(x, \frac{\sqrt{t}}{2}\right)}|u(t, y)|^{2} \mathrm{~d} y\right)^{\frac{p}{2}} \mathrm{~d} x\right)^{\frac{1}{p}} \\
& \lesssim\left(\int_{\mathbb{R}^{n}}\left(f_{\frac{t}{2}}^{t} f_{B(x, \sqrt{t})}|u(\sigma, y)|^{2} \mathrm{~d} y \mathrm{~d} \sigma\right)^{\frac{p}{2}} \mathrm{~d} x\right)^{\frac{1}{p}} \leq\|\tilde{N}(u)\|_{p} .
\end{aligned}
$$

(5.14) $\Longrightarrow$ (5.15): Let $r \in(q, p)$, and $x \in \mathbb{R}^{n}, \delta>0$. Using Lemma 4.9 we have that

$$
\left(f_{\frac{\delta}{2}}^{\delta} f_{B(x, \sqrt{\delta})}\left|\Gamma(t, 0) u_{0}(y)\right|^{2} \mathrm{~d} y \mathrm{~d} t\right)^{\frac{1}{2}} \lesssim\left(M_{H L}\left|u_{0}\right|^{r}(x)\right)^{\frac{1}{r}}
$$

with constants independent of $x, \delta$. Therefore $\|\tilde{N}(u)\|_{L^{p}} \lesssim\left\|u_{0}\right\|_{L^{p}} \leq\|u\|_{L^{\infty}\left(L^{p}\right)}$ as we have shown in the proof of Theorem 5.9 that $t \mapsto \Gamma(t, 0) u_{0}$ is continuous in $L^{p}\left(\mathbb{R}^{n}\right)$. Moreover $u$ is a global weak solution of (1.1) by Lemma 4.10.

\subsection{Further results}

Without any assumption on the propagators, we have proven well posedness results in the class $X^{p}$ for $p>2$. We now consider solutions in $L^{\infty}\left(L^{p}\right)$ under an $L^{p}$ boundedness assumption on the propagators. Note that, contrary to the case $p<2$, we do not need to make assumptions about the boundedness of the propagators for different values of $p$.

Proposition 5.11. Let $p \in(2, \infty]$. Assume that $\sup _{0 \leq s \leq t<\infty}\|\Gamma(t, s)\|_{\mathscr{L}\left(L^{p}\right)}<\infty$. For $u \in \mathscr{D}^{\prime}$, the following assertions are equivalent.

$u$ is a global weak solution of (1.1) in $L^{\infty}\left(L^{p}\right)$;

$\exists ! u_{0} \in L^{p}\left(\mathbb{R}^{n}\right)$ such that $u(t, \cdot)=\Gamma(t, 0) u_{0}$ in $L^{p}\left(\mathbb{R}^{n}\right)$ for all $t>0$.

In this case, $\left\|u_{0}\right\|_{p} \sim\|u\|_{L^{\infty}\left(L^{p}\right)} \sim\|u\|_{X^{p}}$.

Moreover, if $p<\infty$ and $\sup _{0 \leq s \leq t<\infty}\|\Gamma(t, s)\|_{\mathscr{L}\left(L^{r}\right)}<\infty$ for some $r \in(p, \infty)$ then $u \in \mathscr{C}_{0}\left(L^{p}\right)$.

Proof. Proposition 4.8 and the assumption give us that (5.17) implies (5.16), with $\left\|u_{0}\right\|_{L^{p}} \sim$ $\|u\|_{L^{\infty}\left(L^{p}\right)}$. We now prove that (5.16) implies (5.17). Proceeding as in the proof of Theorem 5.9 , we only have to show that

$$
\left\|\Gamma(t, s)^{*} h-\Gamma(t, 0)^{*} h\right\|_{L^{p^{\prime}}} \underset{s \rightarrow 0}{\longrightarrow} 0
$$

for all $t>0$ and $h \in \mathscr{C}_{c}\left(\mathbb{R}^{n}\right)$. Let $M>0$ be such that $h$ is supported in $B(0, M)$. For all $j \geq 1$ and $t>s>0$, we have that

$$
\left\|1_{S_{j}(0, M)}\left(\Gamma(t, s)^{*}-\Gamma(t, 0)^{*}\right) h\right\|_{L^{p^{\prime}}} \lesssim_{M} 2^{j n\left(\frac{1}{2}-\frac{1}{p}\right)}\left\|1_{S_{j}(0, M)}\left(\Gamma(t, s)^{*}-\Gamma(t, 0)^{*}\right) h\right\|_{L^{2}} .
$$


For each $j \geq 1$, the right hand side converges to 0 when $s \rightarrow 0$ by strong continuity of $s \mapsto \Gamma(t, s)^{*} h$ by Proposition 3.17. Combining this estimate with Proposition 3.19, we have the following for all $j \geq 2$, and some constant $c>0$ :

$$
\left\|1_{S_{j}(0, M)}\left(\Gamma(t, s)^{*}-\Gamma(t, 0)^{*}\right) h\right\|_{L^{p^{\prime}}} \lesssim M 2^{j n\left(\frac{1}{2}-\frac{1}{p}\right)} e^{-c \frac{4^{j}}{t}}\|h\|_{L^{2}},
$$

with constant independent of $s$ when $s<t / 2$. Therefore, we can apply dominated convergence for sums to obtain

$$
\left\|\Gamma(t, s)^{*} h-\Gamma(t, 0)^{*} h\right\|_{L^{p^{\prime}}} \leq \sum_{j \geq 1}\left\|1_{S_{j}(0, M)}\left(\Gamma(t, s)^{*}-\Gamma(t, 0)^{*}\right) h\right\|_{L^{p^{\prime}}} \underset{s \rightarrow 0}{\longrightarrow} 0 .
$$

The uniqueness of $u_{0}$ follows from convergence in $L_{\text {loc }}^{2}$ of $u$, since we know that $u(t, \cdot)=$ $\Gamma(t, 0) u_{0}$ for all $t>0$. The equivalence of norms follows from the above and Corollary 5.5.

If we assume that $p<\infty$ and that $\sup _{0<s<t<\infty}\|\Gamma(t, s)\|_{\mathscr{L}\left(L^{r}\right)}<\infty$ for some $r \in(p, \infty)$, then we obtain that $u \in \mathscr{C}_{0}\left(L^{p}\right)$ exactly as in the proof of Theorem 5.9.

An interesting corollary is the following weak maximum principle without continuity.

Corollary 5.12. Assume that $C=\sup _{0 \leq s \leq t<\infty}\|\Gamma(t, s)\|_{\mathscr{L}\left(L^{\infty}\right)}<\infty$. Then any global weak solution $u$ of (1.1) in $L^{\infty}\left(\mathbb{R}_{+}^{n+1}\right)$ satisfies

$$
\sup _{t>0}\|u(t, \cdot)\|_{L^{\infty}\left(\mathbb{R}^{n}\right)} \leq C\|f\|_{L^{\infty}\left(\mathbb{R}^{n}\right)},
$$

where $f$ is the initial value of $u$ (which exists as limit in the $L_{\mathrm{loc}}^{2}$ sense).

We end this section with another corollary assuming pointwise bounds. Remark that this does not include $p=1$.

Corollary 5.13. Assume the propagators $\Gamma(t, s), 0 \leq s<t<\infty$, have kernels bounds. Let $1<p \leq \infty$. For $u \in \mathscr{D}^{\prime}$, the following assertions are equivalent.

$u$ is a global weak solution of (1.1) in $L^{\infty}\left(L^{p}\right)$;

$\exists ! u_{0} \in L^{p}\left(\mathbb{R}^{n}\right)$ such that $u(t, \cdot)=\Gamma(t, 0) u_{0}$ in $L^{p}\left(\mathbb{R}^{n}\right)$ for all $t>0 ;$

$u$ is a global weak solution of (1.1) such that $\tilde{u} \in L^{p}\left(\mathbb{R}^{n}\right)$,

where

$$
\tilde{u}(x)=\sup _{t>0} \operatorname{esssup}_{y ;|y-x|<4 \sqrt{t}}|u(t, y)|, \quad x \in \mathbb{R}^{n} .
$$

In this case, $u \in \mathscr{C}_{0}\left(L^{p}\right)$ and $\left\|u_{0}\right\|_{p} \sim\|u\|_{L^{\infty}\left(L^{p}\right)} \sim\|\tilde{u}\|_{p} \sim\|u\|_{X^{p}}$.

Recall that solutions have no reason to be defined at each point, hence the variant of the pointwise maximal function.

Proof. As mentioned, $\Gamma(t, s)$ extends to uniformly bounded operators on $L^{p}$ when $t \geq s>0$. Corollary 5.10 and Proposition 5.11 thus yield the result, at least for the modified nontangential maximal function $\tilde{N}(u)$ instead of the standard non-tangential maximal function $u^{*}$. However, $\|\tilde{N}(u)\|_{L^{p}} \sim\|\tilde{u}\|_{L^{p}}$. Indeed, we first observe that $\tilde{N}(u) \leq \tilde{u}$. A converse inequality $\tilde{u} \lesssim \tilde{N}_{\beta}(u)$, for some $\beta>0$, follows from the local boundedness properties of solutions as stated in Proposition 4.13. Since $\left\|\tilde{N}_{\beta}(u)\right\|_{L^{p}} \sim\|\tilde{N}(u)\|_{L^{p}}$, the proof is complete. 


\section{Close to constant or bounded variation time dependency}

In this section, we obtain well-posedness results for $L^{p}$ data when $p<2$. It seems to us that one should be able to extend the following results to $p>2$ but this would require other methods and we leave this open.

\subsection{More about gradient bounds for semigroups}

We need to use the following quantified version of the boundedness property for the gradient of semigroups for autonomous problems.

Definition 6.1. For $1 \leq q<2, \Lambda, \lambda>0, M:\left[2, q^{\prime}\right) \rightarrow(0, \infty)$, let us define $\mathcal{M}(\Lambda, \lambda, q, M) \subset$ $L^{\infty}\left(\mathbb{R}^{n} ; \mathscr{M}_{n}(\mathbb{C})\right)$ by $A \in \mathcal{M}(\Lambda, \lambda, q, M)$ if and only if $A$ satisfies (1.2) with constants $\Lambda, \lambda$, and the following holds for $L=-\operatorname{div} A \nabla$,

$$
\sup _{t>0}\left\|\sqrt{t} \nabla e^{-t L^{*}}\right\|_{\mathscr{L}\left(L^{r}\right)} \leq M(r)<\infty \quad \forall r \in\left[2, q^{\prime}\right) .
$$

As mentioned in the proof of Proposition 2.8, this implies that there exists a function $M^{\prime}:[1,2] \cap\left(\frac{n q}{n+q}, 2\right] \rightarrow(0, \infty)$, such that

$$
\sup _{t \geq 0}\left\|e^{-t L}\right\|_{\mathscr{L}\left(L^{p}\right)} \leq M^{\prime}(p)<\infty \quad \forall p \in[1,2] \cap\left(\frac{n q}{n+q}, 2\right]
$$

Recall that for $p=2, M^{\prime}(2)=1$ by the contraction property ot the semigroup.

Remark 6.2. Any $A$ constant, or even continuous and periodic or almost periodic on $\mathbb{R}^{n}$ belongs to $\mathcal{M}(\Lambda, \lambda, 1, M)$ for some function $M$ (see [5, Section 3] and the references therein).

Definition 6.3. Let $A \in L^{\infty}\left(\mathbb{R}_{+}^{n+1} ; \mathscr{M}_{n}(\mathbb{C})\right)$ and $I \subset \mathbb{R}_{+}$be a bounded interval. We define $A_{I}=f_{I} A(t,.) \mathrm{d} t \in L^{\infty}\left(\mathbb{R}^{n} ; \mathscr{M}_{n}(\mathbb{C})\right)$.

Lemma 6.4. If $A \in L^{\infty}\left(\mathbb{R}_{+}^{n+1} ; \mathscr{M}_{n}(\mathbb{C})\right)$ satisfies (1.2), then there exist $q \in[1,2)$, and $M:\left[2, q^{\prime}\right) \rightarrow(0, \infty)$ such that

$$
A_{I} \in \mathscr{M}(\Lambda, \lambda, q, M) \text { for all bounded interval } I .
$$

Proof. It is immediate that $A_{I}$ satisfies (1.2) with constants $\Lambda, \lambda$. We need the existence of $q$ and $M$ that works for all $A_{I}$. This is provided by Remark 2.11.

\subsection{Existence and uniqueness for $p<2$ with $B V\left(L^{\infty}\right)$ coefficients}

Definition 6.5. We denote by $B V\left(L^{\infty}\right):=B V\left([0, \infty) ; L^{\infty}\left(\mathbb{R}^{n} ; \mathscr{M}_{n}(\mathbb{C})\right)\right)$ the space of functions $A:(0, \infty) \rightarrow L^{\infty}\left(\mathbb{R}^{n} ; \mathscr{M}_{n}(\mathbb{C})\right)$ with (semi-)norm

$$
\|A\|_{B V\left(L^{\infty}\right)}=\sup \left\{\sum_{k=0}^{\infty}\left\|A\left(t_{k+1}, \cdot\right)-A\left(t_{k}, \cdot\right)\right\|_{L^{\infty}} ;\left(t_{k}\right)_{k \in \mathbb{N}} \text { non decreasing in }[0, \infty)\right\} .
$$

If the semi-norm is zero then $A$ is independent of $t$. The $B V$ condition can thus be seen as a (large) perturbation of the autonomous case.

Let

$$
A(t, x)=\sum_{k=0}^{\infty} \mathbb{1}_{\left[t_{k}, t_{k+1}\right)}(t) A_{k}(x)
$$


with $A_{k} \in L^{\infty}\left(\mathbb{R}^{n} ; \mathscr{M}_{n}(\mathbb{C})\right)$ for all $k \in \mathbb{N}$, and $\left(t_{k}\right)_{k \in \mathbb{N}}$ increasing from $t_{0}=0$ to $\infty$. It is easy to see that $A \in B V\left(L^{\infty}\right)$ if and only if $\sum_{k=0}^{\infty}\left\|A_{k+1}-A_{k}\right\|_{L^{\infty}}<\infty$, and in this case the sum equals $\|A\|_{B V\left(L^{\infty}\right)}$. Moreover, if all $A_{k}$ satisfy (1.2) with same ellipticity constants $\lambda, \Lambda$, then so does $A$. This is representative of the general situation thanks to the next lemma.

Lemma 6.6. Let $A \in L^{\infty}\left(\mathbb{R}_{+}^{n+1} ; \mathscr{M}_{n}(\mathbb{C})\right) \cap B V\left(L^{\infty}\right)$ satisfy (1.2) with constants $\Lambda, \lambda$. For $j \in \mathbb{N}$, and $(t, x) \in \mathbb{R}_{+}^{n+1}$, let us define

$$
A_{j}(t, x)=\sum_{m=0}^{\infty} \mathbb{1}_{\left[\frac{m}{2^{j}}, \frac{m+1}{2^{j}}\right)}(t) f_{\frac{m}{2^{j}}}^{\frac{m+1}{2^{j}}} A(s, x) \mathrm{d} s .
$$

Then

(i) For all $j \in \mathbb{N}, A_{j}$ satisfies (1.2) with constants $\Lambda, \lambda$.

(ii) For almost every $(t, x) \in(0, \infty) \times \mathbb{R}^{n}, A_{j}(t, x) \underset{j \rightarrow \infty}{\longrightarrow} A(t, x)$.

(iii) For all $j \in \mathbb{N},\left\|A_{j}\right\|_{B V\left(L^{\infty}\right)} \leq\|A\|_{B V\left(L^{\infty}\right)}$.

Proof. $(i)$ and $(i i)$ follow directly from the definition of $A_{j}$ and Lebesgue's differentiation theorem. We turn to (iii). By the discussion above

$$
\begin{aligned}
\left\|A_{j}\right\|_{B V\left(L^{\infty}\right)} & =\sum_{m=0}^{\infty}\left\|f_{\frac{m+1}{2^{j}}}^{\frac{m+2}{2^{j}}} A(s, x) d s-f_{\frac{m}{2^{j}}}^{\frac{m+1}{2^{j}}} A(s, x) d s\right\|_{L^{\infty}} \\
& \leq f_{0}^{2^{-j}} \sum_{m=0}^{\infty}\left\|A\left(\frac{m+1}{2^{j}}+s, \cdot\right)-A\left(\frac{m}{2^{j}}+s, \cdot\right)\right\|_{L^{\infty}} \mathrm{d} s \leq\|A\|_{B V\left(L^{\infty}\right)} .
\end{aligned}
$$

Lemma 6.7. Let $q \in[1,2), M:\left[2, q^{\prime}\right) \rightarrow(0, \infty)$, and $\Lambda, \lambda>0$. Let $A \in L^{\infty}\left(\mathbb{R}_{+}^{n+1} ; \mathscr{M}_{n}(\mathbb{C})\right) \cap$ $B V\left(L^{\infty}\right)$ be of the form

$$
A(t, x)=\sum_{k=0}^{\infty} \mathbb{1}_{\left[t_{k}, t_{k+1}\right)}(t) A_{k}(x)
$$

with $A_{k} \in \mathcal{M}(\Lambda, \lambda, q, M)$ for all $k \in \mathbb{N}$, and $\left(t_{k}\right)_{k \in \mathbb{N}}$ increasing from $t_{0}=0$ to $\infty$. Then, for all $p \in\left(\max \left\{\left(1, \frac{2 n}{n+q^{\prime}}\right\}, 2\right)\right.$, and $v_{0} \in L^{p}\left(\mathbb{R}^{n}\right)$,

$$
\left[v:(t, x) \mapsto \Gamma(t, 0) v_{0}(x)\right] \in L^{\infty}\left(L^{p}\right)
$$

and $\left\|v_{0}\right\|_{p} \sim\|v\|_{L^{\infty}\left(L^{p}\right)}$, with constants depending only on $p, q, M, \lambda, \Lambda$ and the $B V$ norm of $A$.

Remark 6.8. The range of $p$ within $[1,2)$ depends only on the one of Proposition 2.8. According to Remark 2.10, $\frac{2 n}{n+q^{\prime}}$ can be improved to $\frac{q n}{n+q}$.

Proof. By density, it is enough to assume $v_{0} \in L^{2}\left(\mathbb{R}^{n}\right) \cap L^{p}\left(\mathbb{R}^{n}\right)$. Then $v \in \mathscr{C}_{0}\left(L^{2}\right)$. For $k \in \mathbb{N}$, set $v_{k}=\Gamma\left(t_{k}, 0\right) v_{0}$ and

$$
w_{k}(t, \cdot)= \begin{cases}e^{-\left(t-t_{k}\right) L_{k}} v_{k}, & \text { if } t \geq t_{k} \\ 0, & \text { if } t<t_{k}\end{cases}
$$


where $L_{k}=-\operatorname{div} A_{k} \nabla$. Observe that, for $t \in\left[t_{k}, t_{k+1}\right)$, and $s \in\left[t_{i}, t_{i+1}\right)$ with $i \leq k$, we have that

$$
\Gamma(t, s)=e^{-\left(t-t_{k}\right) L_{k}} e^{-\left(t_{k}-t_{k-1}\right) L_{k-1}} \ldots e^{-\left(t_{i+1}-s\right) L_{i}} .
$$

This was proven for finite sequences $\left(t_{j}\right)_{j=0, \ldots, N+1}$, but uniqueness in Theorem 3.11 gives us this formula even for infinite sequences. Thus we have that $v(t, \cdot)=w_{k}(t, \cdot)$ for all $t \in\left[t_{k}, t_{k+1}\right]$. Observe that for all $w \in L^{2}\left(\mathbb{R}^{n}\right)$ and $t \geq t_{k+1}$,

$$
\begin{aligned}
& e^{-\left(t-t_{k+1}\right) L_{k+1}}\left(e^{-\left(t_{k+1}-t_{k}\right) L_{k}} w\right) \\
& =e^{-\left(t-t_{k}\right) L_{k}} w-\int_{t_{k+1}}^{t} e^{-(t-\sigma) L_{k+1}} \operatorname{div}\left(A_{k+1}-A_{k}\right) \nabla e^{-\left(\sigma-t_{k}\right) L_{k}} w \mathrm{~d} \sigma .
\end{aligned}
$$

Hence, for $t \geq t_{k+1}$,

$$
w_{k+1}(t, \cdot)=w_{k}(t, \cdot)-\int_{t_{k+1}}^{t} e^{-(t-\sigma) L_{k+1}} \operatorname{div}\left(A_{k+1}-A_{k}\right) \nabla w_{k}(\sigma, \cdot) \mathrm{d} \sigma .
$$

Therefore, by Proposition 2.8 and the value of $p$, we have that

$$
\left\|1_{\left[t_{k+1}, \infty\right)} \nabla\left(w_{k+1}-w_{k}\right)\right\|_{T^{p, 2}} \leq\left\|\tilde{\mathcal{M}}_{L_{k+1}}\right\|_{\mathscr{L}\left(T^{p, 2}\right)}\left\|A_{k+1}-A_{k}\right\|_{L^{\infty}}\left\|1_{\left[t_{k+1}, \infty\right)} \nabla w_{k}\right\|_{T^{p, 2}} .
$$

The norms $\left\|\tilde{\mathcal{M}}_{L_{k+1}}\right\|_{\mathscr{L}\left(T^{p, 2}\right)}$ are uniformly bounded from our assumption $A_{k} \in \mathcal{M}(\Lambda, \lambda, q, M)$ for all $k \in \mathbb{N}$. Thus there exists a constant $C>0$, depending only on $p, q, M$ and the ellipticity constants in (1.2), such that

$$
\left\|\nabla w_{k+1}\right\|_{T^{p, 2}} \leq\left(1+C\left\|A_{k+1}-A_{k}\right\|_{L^{\infty}}\right)\left\|\nabla w_{k}\right\|_{T^{p, 2}} .
$$

Iterating, and using [9, Proposition 2.1], we have that

$$
\left\|\nabla w_{k}\right\|_{T^{p, 2}} \leq \prod_{j=0}^{k}\left(1+C\left\|A_{j+1}-A_{j}\right\|_{L^{\infty}}\right)\left\|\nabla w_{0}\right\|_{T^{p, 2}} \leq e^{C\|A\|_{B V\left(L^{\infty}\right)}}\left\|v_{0}\right\|_{L^{p}},
$$

since $\sum_{j=0}^{\infty}\left\|A_{j+1}-A_{j}\right\|_{L^{\infty}}=\|A\|_{B V\left(L^{\infty}\right)}$ for this particular $A$. So far, we have not used that $1<p<2$ in the statement. We note for further use that

$$
\sup _{k \in \mathbb{N}}\left\|1_{\left[t_{k}, t_{k+1}\right)} \nabla v\right\|_{T^{p, 2}} \leq e^{C\|A\|_{B V\left(L^{\infty}\right)}}\left\|v_{0}\right\|_{L^{p}} .
$$

The estimate on $w_{k}$ is sufficient to control $\|v(t, \cdot)\|_{L^{p}}$ when $1<p<2$ as we now show. Let $t \in\left[t_{k}, t_{k+1}\right)$ for some $k \in \mathbb{N}$. Using successively that $A_{k} \in \mathcal{M}(\Lambda, \lambda, q, M)$ for all $k \in \mathbb{N}$, [5, Corollary 3.6 and Theorem 5.1] , a change of variable $s \mapsto s-t_{k}$ in the fourth line and $p<2$ in the fifth line in applying [9, Proposition 2.1], we have the following chain of inequalities, with constants independent of $t$ and $k$ :

$$
\begin{aligned}
\|v(t, \cdot)\|_{L^{p}} & =\left\|e^{-\left(t-t_{k}\right) L_{k}} v_{k}\right\|_{L^{p}} \\
& \lesssim\left\|v_{k}\right\|_{L^{p}} \\
& \lesssim\left\|\left(\int_{0}^{\infty}\left|\nabla e^{-s L_{k}} v_{k}\right|^{2} \mathrm{~d} s\right)^{\frac{1}{2}}\right\|_{L^{p}} \\
& =\left\|\left(\int_{0}^{\infty}\left|\nabla w_{k}(s, .)\right|^{2} \mathrm{~d} s\right)^{\frac{1}{2}}\right\|_{L^{p}} \\
& \lesssim\left\|\nabla w_{k}\right\|_{T^{p, 2}} \leq e^{C\|A\|_{B V\left(L^{\infty}\right)}}\left\|v_{0}\right\|_{L^{p}}
\end{aligned}
$$


Theorem 6.9. Let $A \in L^{\infty}\left(\mathbb{R}_{+}^{n+1} ; \mathscr{M}_{n}(\mathbb{C})\right) \cap B V\left(L^{\infty}\right)$ satisfy (1.2) with constants $\Lambda, \lambda$. Let $q \in[1,2)$, and $M:\left[2, q^{\prime}\right) \rightarrow(0, \infty)$ be such that $A_{I} \in \mathcal{M}(\Lambda, \lambda, q, M)$ for all bounded intervals $I$ of $\mathbb{R}_{+}$.

Let $p \in\left(\max \left\{1, \frac{2 n}{n+q^{\prime}}\right\}, 2\right)^{1}$ and $u_{0} \in L^{p}\left(\mathbb{R}^{n}\right)$. Then

(i) $\sup _{0<s<t<\infty}\|\Gamma(t, s)\|_{\mathscr{L}\left(L^{p}\right)}<\infty$.

(ii) The function $u:(t, x) \mapsto \Gamma(t, 0) u_{0}(x)$ is the unique global weak solution of (1.1) in $L^{\infty}\left(L^{p}\right)$ or in $X^{p}$ such that $u(0, \cdot)=u_{0}$.

Moreover, $u \in \mathscr{C}_{0}\left(L^{p}\right)$, and $\|u\|_{L^{\infty}\left(L^{p}\right)} \sim\left\|u_{0}\right\|_{L^{p}} \sim\|u\|_{X^{p}}$.

(iii) The solution $u$ given in (ii) is such that $\nabla u \in T^{p, 2}$, and $\|\nabla u\|_{T^{p, 2}} \sim\left\|u_{0}\right\|_{L^{p}}$.

Proof. By Corollary 5.10, we have that (i) implies (ii).

Next, $($ ii $) \Longrightarrow$ (iii) is proven, using independent arguments that do not rely on the $B V\left(L^{\infty}\right)$ assumption, in Proposition 7.1 and Theorem 7.3.

Let us now prove $(i)$. Assume that $u_{0} \in L^{2}\left(\mathbb{R}^{n}\right) \cap L^{p}\left(\mathbb{R}^{n}\right)$. Let $\left\{A_{j}, j \in \mathbb{N}\right\}$ be the family of approximations of $A$ defined in Lemma 6.6. Let $u^{(j)}$ denote the corresponding global weak solution of $\partial_{t} v=\operatorname{div} A_{j} \nabla v$. By Lemma 6.7, we have that $\left\|u_{0}\right\|_{L^{p}} \sim\left\|u^{(j)}\right\|_{L^{\infty}\left(L^{p}\right)}$ for all $j \in \mathbb{N}$ with implied constants independent of $j$. Moreover $\left\|u_{0}\right\|_{L^{2}} \sim\left\|u^{(j)}\right\|_{L^{\infty}\left(L^{2}\right)} \sim\left\|\nabla u^{(j)}\right\|_{L^{2}\left(L^{2}\right)}$ uniformly in $j \in \mathbb{N}$. Therefore, there exists a subsequence $\left(v^{(j)}\right)_{j \in \mathbb{N}}$ of $\left(u^{(j)}\right)_{j \in \mathbb{N}}$, a function $v \in L^{\infty}\left(L^{2}\right)$, and a function $u \in L^{\infty}\left(L^{p}\right)$ such that

$$
\begin{array}{rll}
v^{(j)} & \underset{j \rightarrow \infty}{\longrightarrow} v & \text { weak }^{*} \text { in } L^{\infty}\left(L^{2}\right), \\
\nabla v^{(j)} & \stackrel{j \rightarrow \infty}{\longrightarrow} \nabla v & \text { weak }^{*} \text { in } L^{2}\left(L^{2}\right), \\
v^{(j)} & \stackrel{j \rightarrow \infty}{\longrightarrow} u & \text { weak }^{*} \text { in } L^{\infty}\left(L^{p}\right) .
\end{array}
$$

We have that $v=u$ as distributions, and that $v$ is a global weak solution of (1.1). By Theorem 3.11, it follows that $v(t, x)=\Gamma(t, 0) u_{0}(x)$ for all $t \geq 0$ and almost every $x \in \mathbb{R}^{n}$. Therefore, for all $t \geq 0,\left\|\Gamma(t, 0) u_{0}\right\|_{L^{p}}=\|v(t, .)\|_{L^{p}} \lesssim\left\|u_{0}\right\|_{L^{p}}$. Thus $\Gamma(t, 0)$ extends to a bounded operator on $L^{p}\left(\mathbb{R}^{n}\right)$, with norm independent of $t$. Starting at $s>0$ instead of 0 gives in the same way that $\sup _{t \in[s, \infty)}\|\Gamma(t, s)\|_{\mathscr{L}\left(L^{p}\right)}$ is controlled by the $B V\left(L^{\infty}\right)$ norm of $A$ on the interval $[s, \infty)$, which is smaller than the one on $[0, \infty)$.

Remark 6.10. Curiously, we are not able to prove (iii) using the approach of Lemma 6.7.

Remark 6.11. In the general situation, we can obtain all values of $p \in(1,2)$ if $n=1,2$, and all values $p \in\left(\frac{2 n}{n+2}-\varepsilon(\Lambda, \lambda), 2\right)$ if $n \geq 3$. If $A(t, x)$ depends only on $t$ or is periodic and continuous with respect to $x$ for all $t>0$ with common period, or even almost periodic for all $t>0$, then we obtain $p \in(1,2)$ in any dimension.

\subsection{Existence and uniqueness for $p<2$ : small perturbations of autonomous equations or continuous coefficients}

We now turn to an existence and uniqueness result for small perturbations of an autonomous problem or for continuous coefficients on a finite interval. We start with the following variant of Duhamel's formula.

\footnotetext{
${ }^{1}$ The range can be larger according to Remark 6.8 .
} 
Lemma 6.12. Let $f \in L^{2}\left(L^{2}\right)$ and $h \in L^{2}\left(\mathbb{R}^{n}\right)$. Let $\underline{A} \in L^{\infty}\left(\mathbb{R}^{n}, \mathscr{M}_{n}(\mathbb{C})\right)$ satisfy (1.2) and $L=-\operatorname{div} \underline{A} \nabla$. Define, for all $t>0$,

$$
u(t, \cdot)=e^{-t L} h+\mathcal{R}_{L} f(t, \cdot),
$$

where

$$
\mathcal{R}_{L} f:(t, x) \mapsto \int_{0}^{t} e^{-(t-s) L} \operatorname{div} f(s, \cdot)(x) \mathrm{d} s,
$$

is the bounded operator from $T^{2,2}$ to $X^{2}$ from Proposition 2.12. Then u is the unique element of $\dot{W}(0, \infty)$ such that, for all $\phi \in \mathscr{D}$,

$$
\left\langle u, \partial_{t} \phi\right\rangle=\langle\underline{A} \nabla u, \nabla \phi\rangle+\langle f, \nabla \phi\rangle,
$$

and $\operatorname{Tr}(u)=h$.

Proof. We first assume that $f \in \mathscr{D}$. Define $v_{0}:(t, x) \mapsto e^{-t L} h(x)$ and

$$
v=v_{0}+\mathcal{R}_{L} f .
$$

By semigroup theory, $v \in \mathscr{C}\left(L^{2}\right)$, and satisfies $\partial_{t} v=-L v_{0}+\operatorname{div} f$. By Proposition 2.13 and Step 0 of the proof of Theorem 3.11, we have that $\nabla v \in L^{2}\left(L^{2}\right)$, and thus

$$
\left\langle v, \partial_{t} \phi\right\rangle=\langle\underline{A} \nabla v, \nabla \phi\rangle+\langle f, \nabla \phi\rangle,
$$

for all $\phi \in \mathscr{D}$, as well as $\operatorname{Tr}(v)=h$.

Now, we turn to a general $f \in L^{2}\left(L^{2}\right)$, and let $\left(f_{k}\right)_{k \in \mathbb{N}}$ be a sequence of functions in $\mathscr{D}$ converging to $f$ in $L^{2}\left(L^{2}\right)$. Define, for all $k \in \mathbb{N}$,

$$
u_{k}=v_{0}+\mathcal{R}_{L} f_{k}, \quad \text { and } \quad u=v_{0}+\mathcal{R}_{L} f .
$$

Then $u_{k} \underset{k \rightarrow \infty}{\longrightarrow} u$ in $X^{2}$ and $\nabla u_{k} \underset{k \rightarrow \infty}{\longrightarrow} \nabla u$ in $L^{2}\left(L^{2}\right)$, using Propositions 2.12 and 2.8 . Therefore

$$
\left\langle u, \partial_{t} \phi\right\rangle=\langle\underline{A} \nabla u, \nabla \phi\rangle+\langle f, \nabla \phi\rangle,
$$

for all $\phi \in \mathscr{D}$. Since $\operatorname{Tr}\left(v_{k}\right)=h$ for all $k \in \mathbb{N}$, and $\operatorname{Tr}$ is continuous from $\dot{W}(0, \infty)$ to $L^{2}$ by Lemma 3.1, we also have that $\operatorname{Tr}(u)=h$.

We turn to uniqueness. Let $\tilde{u} \in \dot{W}(0, \infty)$ be another solution of

$$
\left\langle\tilde{u}, \partial_{t} \phi\right\rangle=\langle\underline{A} \nabla \tilde{u}, \nabla \phi\rangle+\langle f, \nabla \phi\rangle
$$

for all $\phi \in \mathscr{D}$, with $\operatorname{Tr}(\tilde{u})=h$. Then $w=u-\tilde{u}$ is a solution of

$$
\partial_{t} w=\operatorname{div} \underline{A} \nabla w, \quad w \in \dot{W}(0, \infty), \quad \operatorname{Tr}(w)=0,
$$

and thus $u=\tilde{u}$ by Theorem 3.11.

Corollary 6.13. Let $A \in L^{\infty}\left(\mathbb{R}_{+}^{n+1}, \mathscr{M}_{n}(\mathbb{C})\right)$ and $\underline{A} \in L^{\infty}\left(\mathbb{R}^{n}, \mathscr{M}_{n}(\mathbb{C})\right)$ satisfy (1.2). Let $L=-\operatorname{div} \underline{A} \nabla$. For all $t>0$ and $h \in L^{2}\left(\mathbb{R}^{n}\right)$, the following holds in $L^{2}\left(\mathbb{R}^{n}\right)$ :

$$
\Gamma(t, 0) h=e^{-t L} h+\int_{0}^{t} e^{-(t-s) L} \operatorname{div}(A(s, .)-\underline{A}) \nabla \Gamma(s, 0) h \mathrm{~d} s .
$$


Proof. Let $h \in L^{2}\left(\mathbb{R}^{n}\right)$, define $v_{0}(t, \cdot)=e^{-t L} h$, and $f(t, \cdot)=(A(t, \cdot)-\underline{A}) \nabla \Gamma(t, 0) h$ for all $t>0$. We have that $f \in L^{2}\left(L^{2}\right)$ by Theorem 3.11. Define $u=v_{0}+\mathcal{R}_{L} f$, and $\tilde{u}(t,)=.\Gamma(t, 0) h$ for all $t>0$. Using Lemma 6.12, we have the following, for all $\phi \in \mathscr{D}$ :

$$
\left\langle u, \partial_{t} \phi\right\rangle=\langle\underline{A} \nabla u, \nabla \phi\rangle+\langle(A-\underline{A}) \nabla \tilde{u}, \nabla \phi\rangle,
$$

Since $\tilde{u} \in \dot{W}(0, \infty)$ is a global weak solution of (1.1) with $\operatorname{Tr}(\tilde{u})=h$, we have that $u-\tilde{u} \in$ $\dot{W}(0, \infty)$ is a global weak solution of $\partial_{t}(u-\tilde{u})=\operatorname{div} \underline{A} \nabla(u-\tilde{u})$, with $\operatorname{Tr}(u-\tilde{u})=0$. Therefore $u=\tilde{u}$ by Theorem 3.11.

Theorem 6.14. Let $q \in[1,2)$, and $M:\left[2, q^{\prime}\right) \rightarrow(0, \infty)$, and let $\underline{A} \in \mathcal{M}(\Lambda, \lambda, q, M)$. Let $p \in\left(\max \left\{1, \frac{2 n}{n+q^{\prime}}\right\}, 2\right)$ and assume

$$
\varepsilon:=\|A-\underline{A}\|_{L^{\infty}}<\frac{1}{\left\|\tilde{\mathcal{M}}_{L}\right\|_{\mathcal{L}\left(T^{p, 2}\right)}}
$$

where $L=-\operatorname{div} \underline{A} \nabla$. Then,

$$
\sup _{0 \leq s \leq t<\infty}\|\Gamma(t, s)\|_{\mathscr{L}\left(L^{p}\right)}<\infty .
$$

Consequently, the conclusions of Corollary 5.10 hold in any open subinterval $(r, 2)$ on which (6.3) is valid.

Proof. Let $u_{0} \in L^{p}\left(\mathbb{R}^{n}\right) \cap L^{2}\left(\mathbb{R}^{n}\right)$, and define $u(t, \cdot)=\Gamma(t, 0) u_{0}$. We want to show that $\|u\|_{L^{\infty}\left(L^{p}\right)} \lesssim\left\|u_{0}\right\|_{L^{p}}$ with constant independent of $u_{0}$.

Let us first assume that $A$ is of the form

$$
A_{j}(t, x)=\sum_{m=0}^{4^{j}-1} \mathbb{1}_{\left[\frac{m}{2^{j}}, \frac{m+1}{2^{j}}\right)}(t) f_{\frac{m}{2^{j}}}^{\frac{m+1}{2^{j}}} A(s, x) \mathrm{d} s+\mathbb{1}_{\left[2^{j}, \infty\right)}(t) \underline{A}(x),
$$

for some $j \in \mathbb{N}$ and almost every $(t, x) \in(0, \infty) \times \mathbb{R}^{n}$. With this hypothesis, applying (6.1) $4^{j}+1$ times, we have the a priori information that $\nabla u \in T^{p, 2}$ with norm depending on $j$. However, we first show that $\|\nabla u\|_{T^{p, 2}} \sim\left\|u_{0}\right\|_{L^{p}}$ independently of $j$. Then we deduce a bound on $\|u\|_{X^{p}}$ and, finally, a bound on $\|u\|_{L^{\infty}\left(L^{p}\right)}$.

Step 1. Using the representation (6.2) with $v(t, \cdot)=e^{-t L} u_{0}$ for all $t>0$, we also have that

$$
\|\nabla u\|_{T^{p, 2}} \leq\|\nabla v\|_{T^{p, 2}}+\left\|\tilde{\mathcal{M}}_{L}(A-\underline{A}) \nabla u\right\|_{T^{p, 2}} .
$$

Using [5, Corollary 6.10] and Proposition 2.8 (Recall that $p>\frac{n q}{n+q}$ which is the exponent found in [5] and $p<2$ ), this gives us that

$$
\|\nabla u\|_{T^{p, 2}} \leq C\left\|u_{0}\right\|_{L^{p}}+\varepsilon\left\|\tilde{\mathcal{M}}_{L}\right\|_{\mathcal{L}\left(T^{p, 2}\right)}\|\nabla u\|_{T^{p, 2}},
$$

for some constant $C>0$. Therefore, with $C^{\prime}=C\left(1-\varepsilon\left\|\tilde{\mathcal{M}}_{L}\right\|_{\mathcal{L}\left(T^{p, 2}\right)}\right)^{-1}$, independent of $j$, we have $\|\nabla u\|_{T^{p, 2}} \leq C^{\prime}\left\|u_{0}\right\|_{L^{p}}$. Using (6.2) with $L=-\Delta$ and $w(t, \cdot)=e^{t \Delta} u_{0}$ for all $t>0$, together with a classical conical Littlewood-Paley estimate for $w$, we have that

$$
\left\|u_{0}\right\|_{L^{p}} \sim\|\nabla w\|_{T^{p, 2}} \lesssim\|\nabla u\|_{T^{p, 2}}+\left\|\tilde{\mathcal{M}}_{-\Delta}(A-I) \nabla u\right\|_{T^{p, 2}} \lesssim\|\nabla u\|_{T^{p, 2}}
$$


Step 2. Using Proposition 2.12 together with the representation (6.2) with $L=-\Delta$ and step 1 , we have that

$$
\|u\|_{X^{p}} \lesssim\|w\|_{X^{p}}+\|\nabla u\|_{T^{p, 2}} \lesssim\|w\|_{X^{p}}+\left\|u_{0}\right\|_{L^{p}} .
$$

The $L^{p}$ boundedness of the non-tangential maximal function for $w$ yields $\|w\|_{X^{p}} \lesssim\left\|u_{0}\right\|_{L^{p}}$, hence $\|u\|_{X^{p}} \lesssim\left\|u_{0}\right\|_{L^{p}}$.

Step 3. For $t>0$, using Hölder's inequalities as $p<2$ and Proposition 3.6, we have

$$
\|u(t, \cdot)\|_{L^{p}}^{p}=\int_{\mathbb{R}^{n}} f_{B(x, \sqrt{t})}|u(t, x)|^{p} \mathrm{~d} x \mathrm{~d} y \lesssim \int_{\mathbb{R}^{n}}\left(f_{B(y, \sqrt{t})}|u(t, x)|^{2} \mathrm{~d} x\right)^{\frac{p}{2}} \mathrm{~d} y \lesssim\|u\|_{X^{p}}^{p} .
$$

For all $u_{0} \in L^{p}\left(\mathbb{R}^{n}\right) \cap L^{2}\left(\mathbb{R}^{n}\right)$, we thus have obtained from this and step 2 that

$$
\sup _{t>0}\|u(t, \cdot)\|_{L^{p}} \lesssim\left\|u_{0}\right\|_{L^{p}}
$$

The operators $\Gamma(t, 0)$ thus extend to bounded operators on $L^{p}\left(\mathbb{R}^{n}\right)$, and one has the uniform estimate $\sup _{0<t}\|\Gamma(t, 0)\|_{\mathscr{L}\left(L^{p}\right)}<\infty$. Furthermore, we obtain strong continuity of $\Gamma(t, 0)$ at 0 in $L^{p}\left(\mathbb{R}^{n}\right)$ from the one on $L^{2}\left(\mathbb{R}^{n}\right)$ as we work for $p$ in an open interval. Thus, $\left\|u_{0}\right\|_{L^{p}} \leq$ $\|u\|_{L^{\infty}\left(L^{p}\right)}$. In conclusion, we have shown that

$$
\|u\|_{X^{p}} \sim\left\|u_{0}\right\|_{L^{p}} \sim\|\nabla u\|_{T^{p, 2}} \sim\|u\|_{L^{\infty}\left(L^{p}\right)} .
$$

The same reasoning gives us that $\sup _{0 \leq s \leq t<\infty}\|\Gamma(t, s)\|_{\mathscr{L}\left(L^{p}\right)}<\infty$. Note that the bound is uniform for all $j$.

The rest of the proof is identical to the proof of Theorem 6.9, using the family $\left\{A_{j}, j \in \mathbb{N}\right\}$ of approximations of $A$ at the beginning of the proof rather than the approximations given by Lemma 6.6.

Theorem 6.15. Assume $A \in \mathscr{C}\left([0, T] ; L^{\infty}\left(\mathbb{R}^{n} ; \mathscr{M}_{n}(\mathbb{C})\right)\right)$ and that there are $q \in[1,2)$, and $M:\left[2, q^{\prime}\right) \rightarrow(0, \infty)$ such that $A(s, \cdot) \in \mathcal{M}(\Lambda, \lambda, q, M)$ for all $s \in[0, T]$. For $p \in$ $\left(\max \left\{1, \frac{2 n}{n+q^{\prime}}\right\}, 2\right)$, we have that

$$
\sup _{0 \leq s \leq t<\leq T}\|\Gamma(t, s)\|_{\mathcal{L}\left(L^{p}\right)}<\infty .
$$

Consequently, the conclusions of Corollary 5.10 hold replacing $t>0$ by $t \in(0, T]$, global solutions by local solutions on $(0, T)$, and $\mathscr{C}_{0}\left(L^{p}\right)$ by $\mathscr{C}\left([0, T] ; L^{p}\right)$.

Proof. Let $u_{0} \in L^{p}\left(\mathbb{R}^{n}\right) \cap L^{2}\left(\mathbb{R}^{n}\right)$ and define $u(t, \cdot)=\Gamma(t, 0) u_{0}$. We want to show (6.4). To do so, we adapt the proof of Theorem 6.14. For $\varepsilon>0$, choose $\delta>0$ according to the uniform continuity of $A$ on $[0, T]$ such that $\left\|A(s, \cdot)-A\left(s^{\prime}, \cdot\right)\right\|_{L^{\infty}}<\varepsilon$ if $\left|s-s^{\prime}\right| \leq 2 \delta$. We may assume that $\delta=\frac{T}{2^{k}}$ for some $k \in \mathbb{N}$.

We begin by replacing $A$ by $A_{j}(t, x)=\sum_{m=0}^{2^{j}-1} 1_{\left[\frac{m T}{2^{j}}, \frac{(m+1) T}{2^{j}}\right)}(t) A\left(\frac{m T}{2^{j}}, x\right)$, for $j>k$. We still denote the solution by $u$ to keep the notation simple. With such coefficients, we know from (6.1) that $\|\nabla u\|_{T^{p, 2}}<\infty$ qualitatively.

Step 1. Using the representation (6.2) with $v(, \cdot)=e^{-t L} u_{0}, L=-\operatorname{div} A(0, \cdot) \nabla$, we have

$$
\left.\left\|1_{(0,2 \delta)} \nabla u\right\|_{T^{p, 2}} \leq\left\|1_{(0,2 \delta)} \nabla v\right\|_{T^{p, 2}}+\| 1_{(0,2 \delta)} \tilde{\mathcal{M}}_{L}\left(A_{j}-A(0, \cdot)\right) \nabla u\right) \|_{T^{p, 2}} .
$$


Note that the truncation implies that the only values of $A(s, x)$ that play a role are those for $s \in[0,2 \delta]$. Thus as $j \geq k$, we obtain,

$$
\left\|1_{(0,2 \delta)} \nabla u\right\|_{T^{p, 2}} \leq C\left\|u_{0}\right\|_{L^{p}}+\varepsilon\left\|\tilde{\mathcal{M}}_{L}\right\|_{\mathscr{L}\left(T^{p, 2}\right)}\left\|1_{(0,2 \delta)} \nabla u\right\|_{T^{p, 2}},
$$

for some constant $C>0$ independent of $j$. Therefore, having first chosen $\varepsilon>0$ with $\varepsilon\left\|\mathcal{M}_{L}\right\|_{\mathscr{L}\left(T^{p, 2}\right)}<1$, using the finiteness of $\left\|1_{(0,2 \delta)} \nabla u\right\|_{T^{p, 2}}$, we have $\left\|1_{(0,2 \delta)} \nabla u\right\|_{T^{p, 2}} \leq$ $C^{\prime}\left\|u_{0}\right\|_{L^{p}}$, with $C^{\prime}=C\left(1-\varepsilon\left\|\tilde{\mathcal{M}}_{L}\right\|_{\mathscr{L}\left(T^{p, 2}\right)}\right)^{-1}$.

Step 2. Set $w(t, \cdot)=e^{t \Delta} u_{0}$. Using Proposition 2.12 together with the representation (6.2) with $L=-\Delta$ and step 1 , we have that

$$
\left\|1_{(0,2 \delta)} u\right\|_{X^{p}} \lesssim\left\|\mathbb{1}_{(0,2 \delta)} w\right\|_{X^{p}}+\left\|\mathbb{1}_{(0,2 \delta)} \nabla u\right\|_{T^{p, 2}} \lesssim\|w\|_{X^{p}}+\left\|u_{0}\right\|_{L^{p}}
$$

The $L^{p}$ boundedness of the non-tangential maximal function for $w$ yields $\|w\|_{X^{p}} \lesssim\left\|u_{0}\right\|_{L^{p}}$, hence $\left\|1_{(0,2 \delta)} u\right\|_{X^{p}} \lesssim\left\|u_{0}\right\|_{L^{p}}$.

Step 3. For $0<t \leq \delta$, using Hölder's inequalities as $p<2$ and Proposition 3.6, we have

$$
\|u(t, \cdot)\|_{L^{p}}^{p}=\int_{\mathbb{R}^{n}} f_{B(x, \sqrt{t})}|u(t, x)|^{p} \mathrm{~d} x \mathrm{~d} y \lesssim \int_{\mathbb{R}^{n}}\left(f_{B(y, \sqrt{t})}|u(t, x)|^{2} \mathrm{~d} x\right)^{\frac{p}{2}} \mathrm{~d} y \lesssim\left\|1_{(0,2 \delta)} u\right\|_{X^{p}}^{p} .
$$

For all $u_{0} \in L^{p}\left(\mathbb{R}^{n}\right) \cap L^{2}\left(\mathbb{R}^{n}\right)$, we thus have that

$$
\sup _{0<t \leq \delta}\|u(t, \cdot)\|_{L^{p}} \lesssim\left\|u_{0}\right\|_{L^{p}}
$$

Therefore, the operators $\Gamma(t, 0)$ extend to bounded operators on $L^{p}\left(\mathbb{R}^{n}\right)$, and one has the uniform estimate $\sup _{0<t<\delta}\|\Gamma(t, 0)\|_{\mathscr{L}\left(L^{p}\right)}<\infty$. Furthermore, we obtain strong continuity of $\Gamma(t, 0)$ at 0 in $L^{p}\left(\mathbb{R}^{n}\right)$ from the one on $L^{2}\left(\mathbb{R}^{n}\right)$ as we work for $p$ in an open interval. In conclusion, we have shown that

$$
\left\|u_{0}\right\|_{L^{p}} \sim \sup _{0 \leq t \leq \delta}\|u(t, \cdot)\|_{L^{p}} .
$$

Given the form of $A_{j}$, one can obtain similarly $\sup _{m \delta \leq s \leq t \leq(m+1) \delta}\|\Gamma(t, s)\|_{\mathscr{L}\left(L^{p}\right)}<\infty$. Iterating at most $\frac{T}{\delta}$ times, using the reproducing formula for the propagators, we obtain $\sup _{0 \leq s \leq t \leq T}\|\Gamma(t, s)\|_{\mathscr{L}\left(L^{p}\right)}<\infty$.

We conclude for $A$ as in Theorem 6.9 using the above approximations $A_{j}$ of $A$, remarking that the bound obtained for the propagators of $A_{j}$ are uniform for $j$ large enough and depend solely on the uniform continuity assumption and $T$.

Remark 6.16. In this argument, we only used properties of the semigroups for each coefficients $A(s, \cdot)$ on a bounded interval. Thus we may replace the assumption $A(s, \cdot) \in$ $\mathcal{M}(\Lambda, \lambda, q, M)$ for all $s \in[0, T]$, by $A(s, \cdot) \in \mathcal{M}_{T}(\Lambda, \lambda, q, M)$ for all $s \in[0, T]$. The subscript $T$ means that we consider the supremum in Definition 6.1 taken on $(0, T]$. For example, any $A \in L^{\infty}\left(\mathbb{R}^{n}, \mathscr{M}_{n}(\mathbb{C})\right.$ ) that is uniformly continuous (or even that belongs to $V M O$ ) on $\mathbb{R}^{n}$ belongs to $\mathcal{M}_{T}(\Lambda, \lambda, 1, M)$ for some $M$ and all $T>0$; see [4]. In particular for any $A \in \mathscr{C}\left([0, \infty) ; L^{\infty}\left(\mathbb{R}^{n} ; \mathscr{M}_{n}(\mathbb{C})\right)\right)$ such that $A(s, \cdot)$ is uniformly continuous on $\mathbb{R}^{n}$, uniformly for $s \geq 0$ (the uniformity in $s$ is imposed to guarantee that we have the same function $M$ for all $A(s, \cdot))$, we can apply our result with $p \in(1,2)$ and obtain global solutions in $\mathscr{C}\left(L^{p}\right)$ (but not bounded). For example, it applies to any $A$ which is uniformly continuous on $\overline{\mathbb{R}_{+}^{n+1}}$. 


\section{Square functions and maximal functions a priori estimates}

We prove here some comparisons between conical square functions in $L^{p}\left(\mathbb{R}^{n}\right)$, namely $\|\nabla u\|_{T^{p, 2}}$ and non-tangential maximal functions in $L^{p}$, namely $\|u\|_{X^{p}}$ for weak solutions of $\partial_{t} u=\operatorname{div} A \nabla u$. In the case of autonomous equations, such bounds are obtained in [22] for $p \geq 1$. It is tempting to study the cases where $p<1$ as well, but this is outside the scope of the present work.

\subsection{Controlling the maximal function by the square function for $1 \leq p<\infty$}

As a consequence of Proposition 2.12 and classical Littlewood-Paley theory, we first obtain the following control of the maximal function by the square function.

Proposition 7.1. Let $1 \leq p<\infty, u_{0} \in L^{2}\left(\mathbb{R}^{n}\right)$, and $u(t, \cdot)=\Gamma(t, 0) u_{0}$ for all $t>0$. If $\nabla u \in T^{p, 2}$, then $u \in X^{p}$, and

$$
\|u\|_{X^{p}} \lesssim\|\nabla u\|_{T^{p, 2}}
$$

with implicit constant independent of $u$.

Proof. Set $v(t,)=.e^{t \Delta} u_{0}$ for all $t>0$. Using (6.2) with $L=-\Delta$ and Proposition 2.12, we have that

$$
\|u\|_{X^{p}} \lesssim\|v\|_{X^{p}}+\left\|\mathcal{R}_{-\Delta}\right\|_{\mathcal{L}\left(T^{p, 2}, X^{p}\right)}\|A-I\|_{L^{\infty}}\|\nabla u\|_{T^{p, 2}} .
$$

Using (6.2) again, together with the classical conical Littlewood-Paley estimate, and Proposition 2.8 , we also have that

$$
\|v\|_{X^{p}} \lesssim\left\|v^{*}\right\|_{L^{p}} \lesssim\|\nabla v\|_{T^{p, 2}} \lesssim\|\nabla u\|_{T^{p, 2}}+\left\|\tilde{\mathcal{M}}_{-\Delta}(A-I) \nabla u\right\|_{T^{p, 2}} \lesssim\|\nabla u\|_{T^{p, 2}} .
$$

Note that the range can be improved to $p>\frac{n}{n+1}$, which is the same range as for the classical conical Littlewood-Paley estimate.

As a corollary, we have the following improvement of Theorem 3.11.

Corollary 7.2. For all $u_{0} \in L^{2}\left(\mathbb{R}^{n}\right)$, the problem

$$
\partial_{t} u=\operatorname{div} A \nabla u, \quad u \in X^{2}, \quad \operatorname{Tr}(u)=u_{0}
$$

is well-posed. Moreover, the solution $u$ is the energy solution, i.e. $u(t, \cdot)=\Gamma(t, 0) u_{0}$ for all $t>0$, and

$$
\left\|u_{0}\right\|_{L^{2}}=\|u\|_{L^{\infty}\left(L^{2}\right)} \lesssim\|u\|_{X^{2}} \lesssim\|\nabla u\|_{L^{2}\left(L^{2}\right)} \leq \sqrt{\frac{1}{2 \lambda}}\left\|u_{0}\right\|_{L^{2}} .
$$

Proof. Existence. Let $u(t, \cdot)=\Gamma(t, 0) u_{0}$ for all $t>0$. By Proposition 7.1 and Theorem 3.11, we have that

$$
\|u\|_{X^{2}} \lesssim\|\nabla u\|_{T^{2,2}}=\|\nabla u\|_{L^{2}\left(L^{2}\right)} \leq \sqrt{\frac{1}{2 \lambda}}\left\|u_{0}\right\|_{L^{2}} .
$$

Uniqueness. We have by Proposition 3.6 that $\|u\|_{L^{\infty}\left(L^{2}\right)} \lesssim\|u\|_{X^{2}}$ for all $u \in X^{2}$, and $L^{\infty}\left(L^{2}\right)$ is a class of uniqueness as shown in Theorem 5.2. 


\subsection{Controlling the square function by the maximal function for $p \in[1,2)$}

Theorem 7.3. Let $u$ be a global weak solution of (1.1). Let $p \in[1,2)$, and assume that $u \in X^{p}$. Then $\nabla u \in T^{p, 2}$ and

$$
\|\nabla u\|_{T^{p, 2}} \lesssim\|u\|_{X^{p}}
$$

with constant depending only on the ellipticity parameters in (1.2).

Note that the proof works for $0<p<1$ as well with the definitions of $T^{p, 2}$ and $X^{p}$ extended to these values.

Proof. The proof is highly similar to its autonomous counterpart in [22, Theorem 6.1], itself based on Fefferman-Stein's original argument for $L=-\Delta$ in [20]. The only difference is that we need to use cut-off functions rather than integration by parts to localise near the boundary of truncated cones, Proposition 3.6 instead of Caccioppoli's inequalities, and Kenig-Pipher's maximal function instead of the maximal function used in [22]. We include the full proof for the convenience of the reader. Let $\varepsilon, R, \sigma>0$ with $\varepsilon<\frac{R}{6}$. Pick $\beta>0$ to be determined later. Recall that

$$
\mathcal{N}_{\beta}(u)(x)=\sup _{\delta>0}\left(f_{\delta^{2}}^{\beta^{2} \delta^{2}} f_{B(x, \beta \delta)}|u(t, y)|^{2} \mathrm{~d} y \mathrm{~d} t\right)^{\frac{1}{2}} \quad \forall x \in \mathbb{R}^{n} .
$$

Define $E=\left\{x \in \mathbb{R}^{n} ; \mathcal{N}_{\beta} u(x) \leq \sigma\right\}$, and $E^{*}=\{x \in E ; \forall r>0 \quad|B(x, r) \cap E| \geq$ $\left.\frac{1}{2}|B(x, r)|\right\}$. Let $B=E^{c}, B^{*}=\left(E^{*}\right)^{c}$, and, for $x \in \mathbb{R}^{n}, \alpha>0$,

$$
\Gamma^{\varepsilon, R, \alpha}(x)=\left\{(t, y) \in[0, \infty) \times \mathbb{R}^{n} ;|y-x|<\alpha t \text { and } \varepsilon<t<R\right\},
$$

as well as $\mathcal{R}^{\varepsilon, R, \alpha}\left(E^{*}\right)=\bigcup_{x \in E^{*}} \Gamma^{\varepsilon, R, \alpha}(x)$.

We also define $\tilde{\mathcal{B}}^{\varepsilon, R}\left(E^{*}\right)=\tilde{\mathcal{B}}_{\varepsilon}\left(E^{*}\right) \cup \tilde{\mathcal{B}}^{R}\left(E^{*}\right) \cup \tilde{\mathcal{B}}^{\prime}\left(E^{*}\right)$, where

$$
\begin{aligned}
\tilde{\mathcal{B}}_{\varepsilon}\left(E^{*}\right) & =\left\{(t, y) \in[0, \infty) \times \mathbb{R}^{n} ; t \in(\varepsilon, 2 \varepsilon) \text { and } d\left(y, E^{*}\right)<t\right\}, \\
\tilde{\mathcal{B}}^{R}\left(E^{*}\right) & =\left\{(t, y) \in[0, \infty) \times \mathbb{R}^{n} ; t \in(R, 2 R) \text { and } d\left(y, E^{*}\right)<t\right\}, \\
\tilde{\mathcal{B}}^{\prime}\left(E^{*}\right) & =\left\{(t, y) \in[0, \infty) \times \mathbb{R}^{n} ; t \in(\varepsilon, 2 R) \text { and } t / 2 \leq d\left(y, E^{*}\right)<t\right\} .
\end{aligned}
$$

Note that

$$
\mathcal{R}^{\varepsilon, 2 R, 1}\left(E^{*}\right)=\mathcal{R}^{2 \varepsilon, R, 1 / 2}\left(E^{*}\right) \cup \tilde{\mathcal{B}}^{\varepsilon, R}\left(E^{*}\right) .
$$

We remark that

$$
\int_{E^{*}}\left(\int_{0}^{\infty} f_{B\left(x, \frac{\sqrt{t}}{2}\right)}|\nabla u(t, y)|^{2} \mathrm{~d} y \mathrm{~d} t\right) \mathrm{d} x=2 \int_{E^{*}}\left(\int_{0}^{\infty} f_{B\left(x, \frac{s}{2}\right)} s\left|\nabla u\left(s^{2}, y\right)\right|^{2} \mathrm{~d} y \mathrm{~d} s\right) \mathrm{d} x .
$$

and

$$
\int_{E^{*}}\left(\int_{2 \varepsilon}^{R} f_{B(x, s)} s\left|\nabla u\left(s^{2}, y\right)\right|^{2} \mathrm{~d} y \mathrm{~d} s\right) \mathrm{d} x \leq c_{n} \int_{\mathcal{R}^{2 \varepsilon, R, 1 / 2}\left(E^{*}\right)} s\left|\nabla u\left(s^{2}, y\right)\right|^{2} \mathrm{~d} y \mathrm{~d} s .
$$

with $c_{n}$ the reciprocal of the volume of the unit ball. We estimate the last integral. To do so, set

$$
\chi(t, y)=\left(1-\eta\left(\frac{2 d\left(y, E^{*}\right)}{t}\right)\right) \eta\left(\frac{t}{\varepsilon}\right)\left(1-\eta\left(\frac{t}{R}\right)\right) \quad \forall(t, y) \in \mathbb{R}_{+}^{n+1},
$$

where $\eta \in \mathscr{C}_{c}^{\infty}([0, \infty),[0,1])$ is equal to 1 on $[2, \infty)$, and equal to 0 on $[0,1]$. Notice that $\chi$ is almost everywhere differentiable, supported in $\mathcal{R}^{\varepsilon, 2 R, 1}\left(E^{*}\right)$, and constantly equal to 1 
on $\mathcal{R}^{2 \varepsilon, R, \frac{1}{2}}\left(E^{*}\right)$. Moreover, for all $(t, y) \in \mathcal{R}^{\varepsilon, 2 R, 1}\left(E^{*}\right)$, we have that $|\nabla \chi(t, y)| \leq \frac{2\left\|\eta^{\prime}\right\|_{\infty} \sqrt{n}}{t}$. For $(t, y) \in \tilde{\mathcal{B}}_{\varepsilon}\left(E^{*}\right)$, we have,

$$
\left|\partial_{t} \chi(t, y)\right| \leq 2\left\|\eta^{\prime}\right\|_{\infty}\left(\frac{1}{t}+\frac{d\left(y, E^{*}\right)}{t^{2}}\right) \leq \frac{4\left\|\eta^{\prime}\right\|_{\infty}}{t} .
$$

The same reasoning gives us that $\left|\partial_{t} \chi(t, y)\right| \leq \frac{4\left\|\eta^{\prime}\right\|_{\infty}}{t}$, for all $(t, y) \in \tilde{\mathcal{B}}^{R}\left(E^{*}\right)$. For $(t, y) \in$ $\tilde{\mathcal{B}}^{\prime}\left(E^{*}\right)$, we also have that $\left|\partial_{t} \chi(t, y)\right| \leq \frac{2\left\|\eta^{\prime}\right\|_{\infty} d\left(y, E^{*}\right)}{t^{2}} \leq \frac{2\left\|\eta^{\prime}\right\|_{\infty}}{t}$. Putting all these estimates together, we have shown that there exists $C>0$ such that for all $(t, y) \in \mathcal{R}^{\varepsilon, 2 R, 1}\left(E^{*}\right)$, we have that

$$
|\nabla \chi(t, y)|+\left|\partial_{t} \chi(t, y)\right| \leq \frac{C}{t}
$$

According to Remark 3.7, provided we show that $(s, x) \mapsto u\left(s^{2}, x\right) \chi^{2}(s, x) \in L^{2}\left(\varepsilon, 2 R ; H^{1}\left(\mathbb{R}^{n}\right)\right)$, we can use this function as a test function. We assume this for now, and estimate as follows:

$$
\begin{aligned}
\int_{\mathcal{R}^{2 \varepsilon, R, 1 / 2}\left(E^{*}\right)} s\left|\nabla u\left(s^{2}, y\right)\right|^{2} \mathrm{~d} y \mathrm{~d} s & \leq 2 \lambda \Re e \int_{\mathbb{R}_{+}^{n+1}} s \chi^{2}(s, y) A\left(s^{2}, y\right) \nabla u\left(s^{2}, y\right) \cdot \overline{\nabla u\left(s^{2}, y\right)} \mathrm{d} y \mathrm{~d} s \\
& \lesssim J_{1}+J_{2}+J_{3},
\end{aligned}
$$

where

$$
\begin{aligned}
& J_{1}=\left|\int_{\mathbb{R}_{+}^{n+1}} s A\left(s^{2}, y\right) \nabla u\left(s^{2}, y\right) \cdot \overline{u\left(s^{2}, y\right) \nabla\left(\chi^{2}(s, y)\right)} \mathrm{d} y \mathrm{~d} s\right|, \\
& J_{2}=\left|\int_{\mathbb{R}_{+}}\left\langle\partial_{s}\left(\chi(s, y) u\left(s^{2}, y\right)\right), u\left(s^{2}, y\right) \chi(s, y)\right\rangle \mathrm{d} s\right|, \\
& J_{3}=\left|\int_{\mathbb{R}_{+}^{n+1}} u\left(s^{2}, y\right) \partial_{s}(\chi(s, y)) \overline{u\left(s^{2}, y\right) \chi(s, y)} \mathrm{d} y \mathrm{~d} s\right| .
\end{aligned}
$$

Here, we notice that for each $s$, the bracket is the duality $H^{-1}\left(\mathbb{R}^{n}\right), H^{1}\left(\mathbb{R}^{n}\right)$. Hence, $J_{2}=$ $\frac{1}{2} \int_{\mathbb{R}_{+}} \partial_{s}\left\|\chi(s, \cdot) u\left(s^{2}, \cdot\right)\right\|_{L^{2}}^{2} \mathrm{~d} s=0$. Moreover,

$$
\begin{aligned}
J_{1} & \lesssim \int_{\tilde{\mathcal{B}} \varepsilon, R\left(E^{*}\right)}\left|u\left(s^{2}, y\right)\right|\left|\nabla u\left(s^{2}, y\right)\right| \mathrm{d} y \mathrm{~d} s, \\
J_{3} & \lesssim \int_{\tilde{\mathcal{B}}^{\varepsilon, R}\left(E^{*}\right)}\left|u\left(s^{2}, y\right)\right|^{2} \frac{\mathrm{d} y \mathrm{~d} s}{s} .
\end{aligned}
$$

This yields

$$
\begin{aligned}
& \int_{E^{*}}\left(\int_{2 \varepsilon}^{R} f_{B\left(x, \frac{s}{2}\right)} s\left|\nabla u\left(s^{2}, y\right)\right|^{2} \mathrm{~d} y \mathrm{~d} s\right) \mathrm{d} x \\
\lesssim & \int_{\tilde{\mathcal{B}}^{\varepsilon}, R\left(E^{*}\right)}\left|u\left(s^{2}, y\right)\right|^{2} \frac{\mathrm{d} y \mathrm{~d} s}{s}+\left(\int_{\tilde{\mathcal{B}}^{\varepsilon}, R\left(E^{*}\right)}\left|u\left(s^{2}, y\right)\right|^{2} \frac{\mathrm{d} y \mathrm{~d} s}{s}\right)^{\frac{1}{2}}\left(\int_{\tilde{\mathcal{B}}_{\varepsilon}, R\left(E^{*}\right)} s\left|\nabla u\left(s^{2}, y\right)\right|^{2} \mathrm{~d} y \mathrm{~d} s\right)^{\frac{1}{2}} .
\end{aligned}
$$

We now consider the following six integrals, recalling that $\tilde{\mathcal{B}}^{\varepsilon, R}\left(E^{*}\right)=\tilde{\mathcal{B}}_{\varepsilon}\left(E^{*}\right) \cup \tilde{\mathcal{B}}^{R}\left(E^{*}\right) \cup$ $\tilde{\mathcal{B}}^{\prime}\left(E^{*}\right)$.

$$
\begin{array}{rlrl}
I_{1} & =\int_{\tilde{\mathcal{B}}_{\varepsilon}\left(E^{*}\right)}\left|u\left(s^{2}, y\right)\right|^{2} \frac{\mathrm{d} y \mathrm{~d} s}{s}, & I_{2}=\int_{\tilde{\mathcal{B}}_{\varepsilon}\left(E^{*}\right)} s\left|\nabla u\left(s^{2}, y\right)\right|^{2} \mathrm{~d} y \mathrm{~d} s, \\
I_{3}=\int_{\tilde{\mathcal{B}}^{R}\left(E^{*}\right)}\left|u\left(s^{2}, y\right)\right|^{2} \frac{\mathrm{d} y \mathrm{~d} s}{s}, & I_{4}=\int_{\tilde{\mathcal{B}}^{R}\left(E^{*}\right)} s\left|\nabla u\left(s^{2}, y\right)\right|^{2} \mathrm{~d} y \mathrm{~d} s, \\
I_{5}=\int_{\tilde{\mathcal{B}}^{\prime}\left(E^{*}\right)}\left|u\left(s^{2}, y\right)\right|^{2} \frac{\mathrm{d} y \mathrm{~d} s}{s}, & I_{6}=\int_{\tilde{\mathcal{B}}^{\prime}\left(E^{*}\right)} s\left|\nabla u\left(s^{2}, y\right)\right|^{2} \mathrm{~d} y \mathrm{~d} s .
\end{array}
$$


For $I_{1}$, we have the following:

$$
\begin{aligned}
I_{1} & \lesssim \int_{\tilde{\mathcal{B}}_{\varepsilon}\left(E^{*}\right)}\left(\int_{E \cap B(y, s)} s^{-n} \mathrm{~d} x\right)\left|u\left(s^{2}, y\right)\right|^{2} \frac{\mathrm{d} y \mathrm{~d} s}{s} \\
& \lesssim \int_{\varepsilon}^{2 \varepsilon} \int_{E} f_{B(x, s)}\left|u\left(s^{2}, y\right)\right|^{2} \frac{\mathrm{d} y \mathrm{~d} s \mathrm{~d} x}{s} \lesssim \int_{E} \int_{\varepsilon^{2}}^{4 \varepsilon^{2}} f_{B(x, 2 \varepsilon)}|u(s, y)|^{2} \frac{\mathrm{d} y \mathrm{~d} s \mathrm{~d} x}{s} \\
& \lesssim \int_{E} \sup _{\delta>0} f_{\delta^{2}}^{4 \delta^{2}} f_{B(x, 2 \delta)}|u(s, y)|^{2} \mathrm{~d} y \mathrm{~d} s \mathrm{~d} x=\int_{E}\left|\mathcal{N}_{2} u(x)\right|^{2} \mathrm{~d} x .
\end{aligned}
$$

To handle $I_{2}$, we use Proposition 3.6, and a covering argument, to obtain the following:

$$
\begin{aligned}
I_{2} & \lesssim \int_{\varepsilon}^{2 \varepsilon} \int_{E} f_{B(x, 2 \varepsilon)} s\left|\nabla u\left(s^{2}, y\right)\right|^{2} \mathrm{~d} y \mathrm{~d} s \mathrm{~d} x \lesssim \int_{E} \int_{\varepsilon^{2}}^{4 \varepsilon^{2}} f_{B(x, 2 \varepsilon)}|\nabla u(s, y)|^{2} \mathrm{~d} y \mathrm{~d} s \mathrm{~d} x \\
& \lesssim \int_{E} f_{\varepsilon^{2} / 2}^{4 \varepsilon^{2}} f_{B(x, 4 \varepsilon)}|u(s, y)|^{2} \mathrm{~d} y \mathrm{~d} s \mathrm{~d} x=\int_{E}\left|\mathcal{N}_{4} u(x)\right|^{2} \mathrm{~d} x .
\end{aligned}
$$

In the same way $I_{3}+I_{4} \lesssim \int_{E}\left|\mathcal{N}_{4} u(x)\right|^{2} \mathrm{~d} x$. We now turn to $I_{5}, I_{6}$, using a Whitney decomposition of $B^{*}$ : there exist $c_{1}, c_{2} \in(0,1)$ with $c_{2}>c_{1}$, and $c_{3} \in \mathbb{N}$, such that $B^{*}=\bigcup_{k=0}^{\infty} B\left(x_{k}, r_{k}\right)$ for some $x_{k} \in \mathbb{R}^{n}$ and $r_{k}>0$ such that

$$
\begin{aligned}
& \forall k \in \mathbb{N} \quad c_{1} d\left(x_{k}, E^{*}\right) \leq r_{k} \leq c_{2} d\left(x_{k}, E^{*}\right), \\
& \forall x \in B^{*} \quad\left|\left\{k \in \mathbb{N} ; x \in B\left(x_{k}, r_{k}\right)\right\}\right| \leq c_{3} .
\end{aligned}
$$

We have the following:

$$
\begin{aligned}
I_{5} & \lesssim \sum_{k=0}^{\infty} \int_{r_{k}\left(\frac{1}{c_{2}}-1\right)}^{2 r_{k}\left(\frac{1}{c_{1}}+1\right)} \int_{B\left(x_{k}, r_{k}\right)}\left|u\left(s^{2}, y\right)\right|^{2} \frac{\mathrm{d} y \mathrm{~d} s}{s} \\
& \lesssim \sum_{k=0}^{\infty} r_{k}^{n} f_{r_{k}^{2}\left(\frac{1}{c_{2}}-1\right)^{2}}^{4 r_{k}^{2}\left(\frac{1}{c_{1}}+1\right)^{2}} f_{B\left(x_{k}, \frac{c_{2}}{1-c_{2}} \sqrt{s}\right)}|u(s, y)|^{2} \mathrm{~d} y \mathrm{~d} s .
\end{aligned}
$$

Now remark that, for $k \in \mathbb{N}$ and $s \in\left[r_{k}^{2}\left(\frac{1}{c_{2}}-1\right)^{2}, 4 r_{k}^{2}\left(\frac{1}{c_{1}}+1\right)^{2}\right]$ :

$$
d\left(x_{k}, E\right) \leq d\left(x_{k}, E^{*}\right) \leq \frac{r_{k}}{c_{1}} \leq \frac{c_{2}}{c_{1}\left(1-c_{2}\right)} \sqrt{s} .
$$

Therefore, there exists $x_{k}^{\prime} \in E$ such that

$$
B\left(x_{k}, \frac{c_{2}}{1-c_{2}} \sqrt{s}\right) \subset B\left(x_{k}^{\prime}, \frac{c_{2}}{1-c_{2}}\left(\frac{1}{c_{1}}+1\right) \sqrt{s}\right) .
$$

This yields

$$
I_{5} \lesssim \sum_{k=0}^{\infty} r_{k}^{n} \sup _{k \in \mathbb{N}}\left(f_{r_{k}^{2}\left(\frac{1}{c_{2}}-1\right)^{2}}^{4 r_{k}^{2}\left(\frac{1}{c_{1}}+1\right)^{2}} f_{B\left(x_{k}^{\prime}, \frac{c_{2}}{1-c_{2}}\left(\frac{1}{c_{1}}+1\right) \sqrt{s}\right)}|u(s, y)|^{2} \mathrm{~d} y \mathrm{~d} s\right) \lesssim\left|B^{*}\right| \sup _{z \in E}\left|\mathcal{N}_{\gamma} u(z)\right|^{2},
$$

for some $\gamma \geq 4$ depending only on $c_{1}, c_{2}$. In the same way, using Proposition 3.6, we also have that

$$
\begin{aligned}
I_{6} & \lesssim \sum_{k=0}^{\infty} \int_{r_{k}^{2}\left(\frac{1}{c_{2}}-1\right)^{2}}^{4 r_{k}^{2}\left(\frac{1}{c_{1}}+1\right)^{2}} \int_{B\left(x_{k}, \frac{c_{2}}{1-c_{2}} \sqrt{s}\right)}|\nabla u(s, y)|^{2} \mathrm{~d} y \mathrm{~d} s, \\
& \lesssim \sum_{k=0}^{\infty} \int_{r_{k}^{2}\left(\frac{1}{c_{2}}-1\right)^{2} / 2}^{4 r_{k}^{2}\left(\frac{1}{c_{1}}+1\right)^{2}} \int_{B\left(x_{k}, \frac{2 c_{2}}{1-c_{2}} \sqrt{s}\right)}|u(s, y)|^{2} \mathrm{~d} y \mathrm{~d} s \lesssim\left|B^{*}\right| \sup _{z \in E}\left|\mathcal{N}_{\gamma^{\prime}} u(z)\right|^{2},
\end{aligned}
$$


for some $\gamma^{\prime} \geq \gamma$ depending only on $c_{1}, c_{2}$. Now fix $\beta=\gamma^{\prime}$. Summing all the estimates, and taking limits as $\varepsilon \rightarrow 0$ and $R \rightarrow \infty$, we have the following:

$$
\int_{E^{*}} \int_{0}^{\infty} f_{B\left(x, \frac{\sqrt{t}}{2}\right)}|\nabla u(t, y)|^{2} \mathrm{~d} y \mathrm{~d} t \mathrm{~d} x \lesssim\left|B^{*}\right| \sigma^{2}+\int_{E}\left|\mathcal{N}_{\beta} u(z)\right|^{2} \mathrm{~d} z .
$$

Now we consider the distribution functions defined by

$$
\begin{aligned}
& g_{S}(\sigma)=\left|\left\{x \in \mathbb{R}^{n} ;\left(\int_{0}^{\infty} f_{B\left(x, \frac{\sqrt{t}}{2}\right)}|\nabla u(t, y)|^{2} \mathrm{~d} y \mathrm{~d} t\right)^{\frac{1}{2}}>\sigma\right\}\right|, \\
& g_{N}(\sigma)=\left|\left\{x \in \mathbb{R}^{n} ; \mathcal{N}_{\beta} u(x)>\sigma\right\}\right| .
\end{aligned}
$$

We have that $\left|B^{*}\right| \lesssim|B|=g_{N}(\sigma)$, and that

$$
\int_{E}\left|\mathcal{N}_{\beta} u(x)\right|^{2} \mathrm{~d} x \leq 2 \int_{0}^{\sigma} t g_{N}(t) \mathrm{d} t .
$$

This implies

$$
\begin{aligned}
g_{S}(\sigma) & \lesssim\left|B^{*}\right|+\frac{1}{\sigma^{2}} \int_{E} \int_{0}^{\infty} f_{B\left(x, \frac{\sqrt{t}}{2}\right)}|\nabla u(t, y)|^{2} \mathrm{~d} y \mathrm{~d} t \mathrm{~d} x \\
& \lesssim\left|B^{*}\right|+\frac{1}{\sigma^{2}} \int_{E^{*}}\left|\mathcal{N}_{\beta} u(x)\right|^{2} \mathrm{~d} x \lesssim g_{N}(\sigma)+\int_{0}^{\sigma} t g_{N}(t) \mathrm{d} t .
\end{aligned}
$$

Therefore, as $p<2$,

$$
\begin{aligned}
\int_{0}^{\infty} \sigma^{p-1} g_{S}(\sigma) \mathrm{d} \sigma & \lesssim \int_{0}^{\infty} \sigma^{p-1} g_{N}(\sigma) \mathrm{d} \sigma+\int_{0}^{\infty} \sigma^{p-3} \int_{0}^{\sigma} t g_{N}(t) \mathrm{d} t \mathrm{~d} \sigma \\
& \lesssim \int_{0}^{\infty} \sigma^{p-1} g_{N}(\sigma) \mathrm{d} \sigma
\end{aligned}
$$

and thus $\nabla u \in T^{p, 2}$ with $\|\nabla u\|_{T^{p, 2}} \lesssim\left\|\mathcal{N}_{\beta} u\right\|_{L^{p}} \lesssim\|u\|_{X^{p}}$.

To finish the proof, we check that $(s, x) \mapsto u\left(s^{2}, x\right) \chi^{2}(s, x) \in L^{2}\left(\varepsilon, 2 R ; H^{1}\left(\mathbb{R}^{n}\right)\right)$. We begin by checking $\int_{\varepsilon}^{2 R} \int_{\mathbb{R}^{n}}\left|u\left(s^{2}, x\right) \chi^{2}(s, x)\right|^{2} \mathrm{~d} s \mathrm{~d} x<\infty$, with constants that depend on $\varepsilon, R$. Indeed, we may split $[\varepsilon, 2 R]$ into a finite number of intervals $\left[\delta^{2}, \beta^{2} \delta^{2}\right]$. For each of them, arguing as for $I_{1}$, we obtain a bound $\int_{E}\left|\mathcal{N}_{\beta} u(x)\right|^{2} \mathrm{~d} x$. By definition of $E$ and $p<2$, this does not exceed $\sigma^{2-p} \int_{\mathbb{R}^{n}}\left|\mathcal{N}_{\beta} u(x)\right|^{p} \mathrm{~d} x<\infty$. Next, $\int_{\varepsilon}^{2 R} \int_{\mathbb{R}^{n}}\left|\nabla\left(u\left(s^{2}, x\right) \chi^{2}(s, x)\right)\right|^{2} \mathrm{~d} s \mathrm{~d} x$ is estimated similarly, using Proposition 3.6 as for $I_{2}$.

\subsection{Controlling the square function by the maximal function for $p \in[2, \infty]$}

Theorem 7.4. Let $u$ be a global weak solution of (1.1). Let $p \in[2, \infty]$. If $u \in X^{p}$, then $\nabla u \in T^{p, 2}$ with $\|\nabla u\|_{T^{p, 2}} \lesssim\|u\|_{X^{p}}$ and implicit constant independent of $u$.

Proof. Step 1: In this step we prove the result for $p=2$. Let $R, \varepsilon>0$ with $R \geq \frac{1}{\sqrt{\varepsilon}}$. Let $\chi \in \mathscr{C}^{\infty}((0, \infty),[0,1])$ be supported in $\left[\varepsilon, \frac{1}{\varepsilon}\right]$, and such that, for some constant $C>0$,

$$
\chi(t)=1 \quad \forall t \in\left[2 \varepsilon, \frac{1}{2 \varepsilon}\right], \quad\left|\chi^{\prime}(t)\right| \leq \frac{C}{\varepsilon} \quad \forall t \in[\varepsilon, 2 \varepsilon], \quad\left|\chi^{\prime}(t)\right| \leq C \varepsilon \quad \forall t \in\left[\frac{1}{2 \varepsilon}, \frac{1}{\varepsilon}\right] .
$$

Let $\theta \in \mathscr{C}^{\infty}\left(\mathbb{R}^{n},[0,1]\right)$ be supported in $B(0,2 R)$ and such that

$$
\theta(x)=1 \quad \forall x \in B(0, R), \quad|\nabla \theta(x)| \leq \frac{C}{R} \quad \forall x \in B(0,2 R) \backslash B(0, R) .
$$


For $M>0$, define $\phi_{M}(t, x)=e^{t \Delta}\left(1_{B(0, M)}\right)(x)$ for all $(t, x) \in(0, \infty) \times \mathbb{R}^{n}$. Remark that $\phi_{M}$ is smooth, that $\phi_{M}(t, x)$ increases as $M$ increases with $\phi_{M}(t, x) \underset{M \rightarrow \infty}{\longrightarrow} 1$ for all $(t, x) \in$ $(0, \infty) \times \mathbb{R}^{n}$, and that

$$
\left\|\nabla \phi_{M}\right\|_{T^{\infty, 2}} \sim\left\|1_{B(0, M)}\right\|_{B M O} \leq 2, \quad\left\|\phi_{M}\right\|_{L^{\infty}\left(L^{\infty}\right)} \leq 1 .
$$

Let $R_{0} \geq 4 R$ and set $\phi=\phi_{R_{0}}$. We want to show

$$
I:=\int_{0}^{\infty} \int_{\mathbb{R}^{n}}|\nabla u(t, x)|^{2} \phi(t, x)^{2} \theta(x)^{2} \chi(t)^{2} \mathrm{~d} x \mathrm{~d} t \lesssim\|u\|_{X^{2}}^{2}=: J,
$$

independently of $u, R_{0}, R$ and $\varepsilon$. Indeed, if this is the case, then we can let $R_{0} \rightarrow \infty$ first by monotone convergence, which implies a control of $\int_{2 \varepsilon}^{1 / 2 \varepsilon} \int_{B(0, R)}|\nabla u(t, x)|^{2} \mathrm{~d} x \mathrm{~d} t$ and it suffices to let $R \rightarrow \infty$ and $\varepsilon \rightarrow 0$.

Let $a=\varepsilon, b=1 / \varepsilon$ and $\Omega=B(0,2 R)$. Let $\psi=\phi \theta \chi$ (we forget the variables to keep the notation reasonable) and remark that $u \psi^{2} \in L^{2}\left(a, b ; H_{0}^{1}(\Omega)\right)$. According to Remark 3.7, we can use this as a test function to obtain

$$
\left.\int_{0}^{\infty} \int_{\mathbb{R}^{n}} A(t, \cdot) \nabla u(t, x) \cdot \overline{\nabla\left(u \psi^{2}\right)(t, x)}\right\rangle \mathrm{d} t \mathrm{~d} x=-\int_{0}^{\infty}\left\langle\partial_{t} u(t, \cdot), u \psi^{2}(t, \cdot)\right\rangle \mathrm{d} t .
$$

From ellipticity,

$$
\begin{aligned}
I \lesssim & \left|\int_{0}^{\infty} \int_{\mathbb{R}^{n}} A(t, x) \nabla u(t, x) \cdot \overline{\nabla\left(u \psi^{2}\right)(t, x)} \mathrm{d} x \mathrm{~d} t\right| \\
& +\left|\int_{0}^{\infty} \int_{\mathbb{R}^{n}} A(t, x) \nabla u(t, x) \cdot \nabla\left(\psi^{2}\right)(t, x) \overline{u(t, x)} \mathrm{d} x \mathrm{~d} t\right| \\
& \lesssim I_{1}+I_{2}+I_{3},
\end{aligned}
$$

where

$$
\begin{aligned}
& I_{1}=\left|\int_{0}^{\infty}\left\langle\partial_{t} u(t, \cdot),\left(u \psi^{2}\right)(t, \cdot)\right\rangle \mathrm{d} t\right|, \\
& I_{2}=\left|\int_{0}^{\infty} \int_{\mathbb{R}^{n}} A(t, x) \nabla u(t, x) \cdot \phi(t, x) \nabla \phi(t, x) \theta^{2}(x) \chi^{2}(t) \overline{u(t, x)} \mathrm{d} x \mathrm{~d} t\right|, \\
& I_{3}=\mid \int_{0}^{\infty} \int_{\mathbb{R}^{n}} A(t, x) \nabla u(t, x) \cdot \phi^{2}(t, x) \theta(x) \nabla \theta(x) \chi^{2}(t) \overline{u(t, x)} \mathrm{d} x \mathrm{~d} t .
\end{aligned}
$$

To estimate $I_{1}$, we decompose further and obtain $I_{1} \lesssim I_{1,1}+I_{1,2}+I_{1,3}$, where (forgetting to write the $t$ variable)

$$
\begin{aligned}
& I_{1,1}=\left|\int_{0}^{\infty}\left\langle\partial_{t}(u \theta \phi \chi), u \theta \phi \chi\right\rangle \mathrm{d} t\right|, \\
& I_{1,2}=\left|\int_{0}^{\infty}\left\langle u \theta \phi \chi \cdot \theta \chi \partial_{t} \phi, u\right\rangle \mathrm{d} t\right|, \\
& I_{1,3}=\left|\int_{0}^{\infty}\left\langle u \theta \phi \chi \cdot \theta \phi \chi^{\prime}, u\right\rangle d t\right| .
\end{aligned}
$$

In the first integral, the inner product is the $H^{-1}(\Omega), H_{0}^{1}(\Omega)$ duality so that we can use Lions' theorem again and we have that $I_{1,1}=\frac{1}{2} \int_{0}^{\infty} \partial_{t}\|u(t, .) \theta \phi(t, .) \chi(t)\|_{2}^{2} \mathrm{~d} t=0$. In the other two integrals, the inner product can be rexpressed with the $L^{2}$ duality. To estimate 
$I_{1,2}$, remark first that, if $g_{t}$ denotes the standard heat kernel defined by $g_{t}(z)=(\pi t)^{-\frac{n}{2}} e^{-\frac{|z|^{2}}{4 t}}$, we have for all $x \in B(0,2 R)$ and for all $t>0$,

$$
\begin{aligned}
\left|\partial_{t} \phi(t, x)\right| & \lesssim\left|\int_{\mathbb{R}^{n}} \partial_{t} g_{t}(x-y) \mathbb{1}_{B\left(0, R_{0}\right)}(y) \mathrm{d} y\right| \\
& =\left|\int_{\mathbb{R}^{n}} \partial_{t} g_{t}(x-y) \mathbb{1}_{B\left(0, R_{0}\right)^{c}}(y) \mathrm{d} y\right| \\
& \leq C \int_{\mathbb{R}^{n}} t^{-n / 2-1} e^{-\frac{R^{2}}{4 t}} e^{-\frac{c|x-y|^{2}}{4 t}} \mathrm{~d} y \lesssim R^{-2} .
\end{aligned}
$$

In this calculation, we used $|x-y| \geq R_{0}-2 R \geq 2 R$, so that $|x-y|^{2} \geq \frac{|x-y|^{2}}{4}+R^{2}$. Now define $k_{\varepsilon} \in \mathbb{N}$ such that $2^{k_{\varepsilon}} \leq \frac{1}{\varepsilon^{2}}<2^{k_{\varepsilon}+1}$. We have the following

$$
I_{1,2} \lesssim R^{-2} \sum_{k=0}^{k_{\varepsilon}} \int_{2^{k} \varepsilon}^{2^{k+1} \varepsilon} \int_{\mathbb{R}^{n}}|u(t, x)|^{2} \mathrm{~d} x \mathrm{~d} t \lesssim R^{-2} \sum_{k=0}^{k_{\varepsilon}} 2^{k} \varepsilon\|u\|_{X^{2}}^{2} \lesssim\|u\|_{X^{2}}^{2},
$$

using $R^{-2} \varepsilon^{-1} \leq 1$. Moreover

$$
I_{1,3} \lesssim f_{\varepsilon}^{2 \varepsilon} \int_{\mathbb{R}^{n}}|u(t, x)|^{2} \mathrm{~d} x \mathrm{~d} t+f_{\frac{1}{2 \varepsilon}}^{\frac{1}{\varepsilon}} \int_{\mathbb{R}^{n}}|u(t, x)|^{2} \mathrm{~d} x \mathrm{~d} t \lesssim\|u\|_{X^{2}}^{2},
$$

since $\left|\chi^{\prime}(t)\right| \leq \frac{C}{\varepsilon}$ for all $t \in[\varepsilon, 2 \varepsilon]$, and $\left|\chi^{\prime}(t)\right| \leq C \varepsilon$ for all $t \in\left[\frac{1}{2 \varepsilon}, \frac{1}{\varepsilon}\right]$.

To estimate $I_{2}$ we use Cauchy-Schwarz inequality, Harnack inequalities for each $\partial_{x_{j}} \phi$, $j=1, \ldots, n$, and Carleson inequality (see [15, Proposition 3]) to obtain

$$
\begin{aligned}
I_{2} & \lesssim\left(\int_{0}^{\infty} \int_{\mathbb{R}^{n}}|\nabla u(t, x)|^{2} \theta(x)^{2} \phi(t, x)^{2} \chi(t)^{2} \mathrm{~d} x \mathrm{~d} t\right)^{\frac{1}{2}}\left(\int_{0}^{\infty} \int_{\mathbb{R}^{n}}|u(t, x)|^{2}|\nabla \phi(t, x)|^{2} \mathrm{~d} x \mathrm{~d} t\right)^{\frac{1}{2}} \\
& =I^{\frac{1}{2}}\left(\int_{0}^{\infty} \int_{\mathbb{R}^{n}}\left(f_{t}^{2 t} f_{B(x, \sqrt{t})}|u(s, y)|^{2}|\nabla \phi(s, y)|^{2} \mathrm{~d} y \mathrm{~d} s\right) \mathrm{d} x \mathrm{~d} t\right)^{\frac{1}{2}} \\
& \lesssim I^{\frac{1}{2}}\left(\int_{0}^{\infty} \int_{\mathbb{R}^{n}}\left(f_{t}^{2 t} f_{B(x, \sqrt{t})}|u(s, y)|^{2} \mathrm{~d} y \mathrm{~d} s\right)|\nabla \phi(t, x)|^{2} \mathrm{~d} x \mathrm{~d} t\right)^{\frac{1}{2}} \\
& \lesssim I^{\frac{1}{2}}\left(\int_{\mathbb{R}^{n}} \sup _{(t, x) \in \Gamma_{z}}\left(f_{t}^{2 t} f_{B(x, \sqrt{t})}|u(s, y)|^{2} \mathrm{~d} y \mathrm{~d} s\right) \mathrm{d} z\|\nabla \phi\|_{T^{\infty, 2}}^{2}\right)^{\frac{1}{2}} \lesssim I^{\frac{1}{2}} J^{\frac{1}{2}} .
\end{aligned}
$$

For $I_{3}$, we have by the Cauchy-Schwarz inequality and $R^{-2} \varepsilon^{-1} \leq 1$,

$$
\begin{aligned}
I_{3} & \lesssim I^{\frac{1}{2}}\left(\int_{0}^{\infty} \int_{\mathbb{R}^{n}}|u(t, x)|^{2}|\nabla \theta(x)|^{2} \phi(t, x)^{2} \chi(t)^{2} \mathrm{~d} x \mathrm{~d} t\right)^{\frac{1}{2}} \lesssim \frac{I^{\frac{1}{2}}}{R}\left(\int_{0}^{\infty} \int_{\mathbb{R}^{n}}|u(t, x)|^{2} \chi(t)^{2} \mathrm{~d} x \mathrm{~d} t\right)^{\frac{1}{2}} \\
& \lesssim \frac{I^{\frac{1}{2}}}{R}\left(\sum_{k=0}^{k_{\varepsilon}} 2^{k_{\varepsilon}} f_{2^{k} \varepsilon}^{2^{k+1} \varepsilon} \int_{\mathbb{R}^{n}} f_{B\left(x, \sqrt{2^{k} \varepsilon}\right)}|u(t, y)|^{2} \mathrm{~d} y \mathrm{~d} t \mathrm{~d} x\right)^{\frac{1}{2}} \lesssim \frac{I^{\frac{1}{2}}}{R}\left(2^{k_{\varepsilon}} \varepsilon\right)^{\frac{1}{2}} J^{\frac{1}{2}} \leq I^{\frac{1}{2}} J^{\frac{1}{2}} .
\end{aligned}
$$

Combining all the estimates, we have that $I \lesssim I^{\frac{1}{2}} J^{\frac{1}{2}}+J$. As $I<\infty$ by definition, we thus conclude that $I \lesssim J$ as desired.

Step 2: In this step we prove the result for $2<p \leq \infty$ essentially by establishing a local version of Step 1. Fix $x_{0} \in \mathbb{R}^{n}, R_{0}, R>0$ with $R_{0} \geq 4 R, \varepsilon>0$ and consider $\chi \in \mathscr{C}^{\infty}(\mathbb{R},[0,1])$ supported in $\left[\varepsilon, 4 R^{2}\right]$ such that, for some $C>0$,

$$
\chi(t)=1 \quad \forall t \in\left[2 \varepsilon, R^{2}\right], \quad\left|\chi^{\prime}(t)\right| \leq \frac{C}{\varepsilon} \quad \forall t \in[\varepsilon, 2 \varepsilon], \quad\left|\chi^{\prime}(t)\right| \leq \frac{C}{R^{2}} \quad \forall t \in\left[R^{2}, 4 R^{2}\right] .
$$


We define $\phi(t, x)=e^{t \Delta}\left(1_{B\left(x_{0}, R_{0}\right)}\right)(x)$ for all $(t, x) \in[0, \infty) \times \mathbb{R}^{n}$. We also let $\theta \in \mathscr{C}^{\infty}\left(\mathbb{R}^{n},[0,1]\right)$ be supported in $B\left(x_{0}, 2 R\right)$ and such that

$$
\theta(x)=1 \quad \forall x \in B\left(x_{0}, R\right), \quad|\nabla \theta(x)| \leq \frac{C}{R} \quad \forall x \notin B\left(x_{0}, R\right) .
$$

Defining

$$
\begin{aligned}
& I=\int_{0}^{\infty} \int_{\mathbb{R}^{n}}|\nabla u(t, x)|^{2} \phi(t, x)^{2} \theta(x)^{2} \chi(t)^{2} \mathrm{~d} t \mathrm{~d} x, \\
& J=\left\|(t, x) \mapsto \mathbb{1}_{B\left(x_{0}, 2 R\right)}(x) \mathbb{1}_{\left(0,4 R^{2}\right)}(t) u(t, x)\right\|_{X^{2}}^{2},
\end{aligned}
$$

we only have to show that $I \lesssim J$ with implicit constants independent of $u, x_{0}, \varepsilon, R, R_{0}$. Indeed, if this holds, then, taking the limit as $R_{0} \rightarrow \infty$ and then as $\varepsilon \rightarrow 0$,

$$
\begin{aligned}
\int_{0}^{R^{2}} f_{B\left(x_{0}, R\right)} & |\nabla u(t, x)|^{2} \mathrm{~d} x \mathrm{~d} t \\
& \lesssim R^{-n} \int_{\mathbb{R}^{n}}\left(\sup _{\delta>0} f_{\delta}^{2 \delta} f_{B(x, \sqrt{\delta})}|u(t, y)|^{2} 1_{\left(0,4 R^{2}\right)}(t) 1_{B\left(x_{0}, 2 R\right)}(y) \mathrm{d} y \mathrm{~d} t\right)^{2} \mathrm{~d} x \\
& \lesssim R^{-n} \int_{B\left(x_{0}, 4 R\right)}|\tilde{N} u(x)|^{2} \mathrm{~d} x \lesssim \inf _{y \in B\left(x_{0}, 4 R\right)} M\left((\tilde{N} u)^{2}\right)(y)
\end{aligned}
$$

where $M$ is the Hardy-Littlewood operator on $\mathbb{R}^{n}$.

Define $C(F)(y)=\sup _{B \ni y}\left(\int_{B} \int_{0}^{r^{2}}|F(t, x)|^{2} \mathrm{~d} t \mathrm{~d} x\right)^{\frac{1}{2}}$. Thus if $y \in \mathbb{R}^{n}$ by taking the supremum over all $B=B\left(x_{0}, R\right) \ni y$, we have shown

$$
C(|\nabla u|)(y) \lesssim\left[M\left((\tilde{N} u)^{2}\right)\right]^{\frac{1}{2}}(y) .
$$

As $p>2$, using the parabolic version of $[15$, Theorem 3, (2)] and the maximal theorem, we obtain the conclusion.

We proceed as in Step 1, estimating $I \lesssim I_{1,1}+I_{1,2}+I_{1,3}+I_{2}+I_{3}$, where

$$
\begin{aligned}
I_{1,1} & =\left|\int_{0}^{\infty}\left\langle u, \partial_{t}(u \theta \phi \chi) \theta \phi \chi\right\rangle \mathrm{d} t\right|=0, \\
I_{1,2} & =\left|\int_{0}^{\infty}\left\langle u, u \theta \phi \chi \cdot \theta \chi \partial_{t} \phi\right\rangle \mathrm{d} t\right|, \\
I_{1,3} & =\left|\int_{0}^{\infty}\left\langle u, u \theta \phi \chi \cdot \theta \phi \chi^{\prime}\right\rangle \mathrm{d} t\right|, \\
I_{2} & =\left|\int_{0}^{\infty} \int_{\mathbb{R}^{n}} A(t, x) \nabla u(t, x) \cdot \phi(t, x) \nabla \phi(t, x) \theta^{2}(x) \chi^{2}(t) \overline{u(t, x)} \mathrm{d} x \mathrm{~d} t\right|, \\
I_{3} & =\left|\int_{0}^{\infty} \int_{\mathbb{R}^{n}} A(t, x) \nabla u(t, x) \cdot \phi^{2}(t, x) \theta(x) \nabla \theta(x) \chi^{2}(t) \overline{u(t, x)} \mathrm{d} x \mathrm{~d} t\right| .
\end{aligned}
$$

Using $\left|\partial_{t} \phi(t, x)\right| \lesssim R_{0}^{-1}$, we have that

$$
I_{1,2} \lesssim \int_{0}^{R^{2}} \int_{B\left(x_{0}, 2 R\right)}|u(t, x)|^{2}\left|\partial_{t} \phi(t, x)\right| \mathrm{d} x \mathrm{~d} t \lesssim \frac{R^{2}}{R_{0}^{2}} \int_{\mathbb{R}^{n}} \tilde{N}\left(1_{B\left(x_{0}, 2 R\right)} u\right)(x)^{2} \mathrm{~d} x \lesssim J,
$$

and using the properties of $\chi$ and $\theta$, we also obtain

$$
I_{1,3} \lesssim f_{\varepsilon}^{2 \varepsilon} \int_{\mathbb{R}^{n}}\left|1_{B\left(x_{0}, 2 R\right)}(x) u(t, x)\right|^{2} \mathrm{~d} x \mathrm{~d} t+f_{R^{2}}^{4 R^{2}} \int_{\mathbb{R}^{n}}\left|1_{B\left(x_{0}, 2 R\right)}(x) u(t, x)\right|^{2} \mathrm{~d} x \mathrm{~d} t \lesssim J
$$


Next, as in Step 1, we also have that $I_{2} \lesssim I^{\frac{1}{2}} J^{\frac{1}{2}}$. Finally, by Cauchy-Schwarz inequality

$$
\begin{aligned}
I_{3} & \lesssim \frac{I^{\frac{1}{2}}}{R}\left(\int_{\mathbb{R}^{n}} \int_{\varepsilon}^{4 R^{2}}\left|1_{B\left(x_{0}, 2 R\right)}(x) u(t, x)\right|^{2} \mathrm{~d} x \mathrm{~d} t\right)^{\frac{1}{2}} \\
& \lesssim \frac{I^{\frac{1}{2}}}{R}\left(\int_{\mathbb{R}^{n}} \sum_{k=-2}^{\infty} 2^{-k} R^{2} f_{2^{-k-1} R^{2}}^{2^{-k} R^{2}} f_{B\left(x, R 2^{-\frac{k}{2}}\right)}\left|1_{B\left(x_{0}, 2 R\right)}(x) u(t, x)\right|^{2} \mathrm{~d} x \mathrm{~d} t \mathrm{~d} y\right)^{\frac{1}{2}} \lesssim I^{\frac{1}{2}} J^{\frac{1}{2}}
\end{aligned}
$$

This concludes Step 2.

\subsection{Consequences}

We have obtained comparison results only for solutions with $L^{2}$ data. We can remove this constraint as follows.

Corollary 7.5. Let $1<p<\infty$. Assume that, for all $f \in L^{p}\left(\mathbb{R}^{n}\right)$, the problem

$$
\partial_{t} u=\operatorname{div} A \nabla u, \quad u \in X^{p}, \quad \lim _{t \rightarrow 0} u(t, \cdot)=f \text { in } L_{\mathrm{loc}}^{2},
$$

is well-posed. Then the solution satisfies

$$
\|\tilde{N}(u)\|_{L^{p}} \sim\|\nabla u\|_{T^{p, 2}} \sim\|f\|_{L^{p}} .
$$

Remark 7.6. We have well-posedness in $X^{p}$ for $p \geq 2$ by Corollaries 5.5 and 7.2. For $p<2$, we have well-posedness in $X^{p}$ under the assumptions of either Corollary 5.10, or Theorem 6.9, or Theorem 6.14.

Proof. Let $u \in X^{p}$ be a global weak solution of (1.1). By well-posedness in $X^{p}$, we know that $u(t, \cdot)=\Gamma(t, 0) f$ for a unique $f \in L^{p}\left(\mathbb{R}^{n}\right)$, for all $t>0$. Moreover $\|\tilde{N}(u)\|_{p} \sim\|f\|_{p}$. Consider an approximation $f_{k} \in L^{p}\left(\mathbb{R}^{n}\right) \cap L^{2}\left(\mathbb{R}^{n}\right)$ converging to $f$, and let $u_{k}$ be the corresponding solution. We have that $\left\|\tilde{N}\left(u_{k}\right)\right\|_{p} \sim\left\|\nabla u_{k}\right\|_{T^{p, 2}}$ combining Proposition 7.1 and Theorems 7.3 and 7.4. The conclusion follows from taking the limit as $k \rightarrow \infty$.

We wish to make a connection with old ideas in the topic such as those of Nash, as explained in [19]. Assume $A$ has real coefficients. Then for $h \in \mathscr{C}_{c}^{\infty}\left(\mathbb{R}^{n}\right), h \geq 0, u(t)=$ $\Gamma(t, 0) h$, and $2 \leq p<\infty$, one has (if the coefficients are smooth to ease justifications)

$$
\frac{\mathrm{d}}{\mathrm{d} t}\|u(t)\|_{L^{p}}^{p}=p(p-1) \int_{\mathbb{R}^{n}} u(t, x)^{p-2} A(t, x) \nabla u(t, x) \cdot \nabla u(t, x) \mathrm{d} x
$$

and in particular

$$
\|h\|_{L^{p}}^{p}-\|u(t)\|_{L^{p}}^{p}=p(p-1) \int_{0}^{t} \int_{\mathbb{R}^{n}} u(s, x)^{p-2} A(s, x) \nabla u(s, x) \cdot \nabla u(s, x) \mathrm{d} x \mathrm{~d} s .
$$

This yields the finiteness of the integral when $t \rightarrow \infty$, which is equal to $\|h\|_{L^{p}}^{p}$ as $\|u(t)\|_{L^{p}} \rightarrow$ 0 . Note that for $p=2$, we recover the energy equality

$$
\|h\|_{L^{2}}^{2}=2 \int_{0}^{\infty} \int_{\mathbb{R}^{n}} A(s, x) \nabla u(s, x) \cdot \nabla u(s, x) \mathrm{d} x \mathrm{~d} s
$$


which motivated our approach to the energy space. Our observation is that for $p>2$ and arbitrary global weak solutions with $\left\|u^{*}\right\|_{L^{p}}<\infty$ (which, as we have shown, are of the form $u(t)=\Gamma(t, 0) h$ for a unique $\left.h \in L^{p}\left(\mathbb{R}^{n}\right)\right)$ we have

$$
I:=\int_{0}^{\infty} \int_{\mathbb{R}^{n}}|u(s, x)|^{p-2} A(s, x) \nabla u(s, x) \cdot \nabla u(s, x) \mathrm{d} x \mathrm{~d} s \lesssim\left\|u^{*}\right\|_{L^{p}}^{p} .
$$

Indeed, using the boundedness of $A$,

$$
\begin{aligned}
\int_{0}^{\infty} \int_{\mathbb{R}^{n}}|u(s, x)|^{p-2} A(s, x) \nabla u(s, x) \cdot \nabla u(s, x) \mathrm{d} x \mathrm{~d} s & \lesssim \int_{\mathbb{R}^{n}} C(|\nabla u|)(x)^{2} u^{*}(x)^{p-2} \mathrm{~d} x \\
& \leq\|C(|\nabla u|)\|_{L^{p}}^{2}\left\|u^{*}\right\|_{L^{p}}^{p-2}
\end{aligned}
$$

by Hölder's inequality in the last line and, in the first, [15, Proposition 3] adapted to parabolic scaling, with $C(f)(x)$ is the supremum of $\left(\int_{0}^{r^{2}} f_{B} f^{2}(t, y) \mathrm{d} y \mathrm{~d} t\right)^{\frac{1}{2}}$ taken over all balls $B, r$ being the radius, that contain $x$. Next, by [15, Theorem 3, (b)], we have $\|C(|\nabla u|)\|_{L^{p}} \lesssim\|\nabla u\|_{T^{p, 2}}$. Finally we conclude using our Theorem 7.4.

It is not clear at all that integrals of the form $I$ can play a role when considering complex equations. In particular, (7.1) does not hold in this case. However, (modified) maximal functions and Lusin area functionals remain valid tools, as we have just demonstrated.

\section{Fatou type results}

Our well-posedness results imply convergence in $L_{l o c}^{2}\left(\mathbb{R}^{n}\right)$ or in $L^{p}\left(\mathbb{R}^{n}\right)$ sense for some $p$ to the initial value. Here, we address almost everywhere convergence issues. As weak solutions may not be locally bounded, we replace the pointwise (parabolic) non-tangential convergence by the convergence of (parabolic) Whitney averages.

Theorem 8.1. Let $A$ be as in (1.2). Let $f \in L^{2}\left(\mathbb{R}^{n}\right)$ and $u(t, \cdot)=\Gamma(t, 0) f$. Then for almost every $x \in \mathbb{R}^{n}$, we have convergence of the Whitney averages

$$
\lim _{\delta \rightarrow 0} f_{\frac{\delta}{2}}^{\delta} f_{B(x, \sqrt{\delta})}|u(t, y)-f(x)|^{2} \mathrm{~d} y \mathrm{~d} t=0,
$$

as well as of the slice averages

$$
\lim _{\delta \rightarrow 0} f_{B(x, \sqrt{\delta})}|u(\delta, y)-f(x)|^{2} \mathrm{~d} y=0 .
$$

In particular, for almost every $x \in \mathbb{R}^{n}$,

$$
\lim _{\delta \rightarrow 0} f_{\frac{\delta}{2}}^{\delta} f_{B(x, \sqrt{\delta})} u(t, y) \mathrm{d} y \mathrm{~d} t=\lim _{\delta \rightarrow 0} f_{B(x, \sqrt{\delta})} u(\delta, y) \mathrm{d} y=f(x)
$$

Proof. Considering $f(x)$ as a constant, the convergence of the slice averages follows from the local estimates in Proposition 3.6 and the convergence of the Whitney averages.

To show the convergence of the Whitney averages, we use again the Duhamel formula (6.2) with $L=-\Delta$, which reads, for almost every $(t, y) \in \mathbb{R}_{+}^{n+1}$,

$$
u(t, y)=e^{t \Delta} f(y)+\mathcal{R}_{-\Delta}((A-I) \nabla u)(t, y)=e^{t \Delta} f(y)+v(t, y) .
$$


Recall that $v(t, y)$ only involves $(A-I) \nabla u$ at times between 0 and $t$. Thus, the proof of the boundedness from $T^{2,2}=L^{2}\left(L^{2}\right)$ to $X^{2}$ from Proposition 2.12 shows that

$$
\int_{\mathbb{R}^{n}} \sup _{\delta \leq \varepsilon} f_{\frac{\delta}{2}}^{\delta} f_{B(x, \sqrt{\delta})}|v(t, y)|^{2} \mathrm{~d} y \mathrm{~d} t \mathrm{~d} x \lesssim\|(A-I) \nabla u\|_{L^{2}\left(0, \varepsilon ; L^{2}\right)}^{2} .
$$

The right-hand side converges to 0 with $\varepsilon \rightarrow 0$ as $\|\nabla u\|_{L^{2}\left(L^{2}\right)} \sim\|f\|_{L^{2}}$. Thus the left-hand side converges to 0 . As the integrand is non negative and non decreasing as a function of $\varepsilon$, it follows that it converges to 0 almost everywhere, that is

$$
f_{\frac{\delta}{2}}^{\delta} f_{B(x, \sqrt{\delta})}|v(t, y)|^{2} \mathrm{~d} y \mathrm{~d} t \underset{\delta \rightarrow 0}{\longrightarrow} 0 \quad \text { almost everywhere. }
$$

Finally, for almost every $x \in \mathbb{R}^{n}$,

$$
f_{\frac{\delta}{2}}^{\delta} f_{B(x, \sqrt{\delta})}\left|e^{t \Delta} f(y)-f(x)\right|^{2} \mathrm{~d} y \mathrm{~d} t \underset{\delta \rightarrow 0}{\longrightarrow} 0
$$

by the known results concerning pointwise non-tangential almost everywhere convergence of the solutions of the heat equation. This completes the proof.

Corollary 8.2. Let $1<p<\infty$. Consider any $A$ as in (1.2) for which (1.1) is well-posed on $X^{p}$ with initial space $L^{p}$ (this holds for $p \geq 2$ and under conditions when $p<2$ ). Let $u$ be a global weak solution in $X^{p}$. Then

$$
f_{\frac{\delta}{2}}^{\delta} f_{B(x, \sqrt{\delta})} u(t, y) \mathrm{d} y \mathrm{~d} t \quad \text { and } \quad f_{B(x, \sqrt{\delta})} u(\delta, y) \mathrm{d} y
$$

converge for almost every $x \in \mathbb{R}^{n}$ to the initial value as $\delta \rightarrow 0$.

Proof. Under our assumption, we know that $u(t, \cdot)=\Gamma(t, 0) f$ for a unique $f \in L^{p}$ for all $t>0$. We invoke the usual argument involving 1) control of the maximal function $\tilde{N}(u)$ and the one for the slice averages in $L^{p}$, and 2) existence of limits almost everywhere for a dense class of $f$, here $L^{p} \cap L^{2}$, from the previous theorem. We skip details.

Remark 8.3. Consider $A$ with real coefficients. This results holds for any $1<p<\infty$ and can be proven by usual arguments, using the pointwise Gaussian upper estimate and the conservation property. For $p=\infty$, one can get convergence under uniform continuity of $f$. For $p=1$, we have not attempted to describe the space $\left\{u \in L_{\mathrm{loc}}^{2}\left(\mathbb{R}_{+}^{n+1}\right) ;\|u\|_{X^{1}}<\infty\right\}$. This would define a Hardy space associated with a non-autonomous equation.

\section{$9 \quad L^{1}$ theory for propagators with kernel bounds}

In this section, we assume that the propagators $\Gamma(t, s)$, for $t>s \geq 0$, have kernel bounds, and that their adjoints have some time regularity. More precisely, we assume the following:

$$
\begin{aligned}
& |k(t, s, x, y)| \leq C(t-s)^{-\frac{n}{2}} e^{-c \frac{|x-y|^{2}}{t-s}}, \\
& \forall h \in \mathscr{C}_{c}\left(\mathbb{R}^{n}\right) \quad\left\|\Gamma\left(t_{0}, s\right)^{*} h-\Gamma\left(t_{0}, s_{0}\right)^{*} h\right\|_{L^{\infty}} \underset{s \rightarrow s_{0}}{\longrightarrow} 0,
\end{aligned}
$$

for some $C, c>0$, all $t_{0}>s_{0} \geq 0$, and almost all $x, y \in \mathbb{R}^{n}$.

See Section 4.3 for a discussion of the first condition. The second condition can be checked for real equations as a consequence of Nash's regularity theorem [32]. 
Lemma 9.1. Assume (9.1). Let $t>s>r \geq 0$. Then

$$
\int_{\mathbb{R}^{n}} k(t, s, x, z) k(s, r, z, y) \mathrm{d} z=k(t, r, x, y),
$$

for almost every $x, y \in \mathbb{R}^{n}$. Moreover,

$$
\int_{\mathbb{R}^{n}} k(t, r, x, y) d x=1
$$

for almost every $y \in \mathbb{R}^{n}$. Finally, for any $f \in L^{1}\left(\mathbb{R}^{n}\right), \tau \mapsto \Gamma(\tau, s) f \in \mathscr{C}_{0}\left([s, \infty), L^{1}\right)$.

Proof. Let $x, y \in \mathbb{R}^{n}$ and define $I(x, y)=\int_{\mathbb{R}^{n}} k(t, s, x, z) k(s, r, z, y) \mathrm{d} z$. Note that the integral converges thanks to (9.1). Next, for $f \in \mathscr{D}\left(\mathbb{R}^{n}\right)$, we have the following for almost all $x \in \mathbb{R}^{n}$

$$
\begin{aligned}
\int_{\mathbb{R}^{n}} I(x, y) f(y) \mathrm{d} y & =\int_{\mathbb{R}^{n}} k(t, s, x, z)\left(\int_{\mathbb{R}^{n}} k(s, r, z, y) f(y) \mathrm{d} y\right) \mathrm{d} z \\
& =\int_{\mathbb{R}^{n}} k(t, s, x, z) \Gamma(s, r) f(z) \mathrm{d} z=\Gamma(t, s) \Gamma(s, r) f(x) \\
& =\Gamma(t, s) f(x)=\int_{\mathbb{R}^{n}} k(t, s, x, y) f(y) \mathrm{d} y .
\end{aligned}
$$

Using (9.1), we have that the other equality is equivalent to $\Gamma(t, r)^{*} 11=11$ almost everywhere. This is proved in the $L_{\text {loc }}^{2}$ sense in Corollary 5.6.

Finally, the strong continuity of $\tau \mapsto \Gamma(\tau, s)$ on $L^{1}\left(\mathbb{R}^{n}\right)$ is proven as follows. As the operators are uniformly bounded on $L^{1}\left(\mathbb{R}^{n}\right)$, it suffices to check continuity for functions in a dense class. Let $f \in L^{\infty}\left(\mathbb{R}^{n}\right)$ be compactly supported, and $M>0$ be such that the support of $f$ is contained $B(0, M)$. Then, using $(9.1),\left\|\Gamma(t, s) f-\Gamma\left(t^{\prime}, s\right) f\right\|_{L^{1}\left(B(0, M)^{c}\right)} \rightarrow 0$ when $M \rightarrow \infty$ uniformly for $t, t^{\prime}$ in any bounded set of $[s, \infty)$. So fix $t \geq s$ and take $t^{\prime} \geq s$ with $\left|t-t^{\prime}\right| \leq 1$. Let $\varepsilon>0$ and fix $M>0$ so that $\left\|\Gamma(t, s) f-\Gamma\left(t^{\prime}, s\right) f\right\|_{L^{1}\left(B(0, M)^{c}\right)} \leq \varepsilon$ for all such $t, t^{\prime}$. Since $\left\|\Gamma(t, s) f-\Gamma\left(t^{\prime}, s\right) f\right\|_{L^{1}(B(0, M))} \leq M^{n / 2}\left\|\Gamma(t, s) f-\Gamma\left(t^{\prime}, s\right) f\right\|_{L^{2}(B(0, M))}$, we can use the strong continuity of $\tau \mapsto \Gamma(\tau, s)$ on $L^{2}\left(\mathbb{R}^{n}\right)$ to conclude. The proof for the limit at $\infty$ is similar.

Theorem 9.2. Assume (9.1) and (9.2). For $u \in \mathscr{D}^{\prime}$, the following assertions are equivalent.

$$
\begin{aligned}
& \exists ! u_{0} \in L^{1}\left(\mathbb{R}^{n}\right) \text { such that } u(t, \cdot)=\Gamma(t, 0) u_{0} \text { in } L^{1}\left(\mathbb{R}^{n}\right) \text { for all } t>0 ; \\
& u \text { is a global weak solution of }(1.1) \text { in } L_{w c s}^{\infty}\left(L^{1}\right),
\end{aligned}
$$

where $u \in L_{w c s}^{\infty}\left(L^{1}\right)$ means $u \in L^{\infty}\left(L^{1}\right)$ and there exists a weakly convergent sequence $\left(u\left(t_{j}, .\right)\right)_{j \in \mathbb{N}}$ in $L^{1}\left(\mathbb{R}^{n}\right)$, where $\left(t_{j}\right)_{j \in \mathbb{N}}$ is a sequence of positive reals decreasing to 0 . In this case, $u \in \mathscr{C}_{0}\left([0, \infty), L^{1}\right)$.

Proof. To prove that (9.4) implies (9.5), let $f \in L^{1}\left(\mathbb{R}^{n}\right)$ and define $u:(t, x) \mapsto \Gamma(t, 0) f(x)$ by the intergal formula. By (9.1) and Lemma 9.1, we have that $u \in \mathscr{C}_{0}\left([0, \infty), L^{1}\right)$. It remains to see it is a global weak solution of (1.1). For all $a>0$ and $t \in(a, \infty)$, we have, thanks to (9.1), that there exists $c>0$ such that

$$
\left\|\Gamma\left(\frac{a}{2}, 0\right) f\right\|_{L^{2}} \lesssim\left\|e^{c a \Delta}|f|\right\|_{L^{2}} \lesssim\|f\|_{L^{1}} .
$$


By Lemma 9.1, and density of $L^{1} \cap L^{2}$ in $L^{1}, \Gamma(t, 0) f=\Gamma\left(t, \frac{a}{2}\right) \Gamma\left(\frac{a}{2}, 0\right) f$ for all $t \in(a, \infty)$. This shows that $u$ is a global weak solution of (1.1).

We now turn to the other direction, and assume (9.5). Let $\left(t_{j}\right)_{j \in \mathbb{N}}$ be the sequence of positive reals decreasing to 0 such that $\left(u\left(t_{j}, \cdot\right)\right)_{j \in \mathbb{N}}$ converges weakly in $L^{1}\left(\mathbb{R}^{n}\right)$. Call $f$ its limit. By Proposition 4.3 and Theorem 5.1, for all $j \in \mathbb{N}$ with $t>t_{j}$, and $h \in \mathscr{C}_{c}\left(\mathbb{R}^{n}\right)$, we have that

$$
\int_{\mathbb{R}^{n}} u\left(t_{j}, x\right) \overline{\Gamma\left(t, t_{j}\right)^{*} h(x)} \mathrm{d} x=\int_{\mathbb{R}^{n}} u(t, x) \overline{h(x)} \mathrm{d} x .
$$

Using (9.2) and also that $\left(\left\|u\left(t_{j}, \cdot\right)\right\|_{1}\right)_{j \in \mathbb{N}}$ is a bounded sequence, the left hand side converges to $\int_{\mathbb{R}^{n}} f(x) \overline{\Gamma(t, 0)^{*} h(x)} \mathrm{d} x$. This implies $u(t, \cdot)=\Gamma(t, 0) f$. The uniqueness of $u_{0}=f$ follows by continuity at $t=0$ in $L^{1}\left(\mathbb{R}^{n}\right)$.

We then consider solutions only in $L^{\infty}\left(L^{1}\right)$ and show that such solutions arise from considering Radon measures as initial data. To do so, we need to impose a further condition on the kernel, which is satisfied in the case of real coefficients (see [3, Theorem 9]), again as a consequence of Nash's regularity theorem [32].

For all $t>s \geq 0$ and almost all $x \in \mathbb{R}^{n}, \quad y \mapsto k(t, s, x, y)$ is continuous on $\mathbb{R}^{n}$.

Under (9.1) and (9.6), then for all $t>0$ the integral $\int_{\mathbb{R}^{n}} k(t, 0, x, y) \mathrm{d} \mu(y)$ makes sense in the duality of $\mathscr{C}_{0}\left(\mathbb{R}^{n}\right)$ with the space $\mathscr{M}\left(\mathbb{R}^{n}\right)$ of Radon measures for almost all $x \in \mathbb{R}^{n}$, and belongs to $L^{1}\left(\mathbb{R}^{n}\right)$ as a function of $x$. We call this function $\Gamma(t, 0) \mu$.

Theorem 9.3. Assume (9.1), (9.2) and (9.6). For $u \in \mathscr{D}^{\prime}$, the following assertions are equivalent.

$$
\begin{aligned}
& \exists ! \mu \in \mathscr{M}\left(\mathbb{R}^{n}\right) \text { such that } u(t, \cdot)=\Gamma(t, 0) \mu \text { for all } t>0 ; \\
& u \text { is a global weak solution of }(1.1) \text { in } L^{\infty}\left(L^{1}\right) .
\end{aligned}
$$

In this case, $u \in \mathscr{C}_{0}\left((0, \infty), L^{1}\right)$ and is weakly-star convergent to $\mu$ as $t \rightarrow 0$.

Proof. We first show that (9.7) implies (9.8). Let $\mu$ be a Radon measure and $u(t, \cdot)=$ $\Gamma(t, 0) \mu$ for all $t>0$. By (9.1) and Fubini's theorem, we have that $u \in L^{\infty}\left(L^{1}\right)$. Moreover, for all $a>0$ and $t \in(a, \infty)$, we have that $u\left(\frac{a}{2}, \cdot\right) \in L^{1}\left(\mathbb{R}^{n}\right) \cap L^{\infty}\left(\mathbb{R}^{n}\right)$, and, in particular $u\left(\frac{a}{2}, \cdot\right) \in L^{2}\left(\mathbb{R}^{n}\right)$. By the reproducing formula for the kernels in Lemma 9.1 and Fubini's theorem, $u(t, \cdot)=\Gamma\left(t, \frac{a}{2}\right) u\left(\frac{a}{2}, \cdot\right)$. This shows that $u$ is a weak solution of (1.1) on $\left(\frac{a}{2}, \infty\right)$ for any $a>0$. Hence it is a global weak solution.

Turning to the other direction, we assume (9.8). Let $t_{0}<t$ and $\left(t_{j}\right)_{j \in \mathbb{N}}$ be a sequence of positive reals decreasing to 0 and such that $\left(\int_{\mathbb{R}^{n}} u\left(t_{j}, x\right) \overline{g(x)} \mathrm{d} x\right)_{j \in \mathbb{N}}$ converges for all $g \in$ $\mathscr{C}_{0}\left(\mathbb{R}^{n}\right)$ by Banach-Alaoglu's theorem. Let $\mu \in \mathscr{M}\left(\mathbb{R}^{n}\right)$ be the weak ${ }^{*}$ limit of $\left(u\left(t_{j}, \cdot\right)\right)_{j \in \mathbb{N}}$. Let $g \in \mathscr{C}_{c}\left(\mathbb{R}^{n}\right)$. Using (9.1) and (9.6), we see that $\Gamma\left(t, t_{j}\right)^{*} g \in \mathscr{C}_{0}\left(\mathbb{R}^{n}\right)$, and by (9.2), that

$$
\left\|\Gamma\left(t, t_{j}\right)^{*} g-\Gamma(t, 0)^{*} g\right\|_{L^{\infty}} \underset{j \rightarrow \infty}{\longrightarrow} 0 .
$$

By Proposition 4.3 and Theorem 5.1, we have that

$$
\int_{\mathbb{R}^{n}} u\left(t_{j}, x\right) \overline{\Gamma\left(t, t_{j}\right)^{*} g(x)} \mathrm{d} x=\int_{\mathbb{R}^{n}} u(t, x) \overline{g(x)} \mathrm{d} x
$$

so that taking the limit as $j \rightarrow \infty$, we obtain

$$
\int_{\mathbb{R}^{n}} u(t, x) \overline{g(x)} \mathrm{d} x=\int_{\mathbb{R}^{n}} \overline{\Gamma(t, 0)^{*} g(y)} \mathrm{d} \mu(y)=\int_{\mathbb{R}^{n}} \int_{\mathbb{R}^{n}} k(t, 0, x, y) \overline{g(x)} \mathrm{d} \mu(y) \mathrm{d} x,
$$


and thus

$$
u(t, x)=\int_{\mathbb{R}^{n}} k(t, 0, x, y) \mathrm{d} \mu(y),
$$

for all $t>0$ and almost all $x \in \mathbb{R}^{n}$.

To show that $\mu$ is unique, let $g \in \mathscr{C}_{c}\left(\mathbb{R}^{n}\right)$, and $\varepsilon>0$. By uniform continuity, pick $\delta>0$ such that $|g(x)-g(y)| \leq \varepsilon$ for all $x, y$ such that $|x-y| \leq \delta$. Using (9.1), we have that

$$
\left|\int_{\mathbb{R}^{n}} \int_{B(y, 2 \delta)} k(t, 0, x, y)(g(x)-g(y)) \mathrm{d} x \mathrm{~d} \mu(y)\right| \lesssim \varepsilon\|\mu\|_{\mathscr{M}} .
$$

Moreover, we have that

$$
\left|\int_{\mathbb{R}^{n}} \int_{B(y, 2 \delta)^{c}} k(t, 0, x, y)(g(x)-g(y)) \mathrm{d} x \mathrm{~d} \mu(y)\right| \lesssim\|\mu\|_{\mathscr{M}}\|g\|_{L^{\infty}} e^{-c \frac{\delta^{2}}{t}},
$$

for some constant $c>0$. Therefore,

$$
\lim _{t \rightarrow 0} \int_{\mathbb{R}^{n}} \int_{\mathbb{R}^{n}} k(t, 0, x, y)(g(x)-g(y)) \mathrm{d} x \mathrm{~d} \mu(y)=0 .
$$

By Lemma 9.1, we have that

$\int_{\mathbb{R}^{n}} \int_{\mathbb{R}^{n}} k(t, 0, x, y) g(x) \mathrm{d} x \mathrm{~d} \mu(y)-\int_{\mathbb{R}^{n}} g(y) \mathrm{d} \mu(y)=\int_{\mathbb{R}^{n}} \int_{\mathbb{R}^{n}} k(t, 0, x, y)(g(x)-g(y)) \mathrm{d} x \mathrm{~d} \mu(y)$,

and thus $\int_{\mathbb{R}^{n}} g(x) \mathrm{d} \mu(x)=\lim _{t \rightarrow 0} \int_{\mathbb{R}^{n}} u(t, x) g(x) \mathrm{d} x$, which proves uniqueness of $\mu$.

Finally, the continuity on $(0, \infty)$ and the limit at $\infty$ follow directly from Lemma 9.1 since $u(t, \cdot)=\Gamma(t, s) u(s, \cdot)$ for all $t \geq s>0$ and $u(s, \cdot) \in L^{1}\left(\mathbb{R}^{n}\right)$.

\section{Local results}

We have been interested in global results with scale invariant norms in the interior. As our estimates depend separately on 1) interior representation and 2) taking limits as $t$ tends to 0 , we can formulate well-posedness for larger classes as follows. Let $X$ be one of the spaces where we can prove well-posedness for $Y$ data. For $T>0$, let $X_{T}$ be a local version of $X$, obtained by truncating functions by 0 , i.e. $\|u\|_{X_{T}}=\left\|(t, x) \mapsto u(t, x) \mathbb{1}_{(0, T)}(t)\right\|_{X}$. Let $\mathcal{X}=\cap_{T>0} X_{T}$. Note that functions in $\mathcal{X}$ can have their $X_{T}$ norms growing arbitrarily fast as $T \rightarrow \infty$. Functions with bounded $X_{T}$ norms belong to $X$.

Consider the Cauchy problem for (1.1) on $\mathbb{R}_{+}^{n+1}$ with $u \in \mathcal{X}$ and $u_{0} \in Y$. In each of the following cases, one obtains uniqueness. Since we can construct solutions in $X$, we get a posteriori control at $T \rightarrow \infty$ and the solution belongs to $X$. In other words, arbitrary a priori control on the norms for large times implies a posteriori bounded control. The interested reader can write the precise statements from our explanations.

For $Y=L^{2}\left(\mathbb{R}^{n}\right)$, one can take $X=L^{\infty}\left(L^{2}\right)$ or $X=X^{2}$. In the case $X=L^{2}\left(\dot{H}^{1}\right)$, one takes $X_{T}=L^{2}\left(0, T ; H^{1}\right)$ instead of $L^{2}\left(0, T ; \dot{H}^{1}\right)$.

For $Y=L^{p}\left(\mathbb{R}^{n}\right), 2<p \leq \infty$, one can take $X=X^{p}$ or the space in Proposition 5.7 when $p<\tilde{q}$.

For $Y=L^{p}\left(\mathbb{R}^{n}\right), 1 \leq p<\infty$ and uniform $L^{q}$ bounds on the propagators or estimates on its kernel (see above for the relation between $q$ and $p$ ), one can take $X=L^{\infty}\left(L^{p}\right)$. 
For $Y=L^{p}\left(\mathbb{R}^{n}\right), 1 \leq p<\infty$ and non uniform $L^{q}$ bounds on the propagators or estimates on its kernel (i.e. allowing the $\mathscr{L}\left(L^{q}\right)$ norm of $\Gamma(t, s)$ to grow as $|t-s| \rightarrow \infty$ ), one also obtains well-posedness for $X=L^{\infty}\left(L^{p}\right)$, but the solution has $X_{T}$ norms that may grow at infinity.

For $Y=L^{p}\left(\mathbb{R}^{n}\right), p_{c}<p<2$ and $B V\left(0, T ; L^{\infty}\right)$ coefficients for all $T>0$, we are in the previous situation with $X=L^{\infty}\left(L^{p}\right)$.

We also note that the results $\|\nabla u\|_{T^{p, 2}} \lesssim\|u\|_{X^{p}}$ in Section 7 are easily localisable in time by looking at the proofs. Thus the further estimates using truncated maximal functions apply and one can deduce bounds on truncated Lusin area integrals. However, note that the converse $\|u\|_{X^{p}} \lesssim\|\nabla u\|_{T^{p, 2}}$ is not localisable in time because the right hand side vanishes when the time becomes small.

\section{References}

[1] W. Arendt, R. Chill, C. Seifert, H. Vogt, and J. Voigt, Form methods for evolution equations, and applications, Lecture Notes, available at https://www.mat.tuhh.de/veranstaltungen/isem18/images/5/59/LectureNotes.pdf, 2015. 3.3

[2] D. G. Aronson, Bounds for the fundamental solution of a parabolic equation, Bull. Amer. Math. Soc. 73 (1967), 890-896. 1, 3.4, 3.5, 4.1

[3] D. G. Aronson, Non-negative solutions of linear parabolic equations, Ann. Scuola Norm. Sup. Pisa (3) 22 (1968), 607-694. 1, 1, 1, 9

[4] P. Auscher, Regularity theorems and heat kernel for elliptic operators, J. London Math. Soc. (2) $\mathbf{5 4}$ (1996), no. 2, 284-296. 6.16

[5] P. Auscher, On necessary and sufficient conditions for $L^{p}$-estimates of Riesz transforms associated to elliptic operators on $\mathbb{R}^{n}$ and related estimates, Mem. Amer. Math. Soc. 186 (2007), no. 871 , xviii+75. $1,2.1,2.1,2.2,2.2,2.2,2.2,2.9,2.11,2.2,6.2,6.2,6.3$

[6] P. Auscher, Change of angle in tent spaces, C. R. Math. Acad. Sci. Paris 349 (2011), no. 5-6, 297-301. 2.2

[7] P. Auscher and A. Axelsson, Weighted maximal regularity estimates and solvability of nonsmooth elliptic systems I, Invent. Math. 184 (2011), no. 1, 47-115. 2.1

[8] P. Auscher, S. Hofmann, M. Lacey, A. McIntosh, and Ph. Tchamitchian, The solution of the Kato square root problem for second order elliptic operators on $\mathbb{R}^{n}$, Ann. of Math. (2) 156 (2002), no. 2, 633-654. 2.1, 2.2

[9] P. Auscher, S. Hofmann, and J.-M. Martell, Vertical versus conical square functions, Trans. Amer. Math. Soc. 364 (2012), no. 10, 5469-5489. 2.9, 6.2, 6.2

[10] P. Auscher, C. Kriegler, S. Monniaux, and P. Portal, Singular integral operators on tent spaces, J. Evol. Equ. 12 (2012), no. 4, 741-765. 1, 2.1, 2.2, 2.2, 2.10

[11] P. Auscher, A. McIntosh, and E. Russ, Hardy spaces of differential forms on Riemannian manifolds, J. Geom. Anal. 18 (2008), no. 1, 192-248. 2.1

[12] P. Auscher and M. Mourgoglou, Representation and uniqueness for boundary value elliptic problems via first order systems, available at http://arxiv.org/pdf/1404.2687v1.pdf, 2014. $2.1,4.2$

[13] F. Bernicot, T. Coulhon, and D. Frey, Gaussian heat kernel bounds through elliptic Moser iteration, available at http://arxiv.org/pdf/1407.3906v2.pdf, 2015. 4.1 
[14] A. Björn and J. Björn, Nonlinear potential theory on metric spaces, EMS Tracts in Mathematics, vol. 17, European Mathematical Society (EMS), Zürich, 2011. 4.1

[15] R. R. Coifman, Y. Meyer, and E. M. Stein, Some new function spaces and their applications to harmonic analysis, J. Funct. Anal. 62 (1985), no. 2, 304-335. 2.1, 7.3, 7.4

[16] R. Dautray and J.-L. Lions, Mathematical analysis and numerical methods for science and technology. Vol. 5, Springer-Verlag, Berlin, 1992, Evolution problems. I, With the collaboration of Michel Artola, Michel Cessenat and Hélène Lanchon, Translated from the French by Alan Craig. 2.1, 3.2, 3.5

[17] L. de Simon, Un'applicazione della teoria degli integrali singolari allo studio delle equazioni differenziali lineari astratte del primo ordine, Rend. Sem. Mat. Univ. Padova 34 (1964), 205223. 2.2

[18] J. Dodziuk, Maximum principle for parabolic inequalities and the heat flow on open manifolds, Indiana Univ. Math. J. 32 (1983), no. 5, 703-716. 1

[19] E. B. Fabes and D. W. Stroock, A new proof of Moser's parabolic Harnack inequality using the old ideas of Nash, Arch. Rational Mech. Anal. 96 (1986), no. 4, 327-338. 7.4

[20] C. Fefferman and E. M. Stein, $H^{p}$ spaces of several variables, Acta Math. 129 (1972), no. 3-4, 137-193. 1, 7.2

[21] S. Hofmann and S. Kim, Gaussian estimates for fundamental solutions to certain parabolic systems, Publ. Mat. 48 (2004), no. 2, 481-496. 1, 3.4, 4.3, 4.3

[22] S. Hofmann and S. Mayboroda, Hardy and BMO spaces associated to divergence form elliptic operators, Math. Ann. 344 (2009), no. 1, 37-116. 2.1, 7, 7.2

[23] Y. Huang, Weighted tent spaces with whitney averages: factorization, interpolation and duality, Math. Zeit. (2015), 1-21, Online. 2.1

[24] T. Hytönen and A. Rosén, On the Carleson duality, Ark. Mat. 51 (2013), no. 2, 293-313. 2.1

[25] T. Iwaniec and C. A. Nolder, Hardy-Littlewood inequality for quasiregular mappings in certain domains in $\mathbf{R}^{n}$, Ann. Acad. Sci. Fenn. Ser. A I Math. 10 (1985), 267-282. 4.1

[26] N. J. Kalton and L. Weis, The $H^{\infty}$-calculus and sums of closed operators, Math. Ann. 321 (2001), no. 2, 319-345. 2.2

[27] C. Kenig and J. Pipher, The Neumann problem for elliptic equations with nonsmooth coefficients, Invent. Math. 113 (1993), no. 3, 447-509. 1, 2.1, 2.1

[28] O. A. Ladyženskaja, V. A. Solonnikov, and N. N. Ural'ceva, Linear and quasilinear equations of parabolic type, Translated from the Russian by S. Smith. Translations of Mathematical Monographs, Vol. 23, American Mathematical Society, Providence, R.I., 1968. 1

[29] P. Li, Uniqueness of $L^{1}$ solutions for the Laplace equation and the heat equation on Riemannian manifolds, J. Differential Geom. 20 (1984), no. 2, 447-457. 1

[30] J.-L. Lions, Sur les problèmes mixtes pour certains systèmes paraboliques dans des ouverts non cylindriques, Ann. Inst. Fourier, Grenoble 7 (1957), 143-182. 1, 1, 1, 2.1, 3.2, 3.3, 3.5

[31] J. Moser, A Harnack inequality for parabolic differential equations, Comm. Pure Appl. Math. 17 (1964), 101-134. 1

[32] J. Nash, Continuity of solutions of parabolic and elliptic equations, Amer. J. Math. 80 (1958), 931-954. 1, 9, 9

[33] L. Nirenberg, On elliptic partial differential equations, Ann. Scuola Norm. Sup. Pisa (3) 13 (1959), 115-162. 4.1

[34] E.M. Ouhabaz, Analysis of heat equations on domains, London Mathematical Society Monographs Series, vol. 31, Princeton University Press, Princeton, NJ, 2005. 2.2 
[35] R.S. Strichartz, Analysis of the Laplacian on the complete Riemannian manifold, J. Funct. Anal. 52 (1983), no. 1, 48-79. 1

[36] J. van Neerven, M. Veraar, and L. Weis, Maximal $\gamma$ regularity, J. Evol. Eq. 15 (2015), 1-42, OnlineFirst. 2.2, 2.9

[37] L. Weis, Operator-valued Fourier multiplier theorems and maximal $L_{p}$-regularity, Math. Ann. 319 (2001), no. 4, 735-758. 2.2 